hep-th/9701043

\title{
Equivalence Between Chain Categories of Representations of Affine $s \ell(2)$ And $N=2$ Superconformal Algebras
}

\author{
B. L. Feigina, A. M. Semikhatov, and I. Yu. Tipunin ${ }^{\mathrm{b}}$ \\ ${ }^{a}$ Landau Institute for Theoretical Physics, Russian Academy of Sciences \\ b Tamm Theory Division, Lebedev Physics Institute, Russian Academy of Sciences
}

Highest-weight type representation theories of the affine $s \ell(2)$ and $N=2$ superconformal algebras are shown to be equivalent modulo the respective spectral flows.

I Introduction 2

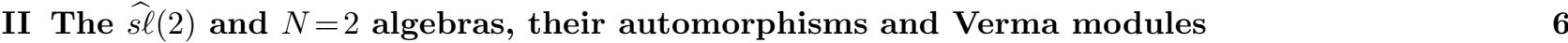

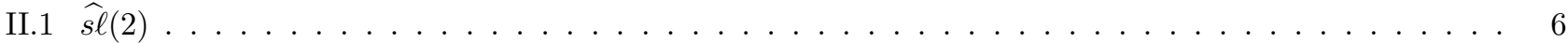

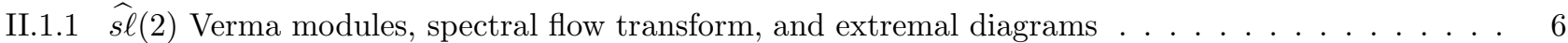

$\mathbb{I I . 1 . 2}$ Singular vectors in $s \ell(2)$ Verma module $\ldots \ldots \ldots \ldots \ldots \ldots \ldots \ldots$

II.1.3 Relaxed Verma modules . . . . . . . . . . . . . . . . . . . . . . . . . . . . . . . . 11

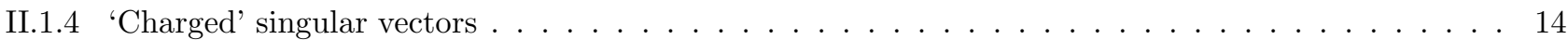

II.1.5 Relation with the previous constructions of some $s \ell(2)$ modules . . . . . . . . . . . . . . . . . . . 14

II.1.6 Singular vectors in relaxed Verma modules $\ldots \ldots \ldots \ldots \ldots \ldots$

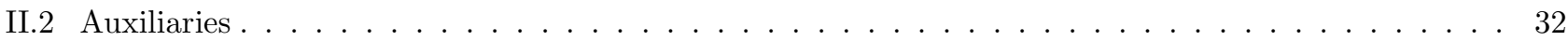

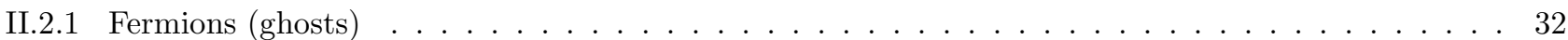

II 2.2 The 'Liouville' scalar . . . . . . . . . . . . . . . . . . . . . . . . . . . . . . . . . . . . . 33

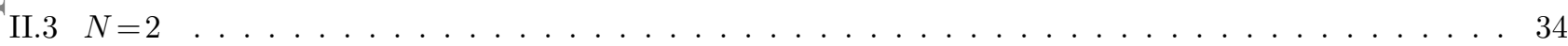

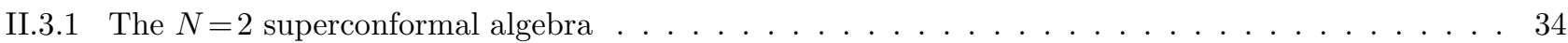

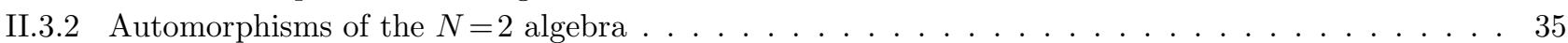

II.3.3 Twisted topological Verma modules $\ldots \ldots \ldots \ldots \ldots \ldots \ldots$. . . . . . . . . . . . . . . 36

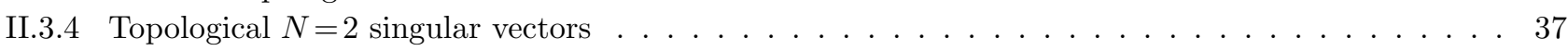

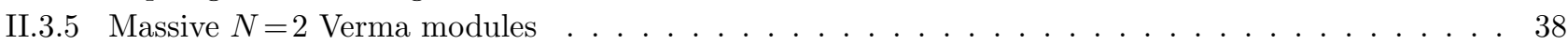

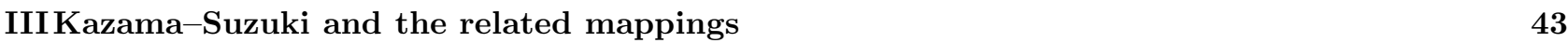

III.1 The Kazama-Suzuki mapping . . . . . . . . . . . . . . . . . . . . . . . . . . . . . . . . 43

III.2 The 'anti'-Kazama-Suzuki mapping . . . . . . . . . . . . . . . . . . . . . . . . . . . . . . . 45

III.3 Composing the KS and anti-KS mappings . . . . . . . . . . . . . . . . . . . . . . . . 46

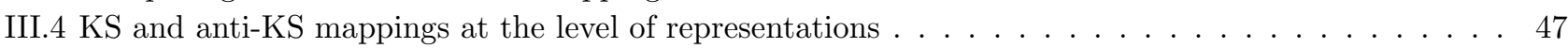

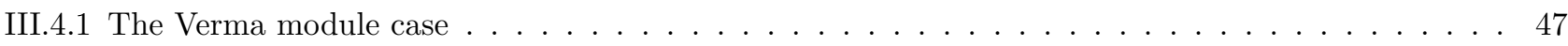

III.4.2 The relaxed $/$ massive Verma module case $\ldots \ldots \ldots \ldots \ldots \ldots$

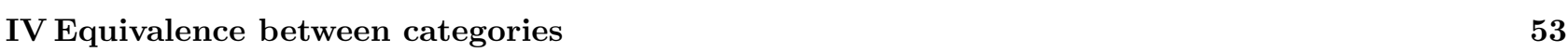

IV.1 Equivalence of Verma chain categories $\ldots \ldots \ldots \ldots \ldots \ldots \ldots \ldots \ldots$

IV.2 Extending the equivalence to highest-weight-type categories $\ldots \ldots \ldots \ldots \ldots \ldots \ldots$

IV.3 Equivalence of the relaxed and massive Verma chain categories . . . . . . . . . . . . . . . . . 56

IV.4 Extending the equivalence to the relaxed highest-weight-type categories $\ldots \ldots \ldots \ldots$. . . . . . . 57

\begin{tabular}{|cc}
\hline V Concluding remarks & 57
\end{tabular} 


\section{Introduction}

In this paper, we study highest-weight type modules over the $N=2$ superconformal algebra and the affine $s \ell(2)$ algebra and demonstrate the equivalence of the two representation theories modulo the respective spectral flows. The motivation comes partly from the previous analysis of $\widehat{s \ell}(2)$ fusion rules [1], which suggests the occurrence of more general modules than the usual Verma modules [2]. The $N=2$ superconformal algebra [3], which is essential in a number of conformal field-theoretic/string-theoretic constructions is known to have various relations to $\widehat{s \ell}(2)$. A prerequisite for constructing complete $N=2$ fusion rules is a better understanding of modules over the $N=2$ algebra, and in particular, in view of a recent progress in constructing $\widehat{s \ell}(2)$ conformal blocks [4, 5, 6], of their relation to $\widehat{s \ell}(2)$ modules.

In what follows, we 'compare' $\widehat{s \ell}(2)$ - and $N=2$ representation theories and then find certain categories built out of representations of these algebras that are equivalent. In particular, we give a complete proof of the claim of [7] that singular vectors in the 'topological' ( $\equiv$ 'chiral') Verma modules over the $N=2$ algebra are isomorphic to singular vectors in $\widehat{s \ell}(2)$ Verma modules. We extend this result along two directions. First, the relation between topological $N=2$ Verma modules and the standard $\widehat{s \ell}(2)$ Verma modules is extended to the equivalence of certain categories of representations of the two algebras, which are essentially the respective twisted $\mathcal{O}$-categories (see [ [ $]$ ]), in fact the categories of chains of such modules related by the respective spectral flows. Hence the importance of twisted (三 spectral-flow-transformed) modules; on the $N=2$ side, such modules have been around for some time [9, 10], while for the $\widehat{s \ell}(2)$ algebra they do not seem to have previously been studied in any detail (see, however, [11]). In general, the spectral flow transformations and the canonical involution constitute the automorphism group of $\widehat{s \ell}(2)$ as well as of the $N=2$ algebra; the action of automorphisms on representations can be defined in a natural way, hence the appearance of twisted modules.

Second, we extend the correspondence between $\widehat{s \ell}(2)$ and $N=2$ Verma modules to representations of a different highest-weight type. For the $N=2$ algebra, one should distinguish between two types of Verma-like objects: the topological Verma modules mentioned above, and the so-called massive $N=2$ Verma modules. The latter are often considered [12] as the 'standard $N=2$ Verma modules'. Their $\widehat{s \ell}(2)$ counterpart, however, is not so standard: these are the relaxed Verma modules, which differ from the usual $\widehat{s \ell}(2)$ Verma modules by somewhat 'relaxed' highest-weight conditions, and as a result possess infinitely many highest-weight vectors. 
On the $\widehat{s \ell}(2)$ side, the standard $\widehat{s \ell}(2)$ Verma modules may appear as submodules of the relaxed Verma modules. On the $N=2$ side, the situation is similar: a massive Verma module may contain submodules that are topological Verma modules (in fact, twisted topological Verma modules; such are, in particular, the submodules generated from the charged $N=2$ singular vectors).

It thus turns out that the modules looking 'standard' on the $N=2$ side correspond to less standard $\widehat{s \ell}(2)$ modules, and vice versa, which is one of the reasons for the appearance of many types of modules we consider in what follows.

A proper identification of relaxed Verma modules may be important for constructing the complete, $s \ell(2 \mid 1)_{q}$-symmetric, fusion rules. The $\widehat{s \ell}(2)$ modules appearing in the fusion constructions and in the constructions of conformal blocks [1, 2, 6, (4) are related to the relaxed Verma modules introduced in this paper. The relaxed Verma modules can be defined as the induced modules from the representations of the Lie algebra $s \ell(2)$ of neither the highest-weight nor the lowest-weight type. In the modules used previously, however, there occur several 'Wakimototype' effects when some singular vectors vanish and cosingular vectors appear instead (this applies to the charged singular vectors).

The main results of this paper are (i) the structural theory of the relaxed $\widehat{s \ell}(2)$ Verma modules, and (ii) the equivalence of categories. As regards the relaxed Verma modules, we classify possible degenerations, explicitly construct singular vectors in the framework of the 'complex-power' approach [13], and describe the nature of the corresponding submodules. The structure of relaxed Verma modules, as regards the appearance of submodules, is identical to that of $N=2$ massive Verma modules; see however 14 for the analysis in intrinsic $N=2$ terms.

As to the equivalence of categories, we establish the pairwise equivalence of the following categories of $\widehat{s \ell}(2)$ modules on the one hand and $N=2$ modules on the other hand (the respective corners of the squares):

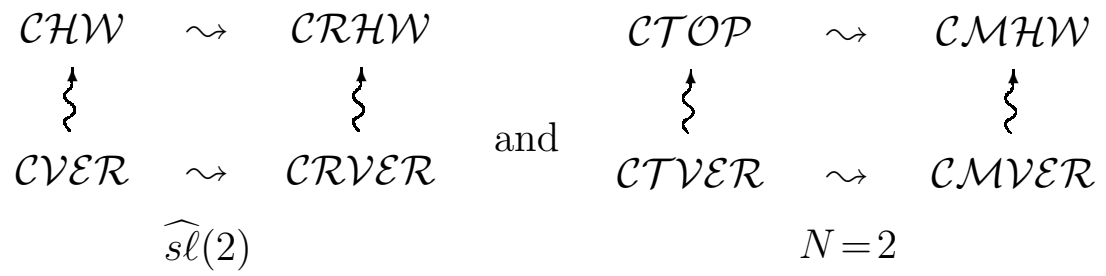

In this diagram, ' $\mathcal{C}$ ' always stands for chains, which we define below, of modules from the corresponding categories, while the categories themselves are as follows. 
In the $\widehat{s \ell}(2)$ square, $\mathcal{V E R}$ consists of the usual $\widehat{s \ell}(2)$ Verma modules together with all their images under the spectral flow (i.e., all the twisted Verma modules, which we also define below). Introducing the spectral-flow-transformed $\widehat{s \ell}(2)$ modules is essential for establishing the relation with $N=2$ modules, where the role of the spectral flow has been appreciated for some time [15, 16]. $\mathcal{R} \mathcal{V E \mathcal { R }}$ consists of (twisted) relaxed Verma modules, which differ from the modules from $\mathcal{V E R}$ by one missing annihilation condition satisfied by the highest-weight vector: as a result, relaxed Verma modules acquire infinitely many 'almost-highest-weight' vectors. Further, in the top line of the $\widehat{s \ell}(2)$ square we have the respective highest-weight-type modules, obtained from the bottom line by taking all possible quotient modules and gluing different modules to each other. These ' $\mathcal{O}$ '-categories include twisted modules as well; in what follows, we give an intrinsic definition of these categories using a criterion which is invariant under twisting. We call $\mathcal{H W}$ and $\mathcal{R H W}$ the categories of (twisted) highest-weight- and relaxed-highest-weight-type modules. For the category $\mathcal{H W}$, therefore, the "universal" role of Verma modules in producing irreducible representations (by taking quotients) is played by the standard $\widehat{s \ell}(2)$ Verma modules $\mathcal{V E R}$. As regards $\mathcal{R H} \mathcal{W}$, on the other hand, distinguished in this way are the relaxed Verma modules $\mathcal{R} \mathcal{V E R}$. As a terminological remark, however, let us note that speaking of Verma modules in the $\widehat{s \ell}(2)$ context, we will always mean only the standard Verma modules $\mathcal{V E R}$, as opposed to the relaxed Verma modules $\mathcal{R} \mathcal{V E R}$.

On the $N=2$ side, $\mathcal{T V E \mathcal { R }}$ are the topological Verma modules and all their spectral flow transforms, while $\mathcal{T O P}$ are the corresponding topological-highest-weight type modules, constructed from modules from $\mathcal{T V E R}$ by taking quotients and gluing. We will also give a spectralflow invariant criterion that characterizes modules from $\mathcal{T O P}$, irrespective of their twists. Further, $\mathcal{M V E R}$ are the twisted 'massive' Verma modules; those with a zero twist are commonly viewed as the 'standard' $N=2$ Verma modules. Finally, $\mathcal{M H W}$ is made up of modules of the same highest-weight type as the twisted massive Verma modules, but contains many more modules, all of which can be constructed out of modules from $\mathcal{M V E R}$ by taking quotients and gluing. These, too, will be defined in 'intrinsic' terms (using the condition of terminating fermionic chains).

The chains of the corresponding modules have to be introduced since the mapping that we use to establish equivalence of categories maps a given $\widehat{s \ell}(2)$ module into a sum involving $N=2$

\footnotetext{
${ }^{1}$ The standard definition of the $\mathcal{O}$ category is less convenient in the case involving twisted modules, because the separation of operators from the algebra into creation and annihilation ones depends on the twist.
} 
modules twisted by all integers. Taking chains of modules makes the respective corners of the two squares in (II.1) equivalent. In application to Verma-like modules, a part of this statement amounts to the isomorphism between singular vectors in the respective modules. As another consequence, one can further derive identities between characters of certain $\widehat{s \ell}(2)$ and $N=2$ modules, as we will do in this paper for the 'Verma' case, and carry over the fusion rules from $\widehat{s \ell}(2)$ to $N=2$ theories (which is left for the future).

The tools essential for the analysis of $\widehat{s \ell}(2)$ and $N=2$ modules include diagrams of extremal vectors. The idea to consider extremal vectors was put forward in [17], where it was observed that a number of representation-theoretic problems can naturally be reformulated, and quite often, solved, in terms of extremal vectors. An independent construction of [10 (and a similar one, [18]) can be considered as a manifestation of this general observation.

As regards singular vectors, we borrow from [10] the construction of $N=2$ singular vectors in terms of continued fermionic operators. We also develop a parallel construction of singular vectors in the relaxed Verma modules over the affine $s \ell(2)$. The latter construction is formulated in quite different terms than the former, however the two constructions turn out to be equivalent and, moreover, allow direct mappings between each other. An important point is that the isomorphism between singular vectors requires that one consider the $N=2$ singular vectors that satisfy twisted highest-weight conditions. Allowing the singular vectors to satisfy twisted highest-weight conditions makes it possible to choose those representatives among singular vectors with different twists that generate maximal submodules (see [14] as to how this 'eliminates' the superfluous $N=2$ subsingular vectors). An essential point that we emphasize is the necessity to consider the entire extremal diagrams, rather than their fixed representatives, in order to correctly describe the structure of submodules.

In Section [1, we introduce the $\widehat{s \ell}(2)$ and $N=2$ superconformal algebras, review some of their properties (in particular, the corresponding spectral flows), investigate the structure of the corresponding Verma modules, and define different categories of such modules. We also introduce auxiliary free-field systems. In Section III, we recall the simplest Kazama-Suzuki (KS) mapping [19, 20 and construct another mapping, which is essentially inverse to KS even though it acts between somewhat different spaces; it will be called the anti-KS mapping. These are used in Section $\square$ to prove equivalence between the respective categories of $\widehat{s \ell}(2)$ and $N=2$ representations. 
The paper is rather long, which may be partly excused by a large number of modules that we have to introduce in order to keep balance between the $\widehat{s \ell}(2)$ and $N=2$ sides. We omit some of the proofs.

\section{The $\widehat{s \ell}(2)$ and $N=2$ algebras, their automorphisms and Verma modules}

In this section, we introduce the $\widehat{s \ell}(2)$ and the $N=2$ superconformal algebras. We recall their properties, define several types of modules and give theorems describing the structure of singular vectors in these modules. We also introduce free-field theories that will be needed for a constructive realization of the mapping between representations of these two algebras.

\section{II.1 $\widehat{s \ell}(2)$}

Some of the facts given below about the affine $s \ell(2)$ algebra are well known, yet we hope to avoid being too boring. However, we try to give more details about twisted and 'relaxed' modules. In section [1.1.6, we describe singular vectors in relaxed Verma modules, where the situation is more involved than with the standard Verma modules, and give structural theorems about this class of $\widehat{s \ell}(2)$ modules.

\section{II.1.1 $\widehat{s \ell}(2)$ Verma modules, spectral flow transform, and extremal diagrams}

The level- $k$ algebra is defined as

$$
\begin{aligned}
& {\left[J_{m}^{0}, J_{n}^{ \pm}\right]= \pm J_{m+n}^{ \pm}, \quad\left[J_{m}^{0}, J_{n}^{0}\right]=\frac{K}{2} m \delta_{m+n, 0}} \\
& {\left[J_{m}^{+}, J_{n}^{-}\right]=K m \delta_{m+n, 0}+2 J_{m+n}^{0}}
\end{aligned}
$$

(where $K$ is the central element, which we will not distinguish from its value $k$ in representations). Commutation relations (II.1) correspond to the following operator product expansions of currents:

$$
\begin{aligned}
J^{0}(z) J^{ \pm}(w) & = \pm \frac{J^{ \pm}}{z-w}, \quad J^{0}(z) J^{0}(w)=\frac{k / 2}{(z-w)^{2}} \\
J^{+}(z) J^{-}(w) & =\frac{k}{(z-w)^{2}}+\frac{2 J^{0}}{z-w} .
\end{aligned}
$$

In terms of the currents, the Sugawara energy-momentum tensor has the form

$$
T^{\text {Sug }}=\frac{1}{k+2}\left(J^{0} J^{0}+\frac{1}{2}\left(J^{+} J^{-}+J^{-} J^{+}\right)\right) .
$$


Here and in what follows, we assume $k \neq-2$. The usual mode expansion of the energymomentum tensors is $T^{\mathrm{Sug}}(z)=\sum_{n \in \mathbb{Z}} L_{n}^{\mathrm{Sug}} z^{-n-2}$, and similarly for the other energy-momentum tensors that we work with below.

We now define the Verma module $\mathcal{M}_{j, k}$ with the highest-weight vector $|j, k\rangle_{s \ell(2)}$ in the standard manner, by imposing the following annihilation conditions on the highest-weight vector:

$$
\begin{aligned}
& J_{\geq 0}^{+}|j, k\rangle_{s \ell(2)}=J_{\geq 1}^{0}|j, k\rangle_{s \ell(2)}=J_{\geq 1}^{-}|j, k\rangle_{s \ell(2)}=0, \\
& J_{0}^{0}|j, k\rangle_{s \ell(2)}=j|j, k\rangle_{s \ell(2)}, \quad K|j, k\rangle_{s \ell(2)}=k|j, k\rangle_{s \ell(2)}, \quad j, k \in \mathbb{C} .
\end{aligned}
$$

The module is freely generated from $|j, k\rangle_{s \ell(2)}$ by the remaining modes of $J^{+}, J^{-}$, and $J^{0}$; thus the character of $\mathcal{M}_{j, k}$, is given by

$$
\chi_{j, k}^{s \ell(2)}(z, q)=\operatorname{Tr}_{\mathcal{M}_{j, k}}\left(q^{L_{0}^{\mathrm{sug}}} z^{J_{0}^{0}}\right)=\frac{z^{j} q^{\Delta_{j}}}{\prod_{i=1}^{\infty}\left(1-z q^{i}\right) \prod_{i=0}^{\infty}\left(1-z^{-1} q^{i}\right) \prod_{i=1}^{\infty}\left(1-q^{i}\right)},
$$

where $\Delta_{j}=j(j+1) /(k+2)$ is the Sugawara dimension of the highest-weight state.

The $\widehat{s \ell}(2)$ Verma modules can conveniently be described with the help of the extremal diagram

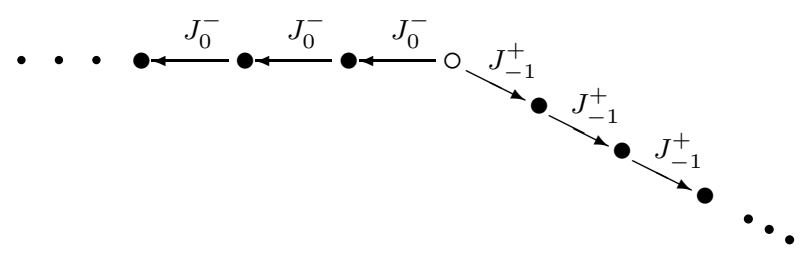

which expresses the fact that $J_{-1}^{+}$and $J_{0}^{-}$are the highest-level operators that do not yet annihilate the highest-weight state $\circ$. All the other states of the module, according to their (charge, level) bigrading, lie in the interior of the angle in the diagram. In these conventions, e.g., $J_{-1}^{0}$ is represented by a downward vertical arrow.

We will need automorphisms of the affine $s \ell(2)$ algebra. These are the canonical involution and the spectral flow transform [11]

$$
\mathcal{U}_{\theta}: \quad J_{n}^{+} \mapsto J_{n+\theta}^{+}, \quad J_{n}^{-} \mapsto J_{n-\theta}^{-}, \quad J_{n}^{0} \mapsto J_{n}^{0}+\frac{k}{2} \theta \delta_{n, 0}, \quad \theta \in \mathbb{Z} .
$$

Spectral flow transformations with $\theta \in 2 \mathbb{Z}$ are the continuation to the entire algebra of the affine Weyl group action. In general, $\widehat{s \ell}(2)$-modules are not invariant under the spectral flow and are mapped into 'twisted' modules.

\footnotetext{
${ }^{2} \mathrm{~A}$ notable exception is provided, for $k \in \mathbb{N}$, by the integrable representations. These are invariant under the action of spectral flow transformations with $\theta \in 2 \mathbb{Z}$, however the spectral flow transformations with $\theta \in 2 \mathbb{Z}+1$ map one integrable representation into another.
} 
Definition II.1 A twisted Verma module $\mathfrak{M}_{j, k, \theta}$ is freely generated by $J_{\leq \theta-1}^{+}, J_{\leq-\theta}^{-}$, and $J_{\leq-1}^{0}$ from a twisted highest-weight vector $|j, k ; \theta\rangle_{s \ell(2)}$ defined by the conditions

$$
\begin{aligned}
& J_{\geq \theta}^{+}|j, k ; \theta\rangle_{s \ell(2)}=J_{\geq 1}^{0}|j, k ; \theta\rangle_{s \ell(2)}=J_{\geq-\theta+1}^{-}|j, k ; \theta\rangle_{s \ell(2)}=0, \\
& \left(J_{0}^{0}+\frac{k}{2} \theta\right)|j, k ; \theta\rangle_{s \ell(2)}=j|j, k ; \theta\rangle_{s \ell(2)} .
\end{aligned}
$$

The respective extremal diagrams are obvious 'rotations' of (II.6). We identify $|j, k\rangle_{s \ell(2)}=$ $|j, k ; 0\rangle_{s \ell(2)}$.

All possible (integral) twists of Verma modules constitute the category $\mathcal{V E R}$. This category is very 'small': already in the untwisted case, many (if not all) interesting representations are not Verma modules, but rather can be obtained from Verma modules by taking quotients and 'gluing'. This gives the category $\mathcal{O}$ (see [8]), in which every irreducible representation is a quotient of a Verma module. An important feature, which in fact characterizes the modules from category $\mathcal{O}$ is that the action of all the annihilation operators on any vector from a given module spans out a finite-dimensional space (and the modules must be finite-generated). In terms of extremal diagrams, this means simply that the annihilators act into the interior of the angle

$$
\underset{J_{1}^{-}}{\stackrel{\cdots}{*} \cdot \frac{\cdot}{J_{0}^{+}}} \cdot
$$

and therefore continuing to act with the annihilators on a state from a module from $\mathcal{O}$, one inevitably reaches the 'edge', at which the annihilators act by zero.

We need an $\mathcal{O}$-type category that would include the twisted modules. A remarkable fact is that it does not have to be defined by 'enumerating' different twistings of $\mathcal{O}$, as

$$
\bigcup_{\theta \in \mathbb{Z}} \mathcal{O}_{\theta}=\bigcup_{\theta \in \mathbb{Z}} \mathcal{U}_{\theta} \mathcal{O}
$$

instead, there exists an intrinsic definition. Consider first

Definition II.2 Let $|X\rangle$ be an element of a module over the affine sl(2) algebra and let us fix an integer $\theta$. For $J$ being either $J^{+}$or $J^{-}$, we say that the $J_{\theta}$-chain terminates on $|X\rangle$, and write $\left(J_{\theta}\right)^{+\infty}|X\rangle=0$ if

$$
\exists N \in \mathbb{Z}, \quad n \geq N:\left(J_{\theta}\right)^{n}|X\rangle=0 .
$$

Now, the property discussed before Eq. (III.9) is invariant under the twists. This underlies the next definition. Before giving it, let us assume once and for all that all the modules under 
consideration are finite-generated (this requirement is often imposed in the form of the condition that the modules be graded with respect to an appropriate Virasoro generator $\left.L_{0}\right)$. Then,

Definition II.3 Objects of the category $\mathcal{H W}$ of $\widehat{s \ell}(2)$ twisted highest-weight-type representations are modules $\mathfrak{U}$ over the affine $s \ell(2)$ algebra such that the following conditions are satisfied:

1. $J_{0}^{0}$ is a diagonalizable operator, i.e. $\mathfrak{U}$ can be decomposed into a direct sum of $J_{0}^{0}$ eigenspaces;

2. The action with all the operators $J_{p}^{0}, p \in \mathbb{N}$, on any element $|X\rangle \in \mathfrak{U}$ spans a finitedimensional space;

3. for any element $|X\rangle$ of $\mathfrak{U}$,

$$
\begin{array}{lll}
\forall n \in \mathbb{Z} & \text { either } & \left(J_{n}^{+}\right)^{+\infty}|X\rangle=0 \\
& \text { or } & \left(J_{-n}^{-}\right)^{+\infty}|X\rangle=0
\end{array}
$$

Morphisms are standard homomorphisms between $\widehat{s \ell}(2)$-modules.

Thus conditions (ㅍ.10) do not distinguish between different $\theta$-twists of category $\mathcal{O}$. Heuristically, these conditions say that each straight line (in the conventions of (II.6)) of $\widehat{s \ell}(2)$ operators acting in a given module from the category intersects the boundary of the module, or simply that the extremal diagram, even though not of the simple form (II.6), is nevertheless angle-shaped, the angle being strictly less than 180 degrees.

\section{II.1.2 Singular vectors in $\widehat{s \ell}(2)$ Verma modules}

A singular vector in a Verma module $\mathcal{M}_{j, k}$ is a vector that is not proportional to the highest-weight vector and nevertheless satisfies the same annihilation conditions (II.4) as the highest-weight vector does.

For $\alpha \in \mathbb{C}$, we introduce the objects $\left(J_{-1}^{+}\right)^{\alpha}$ and $\left(J_{0}^{-}\right)^{\alpha}$ that implement the standard action of generators of the affine Weyl group on the space of highest-weights, see [13] for the details. These objects correspond to reflections with respect to two positive simple roots of $\widehat{s \ell}(2)$. In fact $\left(J_{-1}^{+}\right)^{\alpha}$ and $\left(J_{0}^{-}\right)^{\alpha}$ define the following Weyl group action on the line $k=$ const in the $k j$ plane of highest weights:

$$
\begin{aligned}
& \left(J_{0}^{-}\right)^{2 j+1}:|j, k\rangle_{s \ell(2)} \rightarrow|-1-j, k\rangle_{s \ell(2)} \\
& \left(J_{-1}^{+}\right)^{k+1-2 j}:|j, k\rangle_{s \ell(2)} \rightarrow|k+1-j, k\rangle_{s \ell(2)}
\end{aligned}
$$


The action of $\left(J_{-1}^{+}\right)^{\alpha}$ and $\left(J_{0}^{-}\right)^{\alpha}$ can be extended from the highest-weight vectors to the Verma modules over these vectors. The extension is given by the following formulas:

$$
\begin{aligned}
\left(J_{0}^{-}\right)^{\alpha} J_{m}^{+} & =-\alpha(\alpha-1) J_{m}^{-}\left(J_{0}^{-}\right)^{\alpha-2}-2 \alpha J_{m}^{0}\left(J_{0}^{-}\right)^{\alpha-1}+J_{m}^{+}\left(J_{0}^{-}\right)^{\alpha}, \\
\left(J_{0}^{-}\right)^{\alpha} J_{m}^{0} & =\alpha J_{m}^{-}\left(J_{0}^{-}\right)^{\alpha-1}+J_{m}^{0}\left(J_{0}^{-}\right)^{\alpha}, \\
\left(J_{-1}^{+}\right)^{\alpha} J_{m}^{-} & =-\alpha(\alpha-1) J_{m-2}^{+}\left(J_{-1}^{+}\right)^{\alpha-2}-k \alpha \delta_{m-1,0}\left(J_{-1}^{+}\right)^{\alpha-1}+2 \alpha J_{m-1}^{0}\left(J_{-1}^{+}\right)^{\alpha-1}+J_{m}^{-}\left(J_{-1}^{+}\right)^{\alpha}, \\
\left(J_{-1}^{+}\right)^{\alpha} J_{m}^{0} & =-\alpha J_{m-1}^{+}\left(J_{-1}^{+}\right)^{\alpha-1}+J_{m}^{0}\left(J_{-1}^{+}\right)^{\alpha},
\end{aligned}
$$

which can be derived for $\alpha$ being a positive integer and then postulated for an arbitrary complex $\alpha$. Then,

\section{Theorem II.4}

I. ([21]) A singular vector exists in the Verma module $\mathcal{M}_{j, k}$ over the affine sl(2) algebra if and only if $j=\mathrm{j}^{+}(r, s, k)$ or $j=\mathrm{j}^{-}(r, s, k)$, where

$$
\left.\begin{array}{l}
\mathrm{j}^{+}(r, s, k)=\frac{r-1}{2}-(k+2) \frac{s-1}{2} \\
\mathrm{j}^{-}(r, s, k)=-\frac{r+1}{2}+(k+2) \frac{s}{2}
\end{array}\right\} \quad \begin{aligned}
& r, s \in \mathbb{N}, \\
& k \in \mathbb{C} .
\end{aligned}
$$

II. ([13]) All singular vectors in the module $\mathcal{M}_{\mathbf{j}^{ \pm}(r, s, k), k}$ are given by the explicit constructions:

$$
\begin{aligned}
\left|\mathrm{MFF}^{+}(r, s, k)\right\rangle=\left(J_{0}^{-}\right)^{r+(s-1)(k+2)}\left(J_{-1}^{+}\right)^{r+(s-2)(k+2)}\left(J_{0}^{-}\right)^{r+(s-3)(k+2)} \ldots \\
\cdot\left(J_{-1}^{+}\right)^{r-(s-2)(k+2)}\left(J_{0}^{-}\right)^{r-(s-1)(k+2)}\left|\mathrm{j}^{+}(r, s, k), k\right\rangle_{s \ell(2)}, \\
\left|\mathrm{MFF}^{-}(r, s, k)\right\rangle=\left(J_{-1}^{+}\right)^{r+(s-1)(k+2)}\left(J_{0}^{-}\right)^{r+(s-2)(k+2)}\left(J_{-1}^{+}\right)^{r+(s-3)(k+2)} \ldots \\
\cdot\left(J_{0}^{-}\right)^{r-(s-2)(k+2)}\left(J_{-1}^{+}\right)^{r-(s-1)(k+2)}\left|\mathrm{j}^{-}(r, s, k), k\right\rangle_{s \ell(2)} .
\end{aligned}
$$

Remark II.5 As is well known [13], the factors in ([1.14) correspond to the affine Weyl group reflections with respect to the simple roots. When there are several singular vectors in a given Verma module, one can have different solutions to equations ([I.13) for $(r, s)$, each such pair yielding a singular vector as given by Eqs. ([1.14). Whenever we say "all singular vectors", we mean, in accordance with [22], that the singular vectors are constructed either directly as in (II.14) or - which may be the case for rational $k$-by composing the factors from two such formulas (the latter being equivalent to truncating a formula from (II.14) as soon as the corresponding Weyl reflections produce a highest weight that differs from the original one by a negative integral multiple of a positive root).

Clearly, singular vectors in the twisted modules $\mathfrak{M}_{j, k ; \theta}$ follow from the standard singular vectors ([1.14) by applying the spectral flow transform. 
Equivalence between $\widehat{s \ell}(2)$ and $N=2$ representations

\section{II.1.3 Relaxed Verma modules}

A different class of $\widehat{s \ell}(2)$ modules can be introduced by relaxing annihilation conditions (II.8):

Definition II.6 For $\theta \in \mathbb{Z}$, the relaxed twisted Verma module $\mathfrak{R}_{j, \Lambda, k ; \theta}$ is generated from the state $|j, \Lambda, k ; \theta\rangle_{s \ell(2)}$ that satisfies the annihilation conditions

$$
J_{\geq \theta+1}^{+}|j, \Lambda, k ; \theta\rangle_{s \ell(2)}=J_{\geq 1}^{0}|j, \Lambda, k ; \theta\rangle_{s \ell(2)}=J_{\geq-\theta+1}^{-}|j, \Lambda, k ; \theta\rangle_{s \ell(2)}=0
$$

by a free action of the operators $J_{\leq \theta-1}^{+}, J_{\leq-\theta-1}^{-}$, and $J_{\leq-1}^{0}$ and by the action of operators $J_{\theta}^{+}$ and $J_{-\theta}^{-}$subject to the constraint

$$
J_{-\theta}^{-} J_{\theta}^{+}|j, \Lambda, k ; \theta\rangle_{s \ell(2)}=\Lambda|j, \Lambda, k ; \theta\rangle_{s \ell(2)} .
$$

In addition, the highest-weight state $|j, \Lambda, k ; \theta\rangle_{\text {sl(2) }}$ satisfies

$$
\left(J_{0}^{0}+\frac{k}{2} \theta\right)|j, \Lambda, k ; \theta\rangle_{s \ell(2)}=j|j, \Lambda, k ; \theta\rangle_{s \ell(2)} .
$$

The corresponding extremal diagram opens up to the straight angle; in the case of $\theta=0$ it thus becomes

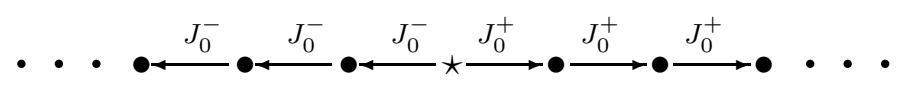

The state marked with $\star$ is the above $|j, \Lambda, k ; \theta\rangle_{s \ell(2)}$, characterized, besides the level, by two quantum numbers $j$ and $\Lambda$. The other states $|j, \Lambda, k ; \theta| n\rangle_{s \ell(2)}, n \in \mathbb{Z}$, in the top floor are

$$
|j, \Lambda, k ; \theta| n\rangle_{s \ell(2)}= \begin{cases}\left(J_{-\theta}^{-}\right)^{-n}|j, \Lambda, k ; \theta\rangle_{s \ell(2)}, & n<0 \\ \left(J_{\theta}^{+}\right)^{n}|j, \Lambda, k ; \theta\rangle_{s \ell(2)}, & n>0\end{cases}
$$

with $|j, \Lambda, k ; \theta| 0\rangle_{s \ell(2)}=|j, \Lambda, k ; \theta\rangle_{s \ell(2)}$. We also set $\left.\left.|j, \Lambda, k| n\right\rangle_{s \ell(2)}=|j, \Lambda, k ; 0| n\right\rangle_{s \ell(2)}$. The Sugawara dimension of $|j, \Lambda, k ; \theta| 0\rangle_{s \ell(2)}$ is

$$
\Delta=\frac{j^{2}+j+\Lambda}{k+2}-\theta j+\frac{k}{4} \theta^{2}
$$

Remark II.7 As we have already remarked, speaking of Verma modules in the $\widehat{s \ell}(2)$ context, we will always mean only the standard Verma modules, as opposed to the relaxed Verma modules; the same will apply to Verma highest-weight conditions, by which we will mean (possibly spectral-flow-transformed) highest-weight conditions in $\mathfrak{M}_{j, k ; \theta}$, rather than Eqs. (II.15). 
By the relative charge of a state $|v\rangle \in \mathcal{R}_{j, \Lambda, k}$ we understand $x=y-j$, where $J_{0}^{0}|v\rangle=y|v\rangle$ (and $J_{0}^{0}|j, \Lambda, k\rangle=j|j, \Lambda, k\rangle$ ). Thus, states with a negative (positive) relative charge are on the left (resp., on the right) of $\star=|j, \Lambda, k\rangle$.

In the generic case, one can travel both ways along the extremal diagram: when $\theta=0$, for example, 'untwisted' diagram ([I.18) acquires a 'fat' form

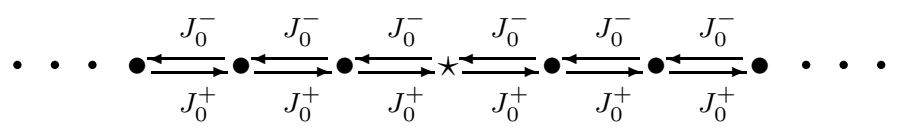

while the one with $\theta=-1$,

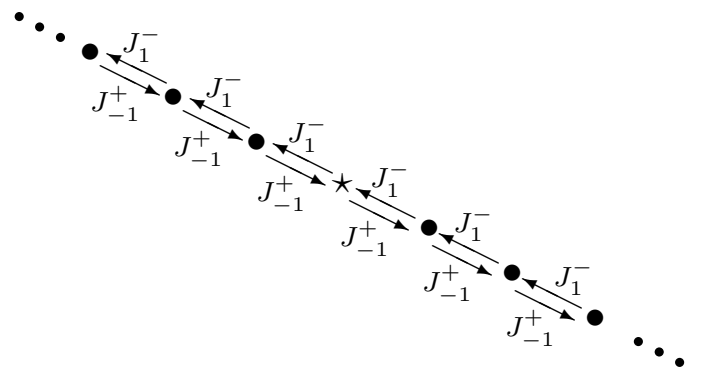

etc. Thus all the points are equivalent $(\star \leadsto \bullet)$, since the composition of the direct and the inverse arrows does in each case result only in a factor. However, this factor may vanish for some values of the parameters, which gives rise to the ordinary Verma modules. Namely, in the untwisted case (【I.18), we have

$$
\begin{aligned}
\left.J_{0}^{-}|j, \Lambda, k| n\right\rangle_{s \ell(2)} & =|j, \Lambda, k| n-1\rangle_{s \ell(2)} \\
\left.J_{0}^{+}|j, \Lambda, k| n-1\right\rangle_{s \ell(2)} & =(\Lambda-n(n-1)-2(n-1) j)|j, \Lambda, k| n\rangle_{s \ell(2)}, \\
\left.n \geq 0: \quad J_{0}^{+}|j, \Lambda, k| n\right\rangle_{s \ell(2)} & =|j, \Lambda, k| n+1\rangle_{s \ell(2)} \\
\left.J_{0}^{-}|j, \Lambda, k| n+1\right\rangle_{s \ell(2)} & =(\Lambda-n(n+1)-2 n j)|j, \Lambda, k| n\rangle_{s \ell(2)}
\end{aligned}
$$

and similarly for non-zero twists $\theta \neq 0$. Whenever the parameters are such that, e.g., $J_{0}^{+} \approx 0$ at a certain step, one cannot come back to the $\star$ state by acting with $J_{0}^{+}$:

$$
\left.n \leq-1: \quad \Lambda=n(n+1)+2 n j \quad \Longrightarrow \quad J_{0}^{+}|j, \Lambda, k| n\right\rangle_{s \ell(2)}=0 .
$$

One keeps on acting with $J_{-1}^{+}$instead (one mode down), and thus the extremal diagram branches as

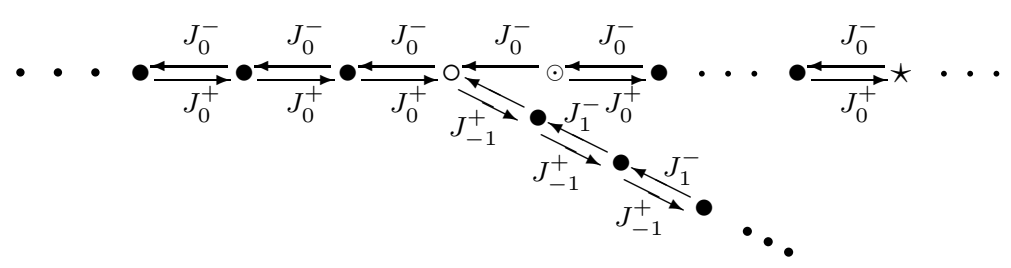


Therefore, any Verma module can be thought of as a submodule of a relaxed Verma module. Taking the quotient with respect to this Verma submodule (or, in the notation of (II.25), with respect to $\circ$ ), we see that the state $\odot$ would satisfy Verma highest-weight conditions (II.8) with $\theta=1$. Hence, each Verma module is at the same time a quotient module of the appropriately twisted relaxed Verma module.

The situation is similar when $J_{0}^{-} \approx 0$ at a certain stage in diagram (II.21):

$$
\left.n \geq 1: \quad \Lambda=n(n-1)+2(n-1) j \quad \Longrightarrow \quad J_{0}^{-}|j, \Lambda, k| n\right\rangle_{s \ell(2)}=0 .
$$

Then the branching of the extremal diagram is a mirror image of ([I.25), and the submodule is given by the spectral flow transform with $\theta=1$ of a standard Verma module. In both cases ([1.24) and ([1.26), we have

$$
\left.\left.J_{0}^{0}|j, \Lambda, k| n\right\rangle_{s \ell(2)}=(j+n)|j, \Lambda, k| n\right\rangle_{s \ell(2)}, \quad n \in \mathbb{Z} .
$$

Hence the Verma-module spin is $j_{\text {Verma }}=j+n$ for $n<0$ (where the Verma submodule in the relaxed module is untwisted), and $j_{\text {Verma }}=j+n+\frac{k}{2}$ for $n>0$ (where the Verma module is twisted by $\theta=1)$.

By a straightforward application of the spectral flow, the above considerations translate into those for the twisted relaxed Verma modules; diagram ([I.25) changes accordingly.

As in the Verma case, we now extend the category $\mathcal{R} \mathcal{V E R}$ of all twisted relaxed Verma modules to a larger category $\mathcal{R H W}$ of arbitrary (twisted) relaxed-highest-weight-type modules. We use the same method as in the case of the $\mathcal{H W}$ category. Now that the angle in the extremal diagrams has opened up to the straight angle, the extremal diagrams certainly contains straight lines that are infinite on both sides. However, a condition on the class of modules can still be given in the form of the requirement that, starting with any vector from a given module, the action with any of the bent $J^{ \pm}$-chains

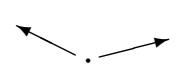

terminates:

Definition II.8 The objects of category $\mathcal{R H \mathcal { W }}$ are $\widehat{\operatorname{s\ell }}(2)$ modules $\mathfrak{U}$ such that the following conditions are satisfied:

1. $J_{0}^{0}$ is a diagonalizable operator; 
2. The action with all $J_{p}^{0}, p \in \mathbb{N}$, on any element $|X\rangle \in \mathfrak{U}$ spans a finite-dimensional space;

3. for any element $|X\rangle$ of $\mathfrak{U}$,

$$
\begin{array}{lll}
\forall \theta \in \mathbb{Z} & \text { either } & \left(J_{\theta}^{+}\right)^{+\infty}|X\rangle=0 \\
& \text { or } & \left(J_{-\theta+1}^{-}\right)^{+\infty}|X\rangle=0
\end{array}
$$

Morphisms are standard homomorphisms between $\widehat{s \ell}(2)$-modules.

Again, this condition is invariant under the spectral flow, and thus selects all the twisted modules, none of which can be 'overrelaxed' in the sense of its extremal diagram occupying more than half a plane.

\section{II.1.4 'Charged' singular vectors}

The 'Verma points' encountered in the top floor of an extremal diagram can of course be viewed as singular vectors in the corresponding relaxed Verma module. The construction of these singular vectors is now obvious: we define

$$
\Lambda_{\mathrm{ch}}(p, j)=p(p+1)+2 p j, \quad p \in \mathbb{Z},
$$

then the state

$$
|C(p, j, k)\rangle_{s \ell(2)}= \begin{cases}\left(J_{0}^{-}\right)^{-p}\left|j, \Lambda_{\mathrm{ch}}(p, j), k\right\rangle_{s \ell(2)}, & p \leq-1 \\ \left(J_{0}^{+}\right)^{p+1}\left|j, \Lambda_{\mathrm{ch}}(p, j), k\right\rangle_{s \ell(2)}, & p \geq 0\end{cases}
$$

satisfies the Verma highest-weight conditions for $p \leq-1$ and the twisted Verma highest-weight conditions with the twist parameter $\theta=1$ for $p \geq 1$. We would like to stress that (II.30) follows immediately from the analysis of extremal diagrams. In fact, these singular vectors may not even deserve a special name, and we mention them specifically in order to make the presentation parallel to the one for the $N=2$ algebra, where 'charged' singular vectors are traditionally considered as a separate notion [12] (although they are given by an equivalently simple construction [10], see ([1.126) ).

\section{II.1.5 Relation with the previous constructions of some $\widehat{s \ell}(2)$ modules}

It is of some interest to see how the $\widehat{s \ell}(2)$ modules constructed using the 'functional realization' of $s \ell(2)$, which have appeared in the study of fusion, are related to relaxed Verma 
modules introduced in this paper. In the untwisted case, consider top-level states (II.18) and assume that $\Lambda$ is expressed through a superficial variable $\mu$ as

$$
\Lambda=\mu(\mu-1-2 j)
$$

(given $\Lambda$ and $j$, we would in general have two different values of $\mu$, but we do not discuss now how they can be distinguished). Then, assuming all the relevant factors non-vanishing, renormalize states ([I.19) into

$$
|j, \Lambda(\mu, j), k| n\rangle_{s \ell(2)}= \begin{cases}\left.f_{-}(n, \mu)|j, \Lambda(\mu, j), k| n\right\rangle^{*}, & n \leq-1 \\ |j, \Lambda(\mu, j), k| 0\rangle^{*}, & n=0 \\ \left.f_{+}(n, j, \mu)|j, \Lambda(\mu, j), k| n\right\rangle^{*}, & n \geq 1\end{cases}
$$

where

$$
\begin{aligned}
f_{-}(n, \mu) & =(-1)^{n} \frac{\Gamma(1-\mu-n)}{\Gamma(1-\mu)}, & & n \leq-1 \\
f_{+}(n, j, \mu) & =(-1)^{n} \frac{\Gamma(2 j+1-\mu+n)}{\Gamma(2 j+1-\mu)}, & & n \geq 1
\end{aligned}
$$

Now, we parametrize $\mu$ and $j$ as

$$
\begin{aligned}
j & =\widetilde{\mu}, \\
\mu & =\widetilde{\mu}-s,
\end{aligned}
$$

and introduce a simplified notation

$$
\left.|s, \widetilde{\mu}, k, n\rangle^{\#} \equiv|\widetilde{\mu},(s-\widetilde{\mu})(s+\widetilde{\mu}+1), k| n\right\rangle^{*} .
$$

Then Eqs. (【I.23) rewrite as

$$
\begin{aligned}
J_{0}^{+}|s, \widetilde{\mu}, k, n\rangle^{\#} & =-(\widetilde{\mu}+n+s+1)|s, \widetilde{\mu}, k, n+1\rangle^{\#}, \\
J_{0}^{0}|s, \tilde{\mu}, k, n\rangle^{\#} & =(\widetilde{\mu}+n)|s, \widetilde{\mu}, k, n\rangle^{\#}, \\
J_{0}^{-}|s, \widetilde{\mu}, k, n\rangle^{\#} & =(\widetilde{\mu}+n-s-1)|s, \widetilde{\mu}, k, n-1\rangle^{\#} .
\end{aligned}
$$

We can consider the set of states $|s, \widetilde{\mu}, k, \cdot\rangle^{\#}$ as representing a state

$$
\| s, \tilde{\mu}, k\rangle\rangle^{\#}=\left\{|s, \tilde{\mu}, k, \cdot\rangle^{\#}\right\}
$$

which can be written in the ' $x$-representation' as

$$
\langle x \| s, \widetilde{\mu}, k\rangle\rangle^{\#}=\sum_{n \in \mathbb{Z}}(-1)^{n} x^{\tilde{\mu}+s+n}|s, \widetilde{\mu}, k, n\rangle^{\#},
$$


so that the action of $J_{0}^{0, \pm}$ is represented by the right action of the generators

$$
\begin{aligned}
S^{+} & =\frac{\partial}{\partial x}, \\
S^{0} & =x \frac{\partial}{\partial x}-s, \\
S^{-} & =-x^{2} \frac{\partial}{\partial x}+2 s x .
\end{aligned}
$$

Denote by $P_{s, \widetilde{\mu}, k}$ the module freely generated from the states $|s, \widetilde{\mu}, k, n\rangle^{\#}, n \in \mathbb{Z}$, by $J_{\leq-1}^{ \pm, 0}$.

We see from ([I.24) and (【I.26) that a Verma highest-weight state (a charged singular vector) occurs in the extremal diagram whenever

$$
\mu=2 j+p, \quad p \in \mathbb{Z}, \quad \text { or } \quad \mu=p, \quad p \in \mathbb{Z} .
$$

Precisely at these values of $\mu$, one of the functions $f_{+}$or $f_{-}$either has a pole or is identically zero, which invalidates relations ([I.31). Then the charged singular vectors disappear and cosingular vectors appear instead.

\section{II.1.6 Singular vectors in relaxed Verma modules}

We are going to describe singular vectors in relaxed Verma modules only in the 'untwisted' case $\theta=0$; similar results for modules with $\theta \neq 0$ can be obtained immediately by applying the spectral flow transform.

Our strategy is to reduce the relaxed case to the case of ordinary Verma modules. Given a relaxed Verma module $\mathcal{R}_{j, \Lambda, k}$, we can arrive at an ordinary Verma module by the following trick. The states $\left(J_{0}^{-}\right)^{-\mu}|j, \Lambda, k\rangle$ and $\left(J_{0}^{+}\right)^{\mu+1}|j, \Lambda, k\rangle$ satisfy Verma highest-weight conditions (II.8) with $\theta=0$ or 1 respectively whenever $\mu$ satisfies

$$
\mu^{2}+(2 j+1) \mu-\Lambda=0
$$

Therefore, the states

$$
\begin{aligned}
& \left|j_{-}, k ; 0\right\rangle_{s \ell(2)}=\left(J_{0}^{-}\right)^{-\mu}|j, \Lambda, k\rangle, \quad\left|j_{-}^{\prime}, k ; 0\right\rangle_{s \ell(2)}=\left(J_{0}^{-}\right)^{-\mu^{\prime}}|j, \Lambda, k\rangle, \quad \mu^{\prime}=-\mu-2 j-1, \\
& \left|j_{+}, k ; 1\right\rangle_{s \ell(2)}=\left(J_{0}^{+}\right)^{\mu+1}|j, \Lambda, k\rangle, \quad\left|j_{+}^{\prime}, k ; 1\right\rangle_{s \ell(2)}=\left(J_{0}^{+}\right)^{\mu^{\prime}+1}|j, \Lambda, k\rangle, \quad \mu^{\prime}=-\mu-2 j-1,
\end{aligned}
$$

which, in general, do not belong to $\mathcal{R}_{j, \Lambda, k}$, represent Verma highest-weight vectors with the respective parameters

$$
\begin{array}{ll}
j_{-}=j+\mu, & j_{-}^{\prime}=-j-\mu-1, \\
j_{+}=j+\mu+1+\frac{k}{2}, & j_{+}^{\prime}=-j-\mu+\frac{k}{2} .
\end{array}
$$


We now require that a singular vector exist in one of the 'continued' modules with the highestweight states from (11.40). Formulas (II.41) immediately give the following Lemma:

Lemma II.9 The following conditions are equivalent:

$$
\begin{aligned}
& j_{-}=\mathrm{j}^{ \pm}(r, s, k) \Longleftrightarrow j_{+}^{\prime}=\mathrm{j}^{\mp}(r, s, k) \Longleftrightarrow j_{-}^{\prime}=\mathrm{j}^{\mp}(r, s \mp 1, k), \\
& j_{-}^{\prime}=\mathrm{j}^{\mp}(r, s, k) \Longleftrightarrow j_{+}=\mathrm{j}^{ \pm}(r, s, k) \Longleftrightarrow j_{+}^{\prime}=\mathrm{j}^{\mp}(r, s \pm 1, k) .
\end{aligned}
$$

The existence of singular vectors in $\mathcal{R}_{j, \Lambda, k}$ is determined by whether and how many of states (III.40) admit a singular vector and/or belong to $\mathcal{R}_{j, \Lambda, k}$. Whenever one of these states belongs to the relaxed Verma module $\mathcal{R}_{j, \Lambda, k}$ - or, equivalently, $\mu$ or $\mu^{\prime}$ is an integer of the appropriate sign — we arrive at precisely the charged singular vector considered above (observe that (II.29) is nothing but ([I.39) with $\mu=p$ ). As regards singular vectors that might exist on states ([1.40), these must be the MFF vectors, which reduces the problem of explicitly constructing singular vectors in $\mathcal{R}_{j, \Lambda, k}$ to the MFF singular vectors.

We see from (II.41) and (II.39) that the condition for $\mathcal{R}_{j, \Lambda, k}$ to contain a relaxed Verma submodule reads as $\Lambda=\Lambda(r, s, j, k)$, where

$$
\Lambda(r, s, j, k)=-\left(j-\mathrm{j}^{-}(r, s, k)\right)\left(j-\mathrm{j}^{+}(r, s+1, k)\right) .
$$

Then, the state $\left(J_{0}^{-}\right)^{-\mu}|j, \Lambda(r, s, j, k), k\rangle_{s \ell(2)}$, which satisfies the Verma highest-weight conditions, has the spin $\mathrm{j}^{-}(r, s, k)$. Therefore the singular vector constructed on this state reads

$$
\mathcal{M F F}^{-}(r, s, k)\left(J_{0}^{-}\right)^{-\mathrm{j}^{-}(r, s, k)+j}|j, \Lambda(r, s, j, k), k\rangle_{s \ell(2)},
$$

where $\mathcal{M F F}^{-}$is the singular vector operator (read off by dropping the highest-weight state in (III.14)). This Verma-module singular vector has to be mapped back to the original relaxed Verma module $\mathcal{R}_{j, \Lambda(r, s, j, k), k}$, where it will become a relaxed singular vector. In particular, no non-integral powers of $J_{0}^{-}$should remain, which is achieved by acting on (II.44) with $\left(J_{0}^{-}\right)^{\mathrm{j}^{-}(r, s, k)-j+N}$, where $N$ is an integer, and making use of (II.12). However, to be left with only positive integral powers after the rearrangements, the integer $N$ has to be $\geq r+r s$. We thus define

$$
\left|\Sigma^{-}(r, s, j, k)\right\rangle=\left(J_{0}^{-}\right)^{\mathrm{j}^{-}(r, s, k)-j+r+r s} \mathcal{M F F}^{-}(r, s, k)\left(J_{0}^{-}\right)^{-\mathrm{j}^{-}(r, s, k)+j}|j, \Lambda(r, s, j, k), k\rangle_{s \ell(2)}
$$

as a 'canonical' representative of the relaxed singular vector in $\mathcal{R}_{j, \Lambda(r, s, j, k), k}$. 
Similarly, the state $\left(J_{0}^{+}\right)^{\mu+1}|j, \Lambda(r, s, j, k), k\rangle$ with $\mu$ as in (【I.41), i.e., $\left(J_{0}^{+}\right)^{1+j^{+}(r, s+1, k)-j}$. $|j, \Lambda(r, s, j, k), k\rangle_{s \ell(2)}$, is a Verma highest-weight state twisted by the spectral flow transform with $\theta=1$. We then construct the corresponding MFF singular vector and finally map it back to the relaxed Verma module. In this way, the singular vector in the relaxed Verma module becomes

$$
\begin{aligned}
& \left|\Sigma^{+}(r, s, j, k)\right\rangle= \\
& \quad=\left(J_{0}^{+}\right)^{-1-\mathrm{j}^{+}(r, s+1, k)+j+r+r s} \mathcal{M F F}^{+, 1}(r, s, k)\left(J_{0}^{+}\right)^{1+\mathrm{j}^{+}(r, s+1, k)-j}|j, \Lambda(r, s, j, k), k\rangle_{s \ell(2)},
\end{aligned}
$$

where $\mathcal{M F F}^{+, \theta}$ is the spectral flow transform (匹I.7) of the singular vector operator $\mathcal{M F F}^{+}$.

The construction can be illustrated in the following extremal diagram:

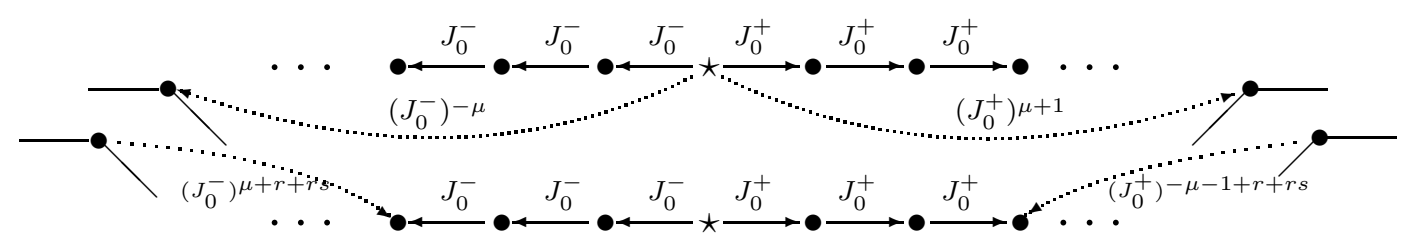

Every point in the lower floor is annihilated by the operators $J_{\geq 1}^{ \pm, 0}$ and, thus, satisfies the relaxed highest-weight conditions. The Verma module shown on the left contains a submodule. The Verma module and its submodule on the right are 'rotated' by the spectral flow transform with $\theta=1$. The Verma modules are to be thought of as disconnected from the extremal diagram of the relaxed Verma module $\mathcal{R}_{j, \Lambda(r, s, j, k), k}$, since they do not, in general, belong to $\mathcal{R}_{j, \Lambda(r, s, j, k), k}$ (the mappings shown in dotted lines are given by complex powers of the generators).

Expressions ([1.46), ([1.45), and (ㅍ.30) give explicit constructions for singular vectors that can appear in relaxed Verma modules. In order to describe all degenerate cases with several singular vectors in $\mathcal{R}_{j, \Lambda, k}$ appearing simultaneously, we first classify the different degeneration patterns. Recall that, while the module $\mathcal{R}_{j, \Lambda, k}$ is irreducible for general $j, \Lambda$, and $k$, singular vectors appear in codimension 1, when these parameters are related by 1 equation. In the general case in codimension $1, j$ and $k$ are still arbitrary. To describe degeneration patterns in higher codimensions, we divide the space of the highest-weights $O \equiv\{(j, \Lambda, k)\}$ into a union $\cup_{i} O_{i}=O$ of such $O_{i}$ that $O_{i} \cap O_{j}=\emptyset$ and all modules $\mathcal{R}_{j, \Lambda, k}$ with $(j, \Lambda, k) \in O_{i}$ have the same type of degeneration for every $i$.

For brevity, we will say that a highest-weight state admits a singular vector whenever the Verma module generated from that state contains this singular vector and that a highest-weight 
state admits no singular vectors if no singular vectors exist in the Verma module. The sets $O_{1}$, $\mathrm{O}_{2}, \ldots, \mathrm{O}_{n}$ with the above properties can be singled out by requiring that a certain number of the states from ([I.40) belong to the relaxed Verma module or/and admit singular vectors. The list of all possible cases is as follows:

- codimension 0:

$O_{\emptyset}$ None of states ([I.40) belong to the module $\mathcal{R}_{j, \Lambda, k}$ and at least one of states ([I.40) admits no singular vectors.

- codimension 1:

$O_{c}$ Exactly one of states ([11.40) belongs to the module $\mathcal{R}_{j, \Lambda, k}$ and none of states ([1I.40) admit a singular vector.

$O_{r}$ One of states ([I.40) admits precisely one singular vector, each of the other states ([I.40) admits at least one singular vector and none of states (II.40) belong to the module $\mathcal{R}_{j, \Lambda, k}$.

- codimension 2 :

$O_{r r}$ Each of states (II.40) admits at least two singular vectors and none of the states from ([1.40) belong to $\mathcal{R}_{j, \Lambda, k}$.

$O_{c c}$ Precisely one of the states from each line in (II.40) belongs to $\mathcal{R}_{j, \Lambda, k}$ and none of these two states admit a singular vector;

$O_{r c}$ One of the states from ([I.40) belongs to $\mathcal{R}_{j, \Lambda, k}$ and admits precisely one singular vector; none of states ([1.40) admit two different singular vectors; no two states from different lines in ([I.40) belong to $\mathcal{R}_{j, \Lambda, k}$.

- codimension 3:

$O_{r r c}$ One of the states from (II.40) belongs to $\mathcal{R}_{j, \Lambda, k}$ and admits at least two singular vectors; no two states from different lines in (II.40) belong to $\mathcal{R}_{j, \Lambda, k}$.

$O_{r c c}$ Precisely one of the states from each line in ([11.40) belongs to $\mathcal{R}_{j, \Lambda, k}$; each of these two states admits at least one singular vector. 
The sets $O_{i}$ from this list can be singled out by systems of equations. Thus, the $O_{i}$ of codimension 1 can be thought of as hyperplanes with certain lines eliminated, while the $O_{i}$ of codimension 2 are lines with some (rational) points eliminated, and the $O_{i}$ sets of codimension 3 are discrete collections of points.

We will describe degenerate cases in the order given in the above list. To begin with, observe that the completeness of the above list implies that the relaxed Verma module $\mathcal{R}_{j, \Lambda, k}$ is irreducible if and only if $(j, \Lambda, k) \in O_{\emptyset}$. Further, the cases where the relaxed Verma module $\mathcal{R}_{j, \Lambda, k}$ contains precisely one submodule are described in the following theorem:

\section{Theorem II.10}

I. The highest-weight $(j, \Lambda, k)$ of the relaxed Verma module $\mathcal{R}_{j, \Lambda, k}$ belongs to the set $O_{c}$ if and only if $\Lambda=\Lambda_{\mathrm{ch}}(n, j)$ and

$(n, j, k) \in(\mathbb{Z} \times \mathbb{C} \times \mathbb{C}) \backslash\left\{\left(n, \frac{1}{2}(r-2 n-1)-\frac{1}{2}(k+2) s, k\right) \mid r, s \in \mathbb{Z}, r \neq 0, r \cdot s \geq 0, n \in \mathbb{Z}, k \in \mathbb{C}\right\}$.

The highest-weight of the relaxed Verma module $\mathcal{R}_{j, \Lambda, k}$ belongs to the set $O_{r}$ if and only if $\Lambda=\Lambda(r, s, j, k)$ with

$$
(r, s, j, k) \in(\mathbb{N} \times \mathbb{N} \times \mathbb{C} \times(\mathbb{C} \backslash \mathbb{Q}) \bigcup \mathbb{Y}) \backslash \mathbb{X}(\mathbb{C})
$$

where

$$
\begin{gathered}
\mathbb{Y}=\left\{\left(r, s, j,-\frac{p}{q}-2\right) \mid 1 \leq r \leq p, 1 \leq s \leq q, p, q \in \mathbb{N}, j \in \mathbb{C}\right\} \bigcup \\
\left\{\left(r, 1, j,-\frac{p}{q}-2\right) \mid p+1 \leq r \leq 2 p, p, q \in \mathbb{N}, j \in \mathbb{C}\right\},
\end{gathered}
$$

and, for any set $\mathcal{P} \subset \mathbb{C}$, we have defined

$$
\mathbb{X}(\mathcal{P})=\left\{\left(r, s, \frac{1}{2}( \pm r-2 n-1) \mp \frac{1}{2}(k+2) s, k\right) \mid n \in \mathbb{Z}, r, s \in \mathbb{N}, k \in \mathcal{P}\right\} .
$$

II. In the $O_{c}$ case, the module $\mathcal{R}_{j, \Lambda_{\mathrm{ch}}(n, j), k}$ contains precisely one submodule (which is isomorphic to a Verma module) generated from singular vector ([1.30).

III. In the $O_{r}$ case, the module $\mathcal{R}_{j, \Lambda(r, s, j, k), k}$ contains precisely one submodule (which is isomorphic to a relaxed Verma module). Expressions ([1.45) and (II.46) evaluate as elements

\footnotetext{
${ }^{3}$ Whenever we write $k+2=\frac{p}{q}$ with $p \in \mathbb{Z}, q \in \mathbb{N}$, we always assume $p$ and $q$ to be coprime.
} 
of the relaxed Verma module $\mathcal{R}_{j, \Lambda}(r, s, j, k), k$ and satisfy the relaxed-highest-weight conditions

$$
\begin{aligned}
& J_{\geq 1}^{+}\left|\Sigma^{ \pm}(r, s, j, k)\right\rangle=J_{\geq 1}^{0}\left|\Sigma^{ \pm}(r, s, j, k)\right\rangle=J_{\geq 1}^{-}\left|\Sigma^{ \pm}(r, s, j, k)\right\rangle=0, \\
& J_{0}^{-} J_{0}^{+}\left|\Sigma^{ \pm}(r, s, j, k)\right\rangle=\Lambda^{ \pm}(r, s, j, k)\left|\Sigma^{ \pm}(r, s, j, k)\right\rangle, \\
& J_{0}^{0}\left|\Sigma^{ \pm}(r, s, j, k)\right\rangle=(j \pm r s)\left|\Sigma^{ \pm}(r, s, j, k)\right\rangle,
\end{aligned}
$$

where

$$
\Lambda^{ \pm}(r, s, j, k)=\frac{1}{4}(r+(k+2) s-2 r s \mp(1+2 j))(r+(k+2) s+2 r s \pm(1+2 j)) .
$$

Each of the singular vectors $\Sigma^{ \pm}(r, s, j, k)$ generates the entire relaxed Verma submodule; in particular, $J_{0}^{ \pm}$-descendants of ([1.45) and (11.46) are on the same extremal subdiagram and coincide up to numerical factors whenever they are in the same grade:

$$
\begin{aligned}
c_{+}(i, j, k)\left(J_{0}^{+}\right)^{i}\left|\Sigma^{-}(r, s, j, k)\right\rangle & =c_{-}(i, j, k)\left(J_{0}^{-}\right)^{2 r s-i}\left|\Sigma^{+}(r, s, j, k)\right\rangle, & & 0 \leq i \leq 2 r s, \\
c_{+}(i, j, k)\left(J_{0}^{+}\right)^{i}\left|\Sigma^{-}(r, s, j, k)\right\rangle & =c_{-}(i, j, k)\left(J_{0}^{+}\right)^{i-2 r s}\left|\Sigma^{+}(r, s, j, k)\right\rangle, & & i>2 r s, \\
c_{+}(i, j, k)\left(J_{0}^{-}\right)^{-i}\left|\Sigma^{-}(r, s, j, k)\right\rangle & =c_{-}(i, j, k)\left(J_{0}^{-}\right)^{-i+2 r s}\left|\Sigma^{+}(r, s, j, k)\right\rangle, & & i<0,
\end{aligned}
$$

where the numerical coefficients $c_{ \pm}(i, j, k)$ are ( $r$-and $s$-dependent) polynomials in $j$ and $k$.

Thus, (II.45) and (II.46) are, in general, different representatives of the same singular vector, since they generate the same submodule. This is not necessarily so in higher codimensions. To see what can happen there, consider again diagram (II.47). There, the Verma modules that contain singular vectors are shown as disconnected pieces, since they are related to the Verma module by mappings with complex powers of $J_{0}^{ \pm}$. However, when one or both of the roots of (II.39) becomes an integer, one or both of these Verma modules are no longer disconnected from the extremal diagram of the relaxed Verma module. Then the Verma highest-weight conditions are encountered in the extremal diagram and restrict the possibilities to travel over the states in the lower floor in ([1.47), since some of the states in ([1.53) might vanish. For example, it may be impossible to obtain the $\Sigma^{+}$representative of the singular vector as a $J_{0}^{+}$-descendant of $\Sigma^{-}$, or/and to obtain $\Sigma^{-}$as a $J_{0}^{-}$-descendant of $\Sigma^{+}$. These cases in codimension $\geq 2$ describe simultaneous occurrence of several singular vectors, and will be considered in what follows. We now proceed to codimension 2 .

Codimension-2 cases. In codimension 2, we have three cases of a simultaneous occurrence of two singular vectors, which are described in the three following Theorems. The following 
Lemma governs the appearance of Verma submodules in a relaxed Verma module. If one puts $\Lambda^{ \pm}(r, s, j, k)$ in ([1.52) equal to $\Lambda_{\mathrm{ch}}(N, j \pm r s)$ (see Eq. (II.29)), one discovers that the condition $\Lambda^{ \pm}(r, s, j, k)=\Lambda_{\mathrm{ch}}(N, j \pm r s)$ implies a similar relation for $\Lambda(r, s, j, k)$, and therefore charged singular vectors in the lower floor occur synchronously with charged singular vectors in the top floor of the extremal diagram. In this way, we arrive at

Lemma II.11 Let $\mathcal{R}$ be the relaxed Verma module $\mathcal{R} \equiv \mathcal{R}_{j, \Lambda, k}$.

1. Let $\mathcal{R} \supset \mathcal{R}^{\prime}$ and $\mathcal{R} \supset \mathcal{C}$, where $\mathcal{R}^{\prime}$ is a relaxed Verma submodule and $\mathcal{C}$ is a (twisted) Verma module generated from a charged singular vector in $\mathcal{R}$, and let $\mathcal{C}$ be maximal in the sense that $\mathcal{R} \supset \mathcal{C}^{\prime \prime} \supset \mathcal{C}$, where $\mathcal{C}^{\prime \prime}$ is any other (twisted) Verma submodule, implies $\mathcal{C}^{\prime \prime}=\mathcal{C}$, Then $\mathcal{R}^{\prime} \cap \mathcal{C}=\mathcal{C}^{\prime} \neq\{0\}$, where $\mathcal{C}^{\prime}$ is a (twisted) Verma module generated from a charged singular vector in $\mathcal{R}^{\prime}$.

2. Conversely, if $\mathcal{R} \supset \mathcal{R}^{\prime}$, where $\mathcal{R}^{\prime}$ is a relaxed Verma module and $\mathcal{R}^{\prime} \supset \mathcal{C}^{\prime}$, where $\mathcal{C}^{\prime}$ is a submodule generated from a charged singular vector in $\mathcal{R}^{\prime}$, then $\mathcal{R} \supset \mathcal{C} \supset \mathcal{C}^{\prime}$, where $\mathcal{C}$ is a submodule generated from a charged singular vector in $\mathcal{R}$, and $\mathcal{R} \supset \mathcal{C}^{\prime \prime} \supset \mathcal{C} \Longrightarrow \mathcal{C}^{\prime \prime}=\mathcal{C}$.

3. If $\mathcal{R} \supset \mathcal{C}^{\prime}$, where $\mathcal{C}^{\prime}$ is a (twisted) Verma module, there exists a (twisted) Verma submodule $\mathcal{C} \subset \mathcal{R}$ such that the embedding is given by a charged singular vector and $\mathcal{R} \supset \mathcal{C}^{\prime \prime} \supset \mathcal{C} \Longrightarrow$ $\mathcal{C}^{\prime \prime}=\mathcal{C}$.

This Lemma is a necessary tool in the proof of the following three Theorems. Consider first the occurrence of two different relaxed singular vectors.

We call a submodule primitive if it is not a sum of two other submodules.

Theorem II.12 The highest-weight of the module $\mathcal{R}_{j, \Lambda, k}$ belongs to the set $O_{r r}$ if and only if $\Lambda=\Lambda(r, s, j, k)$ with

$$
(r, s, j, k) \in \mathbb{N} \times \mathbb{N} \times \mathbb{C} \times \mathbb{Q} \backslash(\mathbb{X}(\mathbb{Q}) \cup \mathbb{Y})
$$

where $\mathbb{Y}$ and $\mathbb{X}(\mathbb{Q})$ are as in $(\mathbb{I I . 4 9})$ and ([I.50). Then,

1. any primitive submodule in $\mathcal{R}_{j, \Lambda\left(r, s, j, \frac{p}{q}\right), \frac{p}{q}}$ is a relaxed Verma module. It is generated from $\left|\Sigma^{-}\left(r, s, j, \frac{p}{q}\right)\right\rangle$ or, equivalently, from $\left|\Sigma^{+}\left(r, s, j, \frac{p}{q}\right)\right\rangle$ with the same $r$ and $s$; 
2. for any primitive submodule $\mathcal{R}^{\prime} \subset \mathcal{R}_{j, \Lambda\left(r, s, j, \frac{p}{q}\right), \frac{p}{q}}$, there exists a singular vector $e \equiv \mathcal{E}$. $\left|\mathrm{j}^{-}\left(r, s, \frac{p}{q}\right), \frac{p}{q}\right\rangle_{s \ell(2)}$ in the Verma module $\mathcal{M}_{\mathbf{j}^{-}\left(r, s, \frac{p}{q}\right), \frac{p}{q}}$ such that $\mathcal{R}^{\prime}$ is generated from a relaxed singular vector

$$
\left(J_{0}^{-}\right)^{\mathrm{j}^{-}(r, s, k)-j+r+r s} \mathcal{E}\left(J_{0}^{-}\right)^{-\mathrm{j}^{-}(r, s, k)+j}|j, \Lambda(r, s, j, k), k\rangle_{s \ell(2)}
$$

3. conversely, for any singular vector $e$ in $\mathcal{M}_{\mathbf{j}^{-}\left(r, s, \frac{p}{q}\right), \frac{p}{q}}$, of the form $e=\left|\operatorname{MFF}\left(r, s, \frac{p}{q}\right)\right\rangle^{+}$, $r \geq 1, s \geq 2$, or $e=\left|\operatorname{MFF}\left(r, s, \frac{p}{q}\right)\right\rangle^{-}, r, s \geq 1$, there exists a relaxed Verma submodule in $\mathcal{R}_{j, \Lambda\left(r, s, j, \frac{p}{q}\right), \frac{p}{q}}$ generated by the relaxed singular vector (II.54);

4. two different singular vectors $e_{1}$ and $e_{2}$ in $\mathcal{M}_{\mathbf{j}^{-}\left(r, s, \frac{p}{q}\right), \frac{p}{q}}$ correspond in this way to the same relaxed Verma submodule in $\mathcal{R}$ if and only if one of the $e_{i}$ is the $\operatorname{MFF}^{+}\left(a \geq 1,1, \frac{p}{q}\right)$ singular vector in the module generated from the other.

In this case, therefore, the structure of the relaxed Verma module $\mathcal{R}_{j, \Lambda\left(r, s, j, \frac{p}{q}\right), \frac{p}{q}}$ is determined by the structure of the Verma module $\mathcal{M}_{\mathbf{j}-\left(r, s, \frac{p}{q}\right), \frac{p}{q}}$.

Now, consider the case where both Verma modules shown in (ㅍ.47) actually belong to the relaxed extremal diagram. It is described in the following Theorem.

Theorem II.13 The highest-weight of the relaxed Verma module $\mathcal{R}_{j, \Lambda, k}$ belongs to the set $O_{c c}$ of the list of degeneration patterns if and only if $j=j_{\mathrm{cc}}(n, m), \Lambda=\Lambda_{\mathrm{cc}}(n, m)$, where

$$
j_{\mathrm{cc}}(n, m)=-\frac{1}{2}(1+m+n), \quad \Lambda_{\mathrm{cc}}(n, m)=-m n,
$$

and

$(n, m, k) \in\left((-\mathbb{N}) \times \mathbb{N}_{0} \times(\mathbb{C} \backslash \mathbb{Q})\right) \bigcup\left\{\left(n, m,-\frac{p}{q}-2 \mid n \in-\mathbb{N}, m \in \mathbb{N}_{0}, p, q \in \mathbb{N}, 1 \leq m-n \leq q\right\}\right.$

Then the relaxed Verma module $\mathcal{R}_{j, \Lambda, k}$ contains the Verma submodule $\mathcal{C}_{-} \approx \mathcal{M}_{\frac{1}{2}(n-m-1), k}$ generated from the charged singular vector $|C(n, j, k)\rangle$ and the twisted Verma submodule $\mathcal{C}_{+} \approx$ $\mathfrak{M}_{\frac{1}{2}(1+m-n+k), k ; 1}$ generated from the charged singular vector $|C(m, j, k)\rangle$. The maximal submodule is $\mathcal{C}_{+} \cup \mathcal{C}_{-}$, and $\mathcal{C}_{+} \cap \mathcal{C}_{-}=\emptyset$.

The third possibility in codimension 2, i.e. the case $O_{r c}$ from the list, is when one of the states ([I.40) belongs to the module $\mathcal{R}_{j, \Lambda, k}$ (hence this module contains a charged singular vector) and at the same time one of states (II.40) admits a singular vector. This case is more involved and is analyzed in what follows. 
According to Lemma II.11, the Verma submodule growing out of the charged singular vector on the top floor of the extremal diagram necessarily has a submodule generated from a singular vector $\operatorname{MFF}^{ \pm}(r, s, k)$, and this submodule coincides with the submodule built on the charged singular vector in the lower floor of the extremal diagram, e.g.

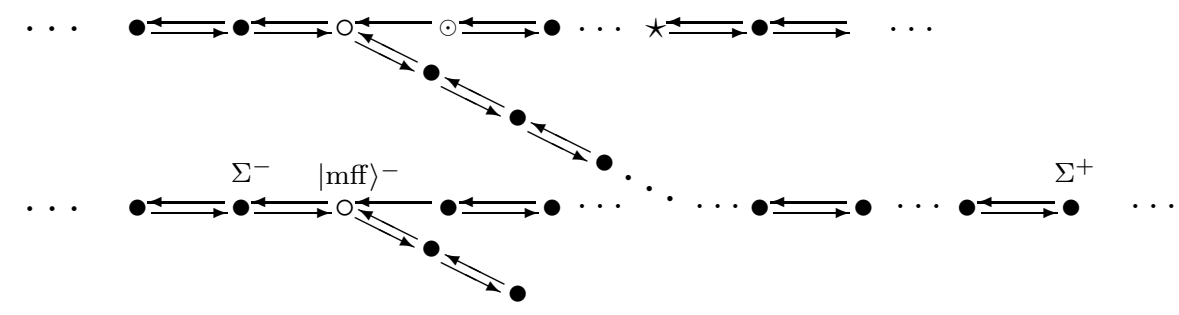

As we saw in ([11.51), the $\Sigma^{-}$representative of the relaxed singular vector is at the distance of rs arrows on the left from the 'center' (the $\star$ ), while $\Sigma^{+}$is at the same distance on the right. It may happen that $\Sigma^{-}$would be inside the Verma submodule. In that case, the entire extremal diagram of the submodule cannot be generated by $J_{0}^{ \pm}$-descendants of $\Sigma^{-}$. Unless we are in codimension 3, however, the entire diagram of the submodule would still be generated from $\Sigma^{+}$. In particular, following the arrows from $\Sigma^{+}$to $\Sigma^{-}$into the Verma submodule makes the two representatives proportional. This is described more precisely in the following Theorem.

Theorem II.14 The highest-weight of the relaxed Verma module $\mathcal{R}_{j, \Lambda, k}$ belongs to the set $O_{r c}$ of the list of degeneration patterns if and only if $j=j_{\mathrm{rc}}^{\sigma}(r, s, n, k)$ and $\Lambda=\Lambda_{\mathrm{rc}}^{\sigma}(r, s, n, k)$, where $\sigma \in\{+,-\}$,

$$
\begin{aligned}
& j_{\mathrm{rc}}^{ \pm}(r, s, n, k)=-n-\frac{1}{2} \pm \frac{1}{2}(r-(k+2) s), \\
& \Lambda_{\mathrm{rc}}^{ \pm}(r, s, n, k)=-n^{2} \pm n(r-(k+2) s)
\end{aligned}
$$

and

$$
(\sigma, r, s, n, k) \in(\{ \pm\} \times \mathbb{N} \times \mathbb{N} \times \mathbb{Z} \times(\mathbb{C} \backslash \mathbb{Q})) \bigcup \mathbb{A}(\mathbb{C} \backslash \mathbb{Q}) \bigcup \mathbb{B}
$$

where

$$
\begin{aligned}
\mathbb{A}(\mathcal{P})= & (\{+\}, r, 0, n, k) \mid r \in \mathbb{N}, n \in-\mathbb{N}, k \in \mathcal{P}\} \bigcup\left\{(\{-\}, r, 0, n, k) \mid r \in \mathbb{N}, n \in \mathbb{N}_{0}, k \in \mathcal{P}\right\}, \\
\mathbb{B}=\left\{\left(\{+\}, r, s, n,-\frac{p}{q}-2\right) \mid n \in-\mathbb{N}, p, q \in \mathbb{N}, 1 \leq r \leq p, 0 \leq s \leq q-1\right\} \bigcup & (\text { II.60) } \\
& \left\{\left(\{-\}, r, s, n,-\frac{p}{q}-2\right) \mid n \in \mathbb{N}_{0}, p, q \in \mathbb{N}, 1 \leq r \leq p, 0 \leq s \leq q-1\right\} .
\end{aligned}
$$

Then, the relaxed Verma module $\mathcal{R}_{j, \Lambda, k}$ contains a relaxed Verma submodule $\mathcal{R}^{\prime}$ generated from the singular vector $\left|\Sigma^{+}\left(r, s, j_{\mathrm{rc}}^{ \pm}(r, s, n, k), k\right)\right\rangle$ (if $n<0$ ) or $\left|\Sigma^{-}\left(r, s, j_{\mathrm{rc}}^{ \pm}(r, s, n, k), k\right)\right\rangle$ (if $n \geq 0)$; the module $\mathcal{R}_{j, \Lambda, k}$ contains also a Verma submodule $\mathcal{C} \approx \mathcal{M}_{\mathbf{j}^{ \pm}(r, s, k), k}($ if $n<0)$ or 
a twisted Verma submodule $\mathcal{C} \approx \mathfrak{M}_{\mathfrak{j}^{ \pm}(r, s, k), k ; 1}$ (if $n \geq 0$ ) generated from the charged singular vector $|C(n, j, k)\rangle$ in either case. The maximal submodule in $\mathcal{R}_{j, \Lambda, k}$ is $\mathcal{C} \cup \mathcal{R}^{\prime}$. The intersection $\mathcal{C}^{\prime} \equiv \mathcal{C} \cap \mathcal{R}^{\prime}$ is a Verma module (if $n<0$ ) or a twisted Verma module (if $n \geq 0$ ) generated from a charged singular vector $|c\rangle \in \mathcal{R}^{\prime}$, which is also a singular vector in $\mathcal{C}$ :

$$
|c\rangle=\left\{\begin{aligned}
|\mathrm{mff}\rangle^{ \pm} & =\mathcal{M F F}^{ \pm}\left(r, s+\frac{1}{2} \pm \frac{1}{2}, k\right)\left|C\left(n, j_{\mathrm{rc}}^{ \pm}(r, s, n, k), k\right)\right\rangle, & & n<0 ; \\
|\mathrm{mff}\rangle^{\mp, 1} & =\mathcal{M} \mathcal{F} \mathcal{F}^{\mp, 1}\left(r, s+\frac{1}{2} \pm \frac{1}{2}, k\right)\left|C\left(n, j_{\mathrm{rc}}^{ \pm}(r, s, n, k), k\right)\right\rangle, & & n \geq 0 .
\end{aligned}\right.
$$

Remark II.15 When the intersection $\mathcal{C}^{\prime}=\mathcal{C} \cap \mathcal{R}^{\prime}$ is generated by the singular vector $\mathcal{M F F}^{-}(r, s, k)\left|C\left(n, j_{\mathrm{rc}}^{-}(r, s, n, k), k\right)\right\rangle$ with $n<0$ and $|n| \leq r(s+1)$, the relaxed singular vector $\left|\Sigma^{-}\left(r, s, j_{\mathrm{rc}}^{-}(r, s, n, k), k\right)\right\rangle$ belongs to the Verma submodule $\mathcal{C}^{\prime}$ and thus cannot generate the entire relaxed submodule $\mathcal{R}$ :

$$
\left|\Sigma^{-}\left(r, s, j_{\mathrm{rc}}^{-}(r, s, n, k), k\right)\right\rangle=\left(J_{0}^{-}\right)^{r+r s+n}|c\rangle .
$$

The relative positions of $\Sigma^{-}$and $\Sigma^{+}$in the extremal diagram are as follows:t

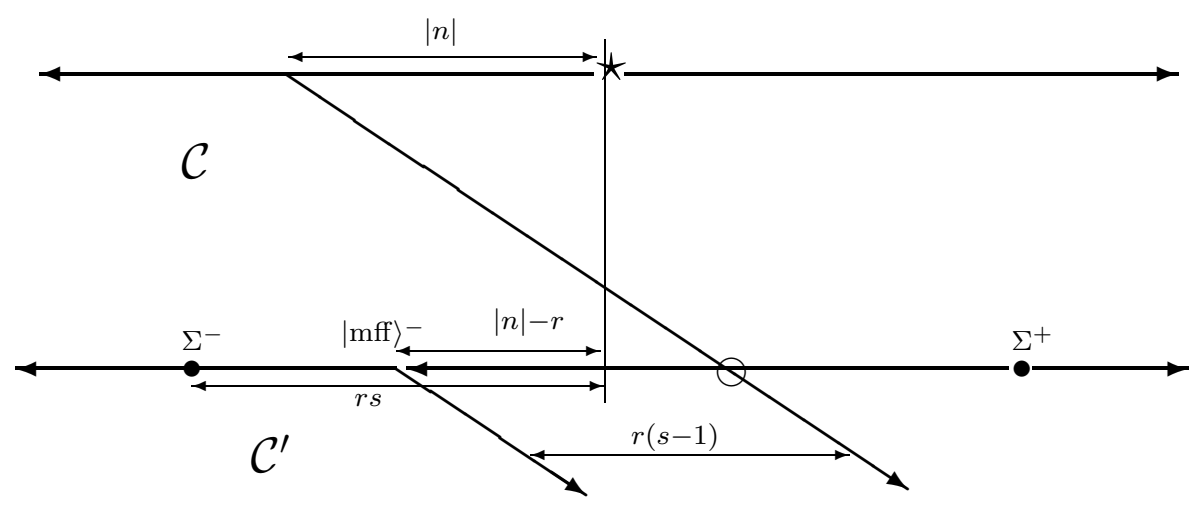

where length $|n|-r$ shown as if positive. Under the conditions of the Theorem, $\Sigma^{+}$still has infinitely many descendants with respect to both $J_{0}^{-}$and $J_{0}^{+}$.

Going 'past the highest-weight'. The above concludes the analysis of codimension-2 cases. In the subsequent analysis of the codimension 3 , however, it will be very useful to know more subtle properties of the chosen representatives of singular vectors. As we saw in (II.63), descendants of $\Sigma^{-}$may not cover a part of the extremal subdiagram because of the Verma highestweight conditions satisfied at $|\mathrm{mff}\rangle^{-}$. In applications, however, one needs to know whether or

\footnotetext{
${ }^{4}$ At the point marked with a circle, two lines do not 'intersect', i.e., they lead to different vectors in the same grade. The same applies to the diagrams below, which will not be stated explicitly however.
} 
not a given Verma submodule in a relaxed Verma module is actually generated from a charged singular vector in some relaxed Verma submodule.]

Thus, given a Verma highest-weight state $|X\rangle$, which satisfies $J_{0}^{+}|X\rangle=0$, we are interested in whether there exists a state $|Y\rangle$ in the relaxed Verma module such that $|X\rangle=J_{0}^{-}|Y\rangle$, or, to put it differently, whether $\left(J_{0}^{-}\right)^{-1}|X\rangle$ can be defined as an element of the relaxed Verma module.

We now answer this question for vectors (II.45) and (II.46). First of all, whenever $\Sigma^{-}$is in the Verma submodule $\mathcal{C}^{\prime}$ generated by $\operatorname{MFF}^{-}(r, s, k)$, we consider instead of $\Sigma^{-}$the highestweight vector of that submodule:

$$
\left.\left|\widehat{\Sigma}^{-}\left(r, s, j_{\mathrm{rc}}^{-}(r, s, n, k), k\right)\right\rangle \equiv|\mathrm{mff}\rangle^{-}=\mathcal{M F F}^{-}(r, s, k)\left|j_{\mathrm{rc}}^{-}(r, s, n, k), \Lambda_{\mathrm{rc}}^{-}(r, s, n, k), k\right| n\right\rangle_{s \ell(2)}
$$

of which $\Sigma^{-}$is a $J_{0}^{-}$-descendant.

The vector $\left|\widehat{\Sigma}^{-}\left(r, s, j_{\mathrm{rc}}^{-}(r, s, n, k), k\right)\right\rangle$ is the farthest right one can reach by acting with $J_{0}^{+}$ on the original singular vector $\left|\Sigma^{-}\left(r, s, j_{\mathrm{rc}}^{-}(r, s, n, k), k\right)\right\rangle$. To map it farther right, to the outside of the Verma submodule $\mathcal{C}^{\prime}$, one employs the operator $\left(J_{0}^{-}\right)^{-1}$, which is understood as one of the 'continued' operators from ([I.11)-(II.12):

$$
\left|\widetilde{\Sigma}^{-}\left(r, s, j_{\mathrm{rc}}^{-}(r, s, n, k), k\right)\right\rangle=\left(J_{0}^{-}\right)^{-1}\left|\widehat{\Sigma}^{-}\left(r, s, j_{\mathrm{rc}}^{-}(r, s, n, k), k\right)\right\rangle .
$$

This can be pictured as

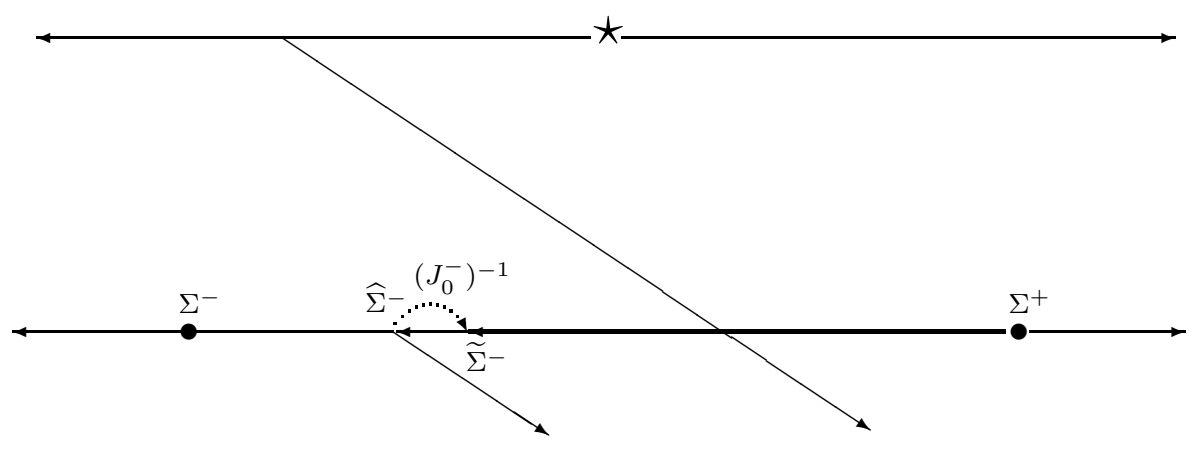

The conditions for $\left|\widetilde{\Sigma}^{-}\left(r, s, j_{\mathrm{rc}}^{-}(r, s, n, k), k\right)\right\rangle$ to exist are given by the following Lemma. Let

$$
f(r, s, n, k)= \begin{cases}\prod_{m=0}^{2 r-n}(r-m-n-(k+2) s), & 1 \leq n \leq 2 r, \\ 1, & n \geq 2 r+1 .\end{cases}
$$

\footnotetext{
${ }^{5}$ Both cases can occur, and the distinction between the two possibilities is crucial for the correct classification of degenerate cases, and also, for the construction of embedding diagrams, etc.
} 
Lemma II.16 In the relaxed Verma module $\mathcal{R}_{j_{\mathrm{rc}}^{-}(r, s, n, k), \Lambda_{\mathrm{rc}}^{-}(r, s, n, k), k}$ with $n<0$ and $|n| \leq r(s+1)$,

I. the vector

$$
\left.\left|\widetilde{\Sigma}^{-}\left(r, s, j_{\mathrm{rc}}^{-}(r, s, n, k), k\right)\right\rangle=\left(J_{0}^{-}\right)^{-1} \mathcal{M F}^{\mathcal{F F}^{-}}(r, s, k)\left|j_{\mathrm{rc}}^{-}(r, s, n, k), \Lambda_{\mathrm{rc}}^{-}(r, s, n, k), k\right| n\right\rangle_{s \ell(2)}
$$

exists if and only if $f(r, s,|n|, k) \neq 0$, in which case it is a relaxed singular vector;

II. Under the conditions of Theorem II.14, a $J_{0}^{+}$-descendant of $\left|\Sigma^{+}\left(r, s, j_{\mathrm{rc}}^{-}(r, s, n, k), k\right)\right\rangle$ is proportional to vector (ㅍ.68):

$$
\left(J_{0}^{-}\right)^{r s-n-r-1}\left|\Sigma^{+}\left(r, s, j_{\mathrm{rc}}^{-}(r, s, n, k), k\right)\right\rangle=(-1)^{r} a(r, s, n, k)\left|\widetilde{\Sigma}^{-}\left(r, s, j_{\mathrm{rc}}^{-}(r, s, n, k), k\right)\right\rangle,
$$

where

$$
\begin{aligned}
a(r, s, n, k) & =\frac{\prod_{i=0}^{r(s+1)-1}\left(\Lambda_{\mathrm{rc}}^{-}(r, s, n, k)-i(i+1)-2 i j_{\mathrm{rc}}^{-}(r, s, n, k)\right)}{\prod_{i=0}^{2 r-1}(n-i-r(s-1))} \\
& =\prod_{i=0}^{r(s+1)-1}(i-r-n+(k+2) s) \prod_{i=0}^{r(s-1)-1}(n-i) .
\end{aligned}
$$

To prove the Lemma, we observe that the action of $\left(J_{0}^{-}\right)^{-1}$ is given by ([I.12) and by Eqs. ([I.23) rewritten as

$$
\begin{array}{rlrl}
\left.\left(J_{0}^{-}\right)^{-1}|j, \Lambda, k| m\right\rangle_{s \ell(2)} & =|j, \Lambda, k| m+1\rangle_{s \ell(2)}, & & m \leq-1 \\
\left.\left(J_{0}^{-}\right)^{-1}|j, \Lambda, k| m\right\rangle_{s \ell(2)} & \left.=\frac{1}{\Lambda-m(m+1)-2 m j}|j, \Lambda, k| m+1\right\rangle_{s \ell(2)}, & m \geq 0 .
\end{array}
$$

This applies unless the denominator in the last formula vanishes. A simple analysis of the relative $J_{0}^{0}$-charge of $\left|\widehat{\Sigma}^{-}\left(r, s, j_{\mathrm{rc}}^{-}(r, s, n, k), k\right)\right\rangle$ shows that $f(r, s,-n, k)$ is precisely the function responsible for the existence of $\left|\widetilde{\Sigma}^{-}\left(r, s, j_{\mathrm{rc}}^{-}(r, s, n, k), k\right)\right\rangle$ : to evaluate ([I.65), one uses Eqs. (II.12) and then, as negative powers of $J_{0}^{-}$reach the highest-weight state, one applies (II.71) the appropriate number of times; the relevant factors from the denominators are precisely the above $f(r, s,-n, k)$, whence the lemma follows.

Thus, whenever state ([1.65) exists, it is proportional to a $J_{0}^{-}$-descendant of $\Sigma^{+}$.

Codimension 3. The codimension-3 cases are more involved than the previous ones. Let us begin with the case where a further degeneration occurs in Theorem $\mathbb{1 1 . 1 2}$. 
Equivalence between $\widehat{s \ell}(2)$ and $N=2$ representations

Theorem II.17 The highest-weight of the relaxed Verma module $\mathcal{R}_{j, \Lambda, k}$ belongs to the set $O_{\text {rrc }}$ of the list of degeneration patterns if and only if $j=j_{\mathrm{rc}}^{\sigma}(r, s, n, k)$ and $\Lambda_{\mathrm{rc}}^{+}(r, s, n, k)$ as in (II.58), where

$$
(\sigma, r, s, n, k) \in(\{ \pm\} \times \mathbb{N} \times \mathbb{N} \times \mathbb{Z} \times \mathbb{Q} \bigcup \mathbb{A}(\mathbb{Q})) \backslash \mathbb{B} \backslash \mathbb{D}
$$

with $\mathbb{A}(\mathcal{P})$ and $\mathbb{B}$ as in $(11.60)$ and

$$
\mathbb{D}=\left\{\left(\sigma, r, s, n, \frac{p}{q}\right)|r, s, q \in \mathbb{N}, p, n \in \mathbb{Z},| r-s\left(\frac{p}{q}+2\right) \mid \in\{|n|,|n|+1,|n|+2, \ldots\}\right\} .
$$

Then,

1. $\mathcal{R}_{j, \Lambda, k}$ contains a submodule $\mathcal{C}$ generated from the charged singular vector $\left|C\left(n, j, \frac{p}{q}\right)\right\rangle$; if $n<0$, then $\mathcal{C} \approx \mathcal{M}_{j+n, \frac{p}{q}}$, where $\mathcal{M}_{j+n, \frac{p}{q}}$ is a Verma module with at least two singular vectors; if $n \geq 0, \mathcal{C} \approx \mathcal{M}_{j+n+1+\frac{p}{2 q}, \frac{p}{q} ; 1}$.

2. Any other primitive submodule in $\mathcal{R}_{j, \Lambda, k}$ is isomorphic either to a relaxed Verma module or, depending on whether $n<0$ or $n \geq 0$, to a Verma module or a twisted Verma module with the twist parameter $\theta=1$, respectively. Further,

(a) if $n<0$, each Verma submodule $\mathcal{C}^{\prime} \subset \mathcal{R}_{j, \Lambda, k}$ is a submodule in $\mathcal{C}$ and, moreover, there exists a relaxed Verma submodule $\mathcal{R}^{\prime}$ such that $\mathcal{C}^{\prime}$ is generated from a charged singular vector in $\mathcal{R}^{\prime}$.

(b) if $n \geq 0$, each twisted Verma submodule $\mathcal{C}^{\prime} \subset \mathcal{R}_{j, \Lambda, k}$ is a submodule in $\mathcal{C}$ and, moreover, there exists a relaxed Verma submodule $\mathcal{R}^{\prime}$ such that $\mathcal{C}^{\prime}$ is generated from a charged singular vector in $\mathcal{R}^{\prime}$.

(c) for any relaxed Verma submodule $\mathcal{R}^{\prime} \subset \mathcal{R}_{j, \Lambda, k}$, the intersection $\mathcal{C}^{\prime}=\mathcal{R}^{\prime} \cap \mathcal{C}$ is nonempty. If, then, $|v\rangle$ is the highest-weight vector of $\mathcal{C}^{\prime}$, there exists a vector $\widetilde{|v\rangle} \in$ $\mathcal{R}_{j, \Lambda, k}$, defined as

$$
\widetilde{|v\rangle}= \begin{cases}\left(J_{0}^{+}\right)^{-1}|v\rangle, & n \geq 0, \\ \left(J_{0}^{-}\right)^{-1}|v\rangle, & n<0,\end{cases}
$$

that satisfies relaxed highest-weight conditions and generates the relaxed Verma submodule $\mathcal{R}^{\prime}$.

3. For any primitive submodule $\mathcal{C}^{\prime} \subset \mathcal{C}$, there exists a relaxed Verma submodule $\mathcal{R}^{\prime}$ such that (a) $\mathcal{C}^{\prime} \subset \mathcal{R}^{\prime} \cap \mathcal{C}$ 
(b) $\mathcal{R}^{\prime}$ is generated from a vector $\widetilde{|v\rangle}$ such that the vector

$$
|v\rangle= \begin{cases}J_{0}^{+} \widetilde{|v\rangle}, \quad n \geq 0 \\ J_{0}^{-} \widetilde{|\widetilde{v}\rangle}, \quad n<0\end{cases}
$$

generates a Verma module (if $n<0$ ) or a twisted Verma module (if $n \geq 0$ ) that either coincides with $\mathcal{C}^{\prime}$ or contains $\mathcal{C}^{\prime}$ as a submodule generated from the singular vector $\left|\mathrm{MFF}^{+}(a, 1, k)\right\rangle$ (if $\left.n<0\right)$ or $\left|\mathrm{MFF}^{-, 1}(a, 1, k)\right\rangle$ (if $\left.n \geq 0\right)$, where $a \in \mathbb{N}$.

Whenever $s=0$, we have $\mathcal{R}^{\prime}=\mathcal{R}_{j, \Lambda, k}$.

Thus, the structure of $\mathcal{R}_{j, \Lambda, k}$ is completely determined by the structure of its Verma submodule $\mathcal{C}=\mathcal{M}_{\mathbf{j}^{-}\left(r, s, \frac{p}{q}\right), \frac{p}{q}} \subset \mathcal{R}_{j, \Lambda, k}($ if $n<0)$ or $\mathcal{C}=\mathfrak{M}_{\mathrm{j}^{+}\left(r, s, \frac{p}{q}\right), \frac{p}{q} ; 1} \subset \mathcal{R}_{j, \Lambda, k}($ if $n \geq 0)$.

The case $O_{r c c}$ of the list of possible degenerations is the most involved one, and we describe it in the following Theorem.

Theorem II.18 The highest-weight of the module $\mathcal{R}_{j, \Lambda, k}$ belongs to the set $O_{\text {rcc }}$ of the list of degeneration patterns if and only if

$$
j=j_{\mathrm{cc}}(n, m), \quad \Lambda=\Lambda_{\mathrm{cc}}(n, m), \quad-n, m+1 \in \mathbb{N}, \quad k+2=\frac{r \pm(m-n)}{s}, \quad r, s \in \mathbb{N} .
$$

In this case, the module $\mathcal{R}_{j, \Lambda, k}$ contains a Verma submodule $\mathcal{C}_{-}$generated from the charged singular vector $\left|C\left(n, j_{\mathrm{cc}}(n, m), k\right)\right\rangle$ and a Verma submodule $\mathcal{C}_{+}$(with the twist parameter $\theta=1$ ) generated from the charged singular vector $\left|C\left(m, j_{\mathrm{cc}}(n, m), k\right)\right\rangle$. Each of the modules $\mathcal{C}_{-}$and $\mathcal{C}_{+}$admits at least one singular vector. Moreover, a singular vector $\left|\operatorname{MFF}^{ \pm}(a, b, k)\right\rangle$ exists in the module $\mathcal{C}_{-}$if and only if the singular vector $\left|\mathrm{MFF}^{\mp, 1}(a, b, k)\right\rangle$ with the same $a, b \in \mathbb{N}$ exists in $\mathcal{C}_{+}$.

Any other primitive submodule $\mathcal{N} \subset \mathcal{R}_{j, \Lambda, k}$ is one of the following:

1. $\mathcal{N}$ is a Verma submodule, such that $\mathcal{N} \subset \mathcal{C}_{-}$;

2. $\mathcal{N}$ is a twisted Verma submodule with the twist parameter 1 , such that $\mathcal{N} \subset \mathcal{C}_{+}$;

3. $\mathcal{N}$ is a relaxed Verma module, in which case the intersections $\mathcal{N} \cap \mathcal{C}_{-}=\mathcal{C}_{-}^{\prime}$ and $\mathcal{N} \cap \mathcal{C}_{+}=$ $\mathcal{C}_{+}^{\prime}$ are non-empty. Whenever $\mathcal{C}_{+}^{\prime}$ is generated from a singular vector $\left|\operatorname{MFF}^{ \pm}(a, b, k)\right\rangle$, $\mathcal{C}_{-}^{\prime}$ is generated from singular vector $\left|\operatorname{MFF}^{\mp}(a, b, k)\right\rangle$ with the same $a, b \in \mathbb{N}$ (and the above $k$ ). 
Equivalence between $\widehat{s \ell}(2)$ and $N=2$ representations

Next, we give explicit constructions of singular vectors that generate submodules described in Theorem I1.18:

Theorem II.19 Under the conditions of Theorem II.18,

1. whenever $k+2=\frac{m-n+r}{s}$, the module $\mathcal{R}_{j, \Lambda, k}$ contains a relaxed submodule $\mathcal{R}^{\prime}$ such that its intersections with $\mathcal{C}_{-}$and $\mathcal{C}_{+}$are generated from the following vectors:

$$
\begin{aligned}
|\mathrm{mff}\rangle^{+} & =\mathcal{M F F}^{+}(r, s+1, k)\left(J_{0}^{-}\right)^{-n}\left|j_{\mathrm{cc}}(n, m), \Lambda_{\mathrm{cc}}(n, m), k\right\rangle_{s \ell(2)}, \\
|\mathrm{mff}\rangle^{-, 1} & =\mathcal{M} \mathcal{F} \mathcal{F}^{-, 1}(r, s+1, k)\left(J_{0}^{+}\right)^{m+1}\left|j_{\mathrm{cc}}(n, m), \Lambda_{\mathrm{cc}}(n, m), k\right\rangle_{s \ell(2)} .
\end{aligned}
$$

respectively, and $\mathcal{R}^{\prime}$ itself is generated from any of the states

$$
\left(J_{0}^{-}\right)^{-1}|\mathrm{mff}\rangle^{+} \quad \text { or } \quad\left(J_{0}^{+}\right)^{-1}|\mathrm{mff}\rangle^{-, 1}
$$

The states $|\mathrm{mff}\rangle^{+}$and $|\mathrm{mff}\rangle^{-, 1}$ are charged singular vectors in $\mathcal{R}^{\prime}$; after taking the quotient of $\mathcal{R}^{\prime}$ with respect to $|\mathrm{mff}\rangle^{+}$and $|\mathrm{mff}\rangle^{-, 1}$, the states remaining on the top level furnish an irreducible representation of the Lie algebra sl(2) of dimension $m-n+2 r$;

2. whenever $k+2=\frac{-m+n+r}{s}$ and $2 r-m+n \leq-1$, the module $\mathcal{R}_{j, \Lambda, k}$ contains a relaxed Verma submodule $\mathcal{R}^{\prime}$ such that its intersections with $\mathcal{C}_{-}$and $\mathcal{C}_{+}$are generated from the vectors

$$
\begin{aligned}
|\mathrm{mff}\rangle^{-} & =\mathcal{M F F}^{-}(r, s, k)\left(J_{0}^{-}\right)^{-n}\left|j_{\mathrm{cc}}(n, m), \Lambda_{\mathrm{cc}}(n, m), k\right\rangle_{s \ell(2)} \\
|\mathrm{mff}\rangle^{+, 1} & =\mathcal{M F F}^{+, 1}(r, s, k)\left(J_{0}^{+}\right)^{m+1}\left|j_{\mathrm{cc}}(n, m), \Lambda_{\mathrm{cc}}(n, m), k\right\rangle_{s \ell(2)}
\end{aligned}
$$

respectively, and $\mathcal{R}^{\prime}$ itself is generated from any of the states

$$
\left(J_{0}^{-}\right)^{-1}|\mathrm{mff}\rangle^{-} \quad \text { or } \quad\left(J_{0}^{+}\right)^{-1}|\mathrm{mff}\rangle^{+, 1}
$$

The vectors $|\mathrm{mff}\rangle^{-}$and $|\mathrm{mff}\rangle^{+, 1}$ are charged singular vectors in $\mathcal{R}^{\prime}$; after taking the quotient with respect to $|\mathrm{mff}\rangle^{+}$and $|\mathrm{mff}\rangle^{-, 1}$, the states remaining on the top level of $\mathcal{R}^{\prime}$ furnish an irreducible representation of the Lie algebra $s \ell(2)$ of dimension $m-n-2 r$;

3. whenever $k+2=\frac{-m+n+r}{s}$ and $2 r-m+n \geq 0$, the module $\mathcal{R}_{j, \Lambda, k}$ contains submodules $\mathcal{C}_{-}^{\prime} \subset \mathcal{C}_{-}$and $\mathcal{C}_{+}^{\prime} \subset \mathcal{C}_{+}$that are generated from singular vectors ([I.79) respectively. There does not exist a relaxed Verma submodule $\mathcal{R}^{\prime} \subset \mathcal{R}_{j, \Lambda, k}$ in which either $|\mathrm{mff}\rangle^{-}$or $|\mathrm{mff}\rangle^{+, 1}$ would be a charged singular vector.

Moreover, whenever $2 r-m+n \geq 1$, the states $\left(J_{0}^{+}\right)^{i}|\mathrm{mff}\rangle^{+, 1}$ and $\left(J_{0}^{-}\right)^{2 r-m+n-1-i}|\mathrm{mff}\rangle^{-}$, $i=0, \ldots, 2 r-m+n-1$, satisfy the relaxed highest-weight conditions, are in the same 
grade for each $i$ and are linearly independent. The module $\mathcal{C}_{-}^{\prime}$ contains the singular vector $\left|\mathrm{MFF}^{+}\left(2 r-m+n, 1, \frac{-m+n+r}{s}-2\right)\right\rangle$, while the module $\mathcal{C}_{+}^{\prime}$ contains the singular vector $\left|\mathrm{MFF}^{-, 1}\left(2 r-m+n, 1, \frac{-m+n+r}{s}-2\right)\right\rangle$. After taking the quotients with respect to the submodules generated from these two singular vectors, the states remaining in the top level of the submodule $\mathcal{C}_{-}^{\prime} \cup \mathcal{C}_{+}^{\prime}$ arrange into a direct sum of two irreducible representations of the $s \ell(2)$ Lie algebra of the dimension $2 r-m+n$ each.

Parts 1 and 2 of Theorem 11.19 follow from Lemma [1.16. This can be illustrated as

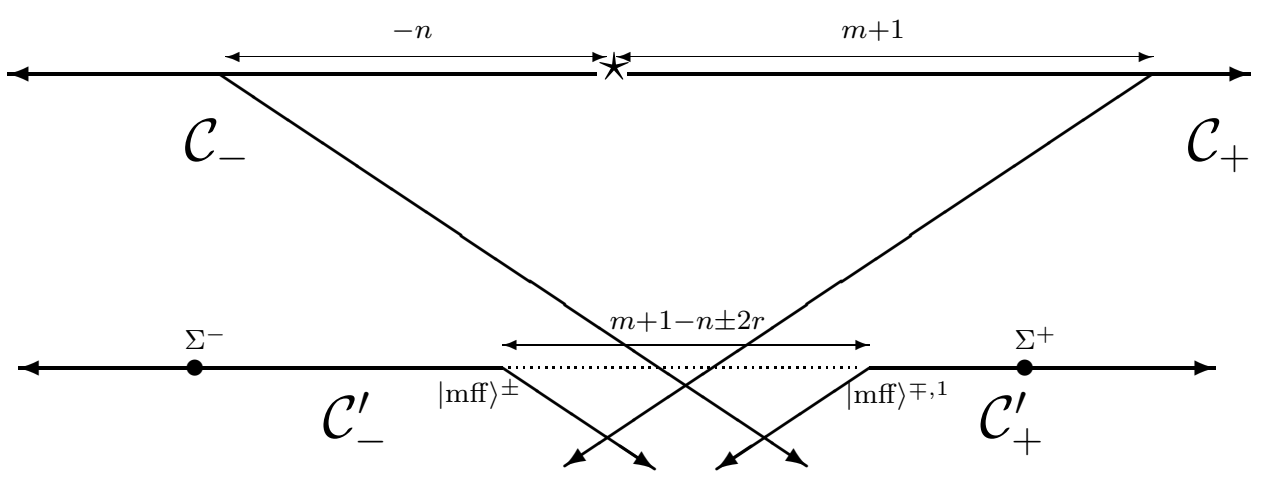

where the upper signs are to be taken in case 1 of the Theorem, and the lower signs, in case 2 .

In case 3 of the Theorem, the appearance of singular vectors $\left|\mathrm{MFF}^{+}\left(2 r-m+n, 1, \frac{n+r-m}{s}-2\right)\right\rangle$ and $\left|\mathrm{MFF}^{-, 1}\left(2 r-m+n, 1, \frac{n+r-m}{s}-2\right)\right\rangle$ in the modules $\mathcal{C}_{-}^{\prime}$ and $\mathcal{C}_{+}^{\prime}$ respectively follows by a direct analysis of the relevant highest-weights. This can be illustrated by the extremal diagram

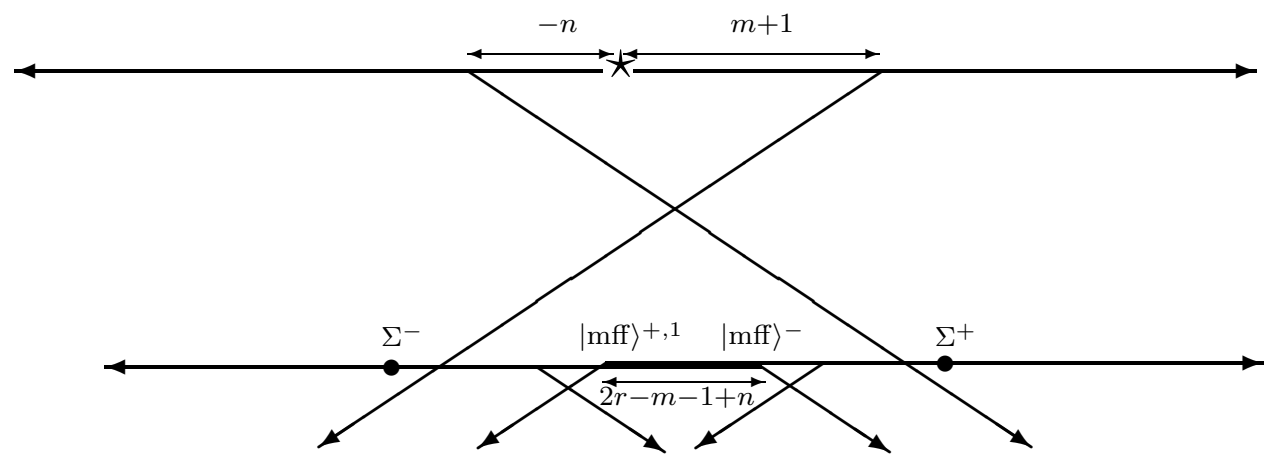

where $|\mathrm{mff}\rangle^{+, 1}$ lies on the upper of the two overlapping lines, and $|\mathrm{mff}\rangle^{-}$, on the lower one.

Remark II.20 As we see from the Theorem, whenever two different singular vectors appear in the same grade, these are necessarily descendants of singular vectors in the corresponding (twisted) Verma submodules. Thus, understanding by the relaxed singular vector proper the entire floor in the extremal diagram, we can say that two different relaxed singular vectors in the same grade never occur. 
This concludes the list of our constructions on the $\widehat{s \ell}(2)$ side. The above formulation was given in such a way as to facilitate comparison with the $N=2$ superconformal algebra; as we will see later, all of the above objects and constructions have appropriate $N=2$ analogues.

\section{II.2 Auxiliaries}

We now introduce two free-field systems that will be needed in Sec. III.

\section{II.2.1 Fermions (ghosts)}

We will need a fermionic system (the so-called ghosts, or a $b c$ system), defined in terms of the operator products as

$$
B(z) C(w)=\frac{1}{z-w}
$$

with the energy-momentum tensor

$$
T^{\mathrm{GH}}=-B \partial C
$$

We use the mode expansions $B(z)=\sum_{n \in \mathbb{Z}} B_{n} z^{-n-1}$ and $C(z)=\sum_{n \in \mathbb{Z}} C_{n} z^{-n}$.

Denote by $\Omega$ the module generated from the vacuum $|0\rangle_{\mathrm{GH}}$ defined by the conditions

$$
C_{\geq 1}|0\rangle_{\mathrm{GH}}=B_{\geq 0}|0\rangle_{\mathrm{GH}}=0
$$

The thus defined vacuum is an $s l_{2}$-invariant state [23]; along with this one, we can choose other highest-weight states, $|\lambda\rangle_{\mathrm{GH}}$, which belong to the same module and are determined by

$$
C_{\geq 1-\lambda}|\lambda\rangle_{\mathrm{GH}}=B_{\geq \lambda}|\lambda\rangle_{\mathrm{GH}}=0
$$

Then, as is easy to see,

$$
\begin{aligned}
(B C)_{0}|\lambda\rangle_{\mathrm{GH}} & =-\lambda|\lambda\rangle_{\mathrm{GH}}, \\
L_{0}^{\mathrm{GH}}|\lambda\rangle_{\mathrm{GH}} & =\frac{1}{2} \lambda(\lambda-1)|\lambda\rangle_{\mathrm{GH}} .
\end{aligned}
$$

The character of $\Omega$ is given by

$$
\chi^{\mathrm{GH}}(z, q)=\operatorname{Tr}_{\Omega}\left(q^{L_{0}^{\mathrm{GH}}} z^{-(B C)_{0}}\right)=\prod_{i=0}^{\infty}\left(1+z q^{i}\right) \prod_{i=1}^{\infty}\left(1+z^{-1} q^{i}\right) .
$$

We can connect the states $|\lambda\rangle_{\mathrm{GH}}$ with different $\lambda$ by means of operators $c(\mu, \nu)$ and $b(\mu, \nu)$ which are the products of fermionic modes

$$
c(\mu, \nu)=\prod_{n=1}^{\nu-\mu+1} C_{\mu+n}, \quad b(\mu, \nu)=\prod_{n=1}^{\nu-\mu+1} B_{\nu+n}, \quad \nu-\mu+1 \in \mathbb{N} .
$$


These $c(\mu, \nu)$ and $b(\mu, \nu)$ map a vector $|\lambda\rangle_{\mathrm{GH}}$ as follows

$$
c(-\lambda-\ell+1,-\lambda):|\lambda\rangle_{\mathrm{GH}} \mapsto|\lambda+\ell\rangle_{\mathrm{GH}}, \quad b(-\lambda-\ell, \lambda-1):|\lambda\rangle_{\mathrm{GH}} \mapsto|\lambda-\ell\rangle_{\mathrm{GH}}, \quad \ell \in \mathbb{N} .
$$

All the necessary properties of the objects $c(\mu, \nu)$ and $b(\mu, \nu)$ can be derived when $\nu-\mu+1$ is a positive integer and then generalized to the case of arbitrary $\mu$ and $\nu$. For example,

$$
c(\mu, \nu-1) c\left(\nu, \nu_{1}\right)=c\left(\mu, \nu_{1}\right), \quad b(\mu, \nu-1) b\left(\nu, \nu_{1}\right)=b\left(\mu, \nu_{1}\right) .
$$

\section{II.2.2 The 'Liouville' scalar}

In this subsection we introduce a 'Liouville' scalar which will be used to invert the KS mapping. This is just a free scalar, called 'Liouville' for the signature of its operator product:

$$
\phi(z) \phi(w)=-\ln (z-w)
$$

We define vertex operators $\psi=e^{\phi}$ and $\psi^{*}=e^{-\phi}$ with the mode expansions

$$
\psi(z)=\sum_{n=-\infty}^{\infty} \frac{\psi_{n}}{z^{n}}, \quad \psi^{*}(z)=\sum_{n=-\infty}^{\infty} \frac{\psi_{n}^{*}}{z^{n-1}},
$$

and

$$
\partial \phi(z)=\sum_{n=-\infty}^{\infty} \frac{\phi_{n}}{z^{n+1}}, \quad \text { with } \quad\left[\phi_{n}, \phi_{m}\right]=-n \delta_{n+m, 0} .
$$

We take the energy-momentum tensor to be

$$
T_{\phi}=-\frac{1}{2} \partial \phi \partial \phi+\frac{1}{2} \partial^{2} \phi
$$

then $\psi$ and $\psi^{*}$ have the dimensions 0 and -1 respectively.

The $\psi$ and $\psi^{*}$ operators are naturally represented as intertwining operators on a direct sum

$$
\Xi=\bigoplus_{n \in \mathbb{Z}} \mathcal{H}_{n}
$$

where $\mathcal{H}_{n}$ is a Verma module over the Heisenberg algebra ([1.94) with the highest-weight vector $|n\rangle_{\phi}$

$$
\begin{aligned}
& \phi_{m}|n\rangle_{\phi}=0, \quad m \geq 1, \quad \psi_{m}|n\rangle_{\phi}=0, \quad m \geq n+1, \quad \psi_{m}^{*}|n\rangle_{\phi}=0, \quad m \geq-n+2, \\
& \phi_{0}|n\rangle_{\phi}=-n|n\rangle_{\phi} .
\end{aligned}
$$

Note also that the highest-weight vectors $|n\rangle_{\phi}$ can be realized as the operators $|n\rangle_{\phi} \leadsto e^{n \phi}(0)$. 


\section{II.3 $N=2$}

In the description of our second main ingredient, the $N=2$ algebra, we follow refs. [9, 10], particularly as regards the singular vectors. Other (non-exhaustive) references on the $N=2$ superconformal algebra are [3, 12, 15, 16, EHY].

\section{II.3.1 The $N=2$ superconformal algebra}

The $N=2$ superconformal algebra is spanned by Virasoro generators $L_{m}$, two fermionic fields $G_{r}^{ \pm}$, and a $U(1)$ current $H_{n}$ (and a central element C). The nonvanishing commutation relations are:

$$
\begin{array}{ll}
{\left[L_{n}, L_{m}\right]=(n-m) L_{n+m}+\frac{\mathrm{C}}{12}\left(n^{3}-n\right) \delta_{n+m},} & {\left[H_{n}, H_{m}\right]=\frac{\mathrm{C}}{3} n \delta_{n+m},} \\
{\left[L_{n}, H_{m}\right]=-m H_{n+m}, \quad\left[L_{n}, G_{r}^{ \pm}\right]=\left(\frac{1}{2} n-r\right) G_{n+r}^{ \pm},} & {\left[H_{n}, G_{r}^{ \pm}\right]= \pm G_{n+r}^{ \pm},} \\
\left\{G_{r}^{+}, G_{s}^{-}\right\}=2 L_{r+s}+(r-s) H_{r+s}+\frac{\mathrm{C}}{3}\left(r^{2}-\frac{1}{4}\right) \delta_{r+s}, & n, m \in \mathbb{Z}, \quad r, s \in \mathbb{Z}+\frac{1}{2} .
\end{array}
$$

We find it more convenient to work with the version of this algebra obtained by redefining the generators as

$$
L_{n}=\left.L_{n}\right|_{(\mathrm{II} .98)}+\frac{1}{2}(n+1) H_{n}, \quad G_{r}=G_{r+\frac{1}{2}}^{+}, \quad Q_{r}=G_{r-\frac{1}{2}}^{-} .
$$

This redefinition affects two things: the modding of the fermions and the choice of the Virasoro generators. The latter is due to the freedom of adding a derivative of the $U(1)$ current to the energy-momentum tensor, and is nothing but a change of basis in the algebra. As to the different moddings, they label different members of a family of isomorphic algebras related by the spectral flow [15, 16] (see Eq. ([1.106) below). Thus any statement found for one of these algebras does immediately translate to any other one. In order to be specific in our constructions below, we need to choose the concrete representative from that family as well as the modding of the generators. These will be

$$
\begin{array}{llll}
{\left[L_{m}, L_{n}\right]} & =(m-n) L_{m+n}, & {\left[H_{m}, H_{n}\right]=\frac{C}{3} m \delta_{m+n, 0},} & \\
{\left[L_{m}, G_{n}\right]=(m-n) G_{m+n},} & {\left[H_{m}, G_{n}\right]=G_{m+n},} & \\
{\left[L_{m}, Q_{n}\right]=-n Q_{m+n},} & {\left[H_{m}, Q_{n}\right]=-Q_{m+n}, \quad m, n \in \mathbb{Z} .} \\
{\left[L_{m}, H_{n}\right]=-n H_{m+n}+\frac{c}{6}\left(m^{2}+m\right) \delta_{m+n, 0},} & & \\
\left\{G_{m}, Q_{n}\right\} & =2 L_{m+n}-2 n H_{m+n}+\frac{c}{3}\left(m^{2}+m\right) \delta_{m+n, 0},
\end{array}
$$

Denote this algebra as $\mathcal{A}$. Its generators $L_{m}, Q_{m}, H_{m}$, and $G_{m}$ will be called the Virasoro generators, the 'BRST' current, f the $U(1)$ current, and the spin-2 fermionic current respectively.

\footnotetext{
${ }^{6} Q(z)$ is the BRST current when the algebra ([I.100) is realized on the bosonic string world-sheet 24, 25.
} 
As a Lie superalgebra, $\mathcal{A}$ is generated by the following set of elements:

$$
\begin{array}{lll}
e_{1}=\frac{1}{2} Q_{0}, & e_{2}=G_{1}, & e_{3}=H_{1}, \\
h_{1}=L_{0}, & h_{2}=L_{0}+H_{0}+\frac{\mathrm{C}}{3}, & h_{3}=\frac{\mathrm{C}}{3}, \\
f_{1}=G_{0}, & f_{2}=\frac{1}{2} Q_{-1}, & f_{3}=H_{-1} .
\end{array}
$$

The elements $h_{i}, i=1,2,3$ generate the Cartan subalgebra $\mathfrak{h}$.

Now we define the topological[ Verma modules over the $N=2$ algebra; the highest-weight vector in any such module satisfies the annihilation and eigenvalue conditions

$$
\begin{aligned}
Q_{\geq 0}|h, t\rangle_{\mathrm{top}} & =G_{\geq 0}|h, t\rangle_{\mathrm{top}}=L_{\geq 1}|h, t\rangle_{\mathrm{top}}=H_{\geq 1}|h, t\rangle_{\mathrm{top}}=0 \\
H_{0}|h, t\rangle_{\mathrm{top}} & =h|h, t\rangle_{\mathrm{top}}, \quad L_{0}|h, t\rangle_{\mathrm{top}}=0, \quad \mathrm{C}|h, t\rangle_{\mathrm{top}}=\frac{3(t-2)}{t}|h, t\rangle_{\mathrm{top}}
\end{aligned}
$$

(the last equation being simply a parametrization of the central charge in terms of a new parameter, $t$; later this parameter will be identified with $k+2$, where $k$ is the $\widehat{s \ell}(2)$ level). Accordingly, the topological Verma module $\mathcal{V}_{h, t}$ is freely generated by

$$
L_{-m}, m \in \mathbb{N}, \quad H_{-m}, m \in \mathbb{N}, \quad Q_{-m}, m \in \mathbb{N}, \quad G_{-m}, m \in \mathbb{N}
$$

from the topological highest-weight vector $|h, t\rangle_{\text {top }}$.

The extremal diagram of a topological Verma module is of the form

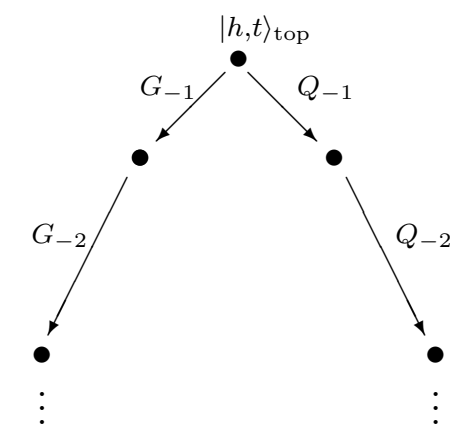

where the bigrading in the plane of the diagram is by (charge, level).

\section{II.3.2 Automorphisms of the $N=2$ algebra}

Spectral flow transform. When applied to the generators of the algebra $\mathcal{A}$, Eqs. (II.100), the spectral flow transformation $\mathcal{U}_{\theta}$ acts as

$$
\begin{array}{rlrl}
L_{n} & \mapsto L_{n}+\theta H_{n}+\frac{\mathrm{C}}{6}\left(\theta^{2}+\theta\right) \delta_{n, 0}, & H_{n} & \mapsto H_{n}+\frac{\mathrm{C}}{3} \theta \delta_{n, 0}, \\
Q_{n} & \mapsto Q_{n-\theta}, & G_{n} \mapsto G_{n+\theta} .
\end{array}
$$

\footnotetext{
${ }^{7}$ The name is inherited from the non-critical bosonic string, where matter vertices can be dressed into $N=2$ primaries that satisfy the highest-weight conditions (II.102); in that context, the algebra (II.100) is viewed as a topological algebra.
} 
This gives an isomorphic algebra $\mathcal{A}_{\theta}$, whose generators $L_{n}^{\theta}, Q_{n}^{\theta}, H_{n}^{\theta}$ and $G_{n}^{\theta}$ can be taken as the RHSs of (II.106). For $\theta \in \mathbb{Z}$, the spectral flow is an automorphism of the algebra $\mathcal{A}$. For arbitrary $\theta$, we obtain a family of algebras such that any two of its members, $\mathcal{A}_{\theta_{1}}$ and $\mathcal{A}_{\theta_{2}}$, are related by an isomorphism of the type of ([1.106) with $\theta=\theta_{1}-\theta_{2}$. This family includes the Neveu-Schwarz and Ramond $N=2$ algebras, as well as the algebras in which the fermion modes range over $\pm \theta+\mathbb{Z}, \theta \in \mathbb{C}$.

The involutive automorphism. The $N=2$ algebra has the involutive automorphism

$$
\begin{aligned}
& G_{n} \rightarrow Q_{n+1}, \quad Q_{n} \rightarrow G_{n-1}, \\
& H_{n} \rightarrow-H_{n}, \quad L_{n} \rightarrow L_{n}-(n+1) H_{n} .
\end{aligned}
$$

Together with automorphisms (II.106) for $\theta \in \mathbb{Z}$, transformations ([I.107) span the group $\mathbb{Z}_{2} \times \mathbb{Z}$ isomorphic to the group generated by the canonical involution and the spectral flow of the affine $s \ell(2)$ algebra.

\section{II.3.3 Twisted topological Verma modules}

We now define the twisted topological Verma modules $\mathfrak{V}_{h, t ; \theta}$. This will be a module over the algebra $\mathcal{A}_{\theta}$ (see $(\overline{[1.106})$ ), with the twisted topological highest-weight vector $|h, t ; \theta\rangle_{\text {top }}$ defined by

$$
\begin{aligned}
& L_{m}|h, t ; \theta\rangle_{\text {top }}=0, \quad m \geq 1, \quad Q_{\lambda}|h, t ; \theta\rangle_{\text {top }}=0, \quad \lambda \in-\theta+\mathbb{N}_{0} \\
& H_{m}|h, t ; \theta\rangle_{\text {top }}=0, \quad m \geq 1, \quad G_{\nu}|h, t ; \theta\rangle_{\text {top }}=0, \quad \nu \in \theta+\mathbb{N}_{0} \\
& \theta \in \mathbb{Z}
\end{aligned}
$$

in addition, the 'Cartan' generators act on the state $|h, t ; \theta\rangle_{\text {top }}$ as

$$
\begin{aligned}
\left(H_{0}+\frac{\mathrm{C}}{3} \theta\right)|h, t ; \theta\rangle_{\text {top }} & =h|h, t ; \theta\rangle_{\text {top }}, \\
\left(L_{0}+\theta H_{0}+\frac{\mathrm{C}}{6}\left(\theta^{2}+\theta\right)\right)|h, t ; \theta\rangle_{\mathrm{top}} & =0, \\
\mathrm{C}|h, t ; \theta\rangle_{\mathrm{top}} & =\frac{3(t-2)}{t}|h, t ; \theta\rangle_{\mathrm{top}} .
\end{aligned}
$$

The $\theta=0$ case of ([I.108) describes the 'ordinary' topological Verma modules introduced above.

Next, we need the concept of terminating fermionic chains.

Definition II.21 Let $F$ denote either $Q$ or $G$ (the fermionic $N=2$ generators), and $|X\rangle$ be an element of a module over the $N=2$ algebra. Fix also an integer $n$. We say that the fermionic $F$-chain terminates on $|X\rangle$, and write $\ldots F_{n-3} F_{n-2} F_{n-1} F_{n}|X\rangle=0$ if

$$
\exists N \in \mathbb{Z}, \quad N \leq n: F_{N} F_{N+1} \ldots F_{n}|X\rangle=0 .
$$


Equivalence between $\widehat{s \ell}(2)$ and $N=2$ representations

Now we are in a position to give the following

Definition II.22 An $N=2$ module $\mathfrak{U}$ is an object of the topological $N=2$ category $\mathcal{T O P}$ whenever the following conditions are satisfied:

1. $H_{0}$ and $L_{0}$ are diagonalizable operators, i.e. $\mathfrak{U}$ can be decomposed into a direct sum of intersections of $H_{0}$ - and $L_{0}$ - eigenspaces;

2. The action with all $H_{p}, p \in \mathbb{N}$, and $L_{p}, p \in \mathbb{N}$, on any element $|X\rangle \in \mathfrak{U}$ spans a finitedimensional space;

3. for any element $|X\rangle$ of $\mathfrak{U}$,

$$
\begin{array}{lll}
\forall n \in \mathbb{Z} & \text { either } & \ldots Q_{n-3} Q_{n-2} Q_{n-1} Q_{n}|X\rangle=0 \\
& \text { or } & \ldots G_{-n-4} G_{-n-3} G_{-n-2} G_{-n-1}|X\rangle=0
\end{array}
$$

Morphisms are standard homomorphisms between $N=2$-modules.

Condition ([I.110) will be called the condition of terminating fermionic chains.

Note finally that the characters of twisted topological Verma modules are given by

$$
\begin{aligned}
\chi_{h, \theta}^{N=2}(z, q) & =\operatorname{Tr}_{\mathfrak{V}_{h, t ; \theta}\left(q^{L_{0}} z^{H_{0}}\right)} \\
& =q^{-\theta h+\mathrm{C}\left(\theta^{2}-\theta\right) / 6} z^{h-\mathrm{C} \theta / 3} \frac{\prod_{i=-\theta+1}^{\infty}\left(1+z q^{i}\right) \prod_{i=\theta+1}^{\infty}\left(1+z^{-1} q^{i}\right)}{\prod_{i=1}^{\infty}\left(1-q^{i}\right)^{2}}
\end{aligned}
$$

\section{II.3.4 Topological $N=2$ singular vectors}

Here, we describe singular vectors in the topological Verma modules introduced above; later, we will see that these are isomorphic to the $\widehat{s \ell}(2)$ singular vectors, as suggested in [7]. We introduce two operators $g(\mu, \nu)$ and $q(\mu, \nu)$, with $\mu, \nu \in \mathbb{C}$, that represent the action of two ' $N=2$ Weyl group' generators $r_{\alpha_{1}}$ and $r_{\alpha_{2}}$ when $\mu$ and $\nu$ are special (see [9, 10] for the details): the operators $g(\mu, \nu)$ and $q(\mu, \nu)$ act on the plane $t=$ const as

$$
\begin{aligned}
& g(h t+\theta-1, \theta-1):|h, t ; \theta\rangle_{\mathrm{top}} \mapsto\left|\frac{2}{t}-h, t ; h t+\theta-1\right\rangle_{\mathrm{top}}, \\
& q(-(h+1) t-\theta+1,-\theta-1):|h, t ; \theta\rangle_{\mathrm{top}} \mapsto\left|\frac{2}{t}-2-h, t ;(h+1) t+\theta-1\right\rangle_{\mathrm{top}} .
\end{aligned}
$$

The action of operators $g(a, b)$ and $q(a, b)$ on the highest-weight vectors can be extended to the topological Verma module generated from these highest-weight vectors 10. This allows us to construct singular vectors in topological Verma modules. 


\section{Theorem II.23}

I. A topological singular vector exists in the topological Verma module $\mathcal{V}_{h, t}$ if and only if either $h=\mathrm{h}^{+}(r, s, t)$ or $h=\mathrm{h}^{-}(r, s, t)$, where

$$
\begin{aligned}
& \mathrm{h}^{+}(r, s, t)=-\frac{r-1}{t}+s-1, \\
& \mathrm{~h}^{-}(r, s, t)=\frac{r+1}{t}-s,
\end{aligned} \quad r, s \in \mathbb{N} .
$$

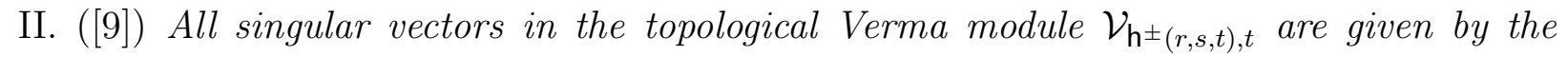
explicit construction:

$$
\begin{gathered}
|E(r, s, t)\rangle^{+}=g(-r,(s-1) t-1) q((1-s) t, r-1-t) \ldots \\
\cdot g((s-2) t-r, t-1) q(-t, r-1-(s-1) t) \\
\cdot g((s-1) t-r,-1)\left|\mathrm{h}^{+}(r, s, t), t\right\rangle_{\mathrm{top}}, \\
|E(r, s, t)\rangle^{-}=q(-r,(s-1) t-1) g((1-s) t, r-t-1) \ldots \\
\cdot q((s-2) t-r, t-1) g(-t, r-1-(s-1) t) \\
\cdot q((s-1) t-r,-1)\left|\mathrm{h}^{-}(r, s, t), t\right\rangle_{\mathrm{top}}
\end{gathered}
$$

where $r, s \in \mathbb{N}$ and the factors in the first two lines of each formula are

$$
g(-r-t-m t+s t,-1+m t) q(-m t, r-1+m t-s t), \quad s-1 \geq m \geq 1
$$

and

$$
q(-r-t-m t+s t,-1+m t) g(-m t, r-1+m t-s t), \quad s-1 \geq m \geq 1
$$

respectively.

Part I can be deduced from [12]. Singular vectors (ㅍ.115), ([I.114) satisfy highest-weight conditions (ㅍ.108) with $\theta=\mp r$ respectively.

\section{II.3.5 Massive $N=2$ Verma modules}

We now introduce $N=2$ Verma modules of a different type, in which the highest-weight states satisfy the following annihilation and eigenvalue conditions:

$$
\begin{aligned}
Q_{\geq 1}|h, \ell, t\rangle & =G_{\geq 0}|h, \ell, t\rangle=L_{\geq 1}|h, \ell, t\rangle=H_{\geq 1}|h, \ell, t\rangle=0 \\
H_{0}|h, \ell, t\rangle & =h|h, \ell, t\rangle, \quad L_{0}|h, \ell, t\rangle=\ell|h, \ell, t\rangle .
\end{aligned}
$$


The module freely generated from $|h, \ell, t\rangle$ by the remaining $N=2$ generators will be denoted as $U_{h, \ell, t}$. Because of the property to have a dimension $\ell$ generally different from zero, $|h, \ell, t\rangle$ are called the massive highest-weight states and $U_{h, \ell, t}$, accordingly, the massive $N=2$ modules.

The twists of massive Verma modules are introduced by imposing the highest-weight conditions (as before, $\mathrm{c}=\frac{3(t-2)}{t}$ )

$$
\begin{aligned}
& L_{m}|h, \ell, t ; \theta\rangle=0, \quad m \geq 1, \quad Q_{\lambda}|h, \ell, t ; \theta\rangle=0, \quad \lambda \in-\theta+\mathbb{N} \\
& H_{m}|h, \ell, t ; \theta\rangle=0, \quad m \geq 1, \quad G_{\nu}|h, \ell, t ; \theta\rangle=0, \quad \nu=\theta+\mathbb{N}_{0} \\
& \left(H_{0}+\frac{c}{3} \theta\right)|h, \ell, t ; \theta\rangle=h|h, \ell, t ; \theta\rangle, \quad\left(L_{0}+\theta H_{0}+\frac{c}{6}\left(\theta^{2}+\theta\right)\right)|h, \ell, t ; \theta\rangle=\ell|h, \ell, t ; \theta\rangle,
\end{aligned}
$$

Extremal diagrams of massive Verma modules have the following 'fat' form for $\theta=0$ (see [10] for the details)

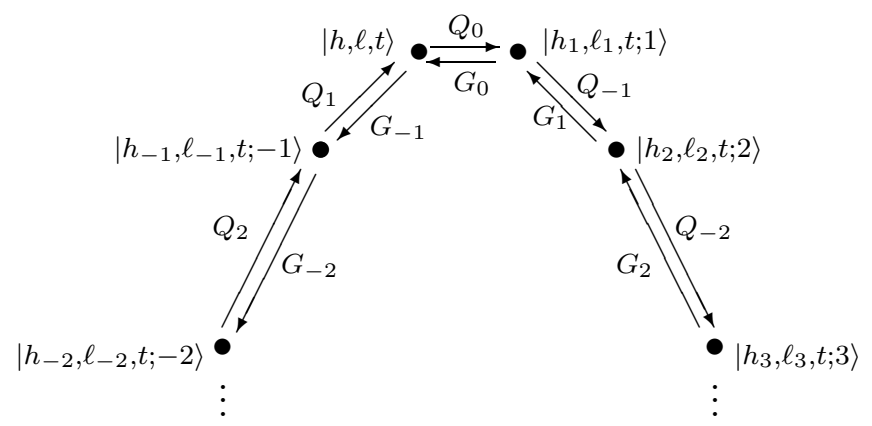

The states in the diagram are freely generated from $|h, \ell, t\rangle$ by the $G_{-1} G_{-2} \ldots$ and $Q_{0} Q_{-1} \ldots$ arrows in accordance with the definition of massive Verma modules. The arrows mapping back towards $|h, \ell, t\rangle$ are described in the following lemma:

Lemma II.24 The mappings

$$
\begin{aligned}
\left|h_{\theta}, \ell_{\theta}, t ; \theta\right\rangle & \mapsto\left|h_{\theta+1}, \ell_{\theta+1}, t ; \theta+1\right\rangle, \quad \theta<0, \\
\left|h_{\theta}, \ell_{\theta}, t ; \theta\right\rangle & \mapsto\left|h_{\theta-1}, \ell_{\theta-1}, t ; \theta-1\right\rangle, \quad \theta>0
\end{aligned}
$$

are implemented by $Q_{-\theta}$ and $G_{\theta-1}$ respectively:

$$
\begin{aligned}
Q_{-\theta}|h, \ell, t ; \theta\rangle & =2 \ell\left|h-\frac{2}{t}, \ell+h-\frac{2}{t}, t ; \theta+1\right\rangle, \quad \theta<0, \\
G_{\theta-1}|h, \ell, t ; \theta\rangle & =2(\ell-h)\left|h+\frac{2}{t}, \ell-h, t ; \theta-1\right\rangle, \quad \theta>0
\end{aligned}
$$

It is worth mentioning that, while $|h, \ell, t\rangle$ in (II.121) satisfies the untwisted highest-weight conditions $\left(\theta=0\right.$ in $\left(\left[\frac{\Pi 120}{)}\right)\right.$, the other states in the diagram satisfy the twisted massive highest-weight conditions (III.120) with $\theta$ running over all integers (from $-\infty$ to $+\infty$ as one moves from left to right). 
It follows from (II.123) that, as soon as one of the factors on the right-hand sides vanishes, the respective state would satisfy the twisted topological highest-weight conditions (III.102). At these 'topological points', the extremal diagram branches, e.g.,

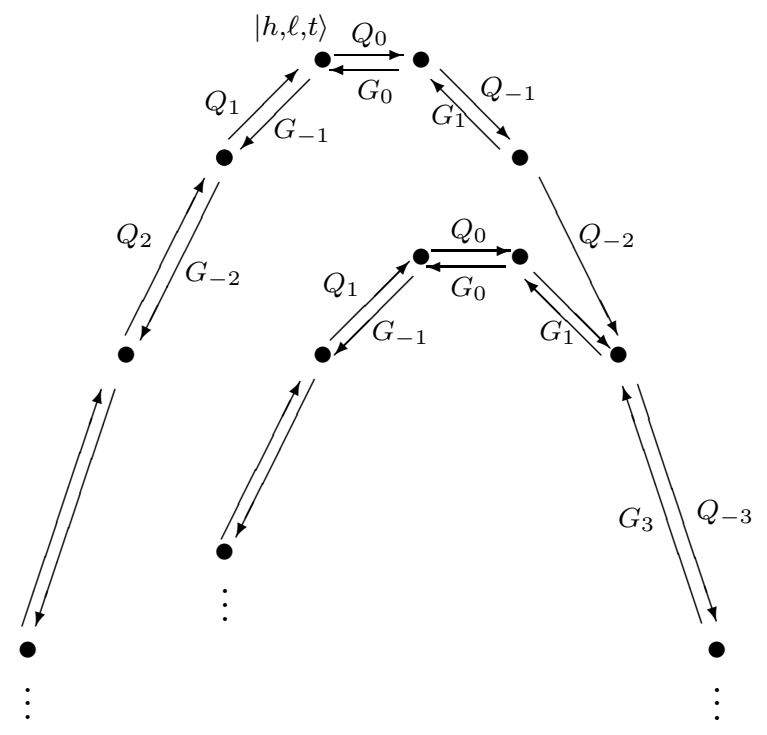

The inner diagram in (II.124) corresponds to an $N=2$ subrepresentation, which is identified with a singular vector. By iteratively applying formulas ([1.123), we arrive at

Theorem II.25 A massive Verma module $U_{h, \ell, t}$ contains a twisted topological Verma submodule if and only if $\ell=\mathrm{I}_{\mathrm{ch}}(r, h, t)$, where

$$
\mathrm{I}_{\mathrm{ch}}(r, h, t)=r\left(h+\frac{r-1}{t}\right), \quad r \in \mathbb{Z}
$$

The corresponding singular vector reads

$$
|E(r, h, t)\rangle_{\mathrm{ch}}= \begin{cases}Q_{r} \ldots Q_{0}\left|h, \mathrm{I}_{\mathrm{ch}}(r, h, t), t\right\rangle & r \leq 0, \\ G_{-r} \ldots G_{-1}\left|h, \mathrm{I}_{\mathrm{ch}}(r, h, t), t\right\rangle, & r \geq 1 .\end{cases}
$$

It satisfies twisted topological highest-weight conditions (11.102) with $\theta=-r$.

(these are singular vectors of the 'charged' series from [12]).

The remaining singular vectors in massive Verma modules can be constructed as follows (we only give the 'untwisted' case, that of twisted massive modules follows by applying the spectral flow transform). As follows from the analysis of the Kač determinant [12], the necessary condition for a massive singular vector to exist in the module $\mathcal{U}_{h, \ell, t}$ is $\ell=\mathrm{I}(r, s, h, t)$, where

$$
\mathrm{I}(r, s, h, t)=\frac{1}{2} h-\frac{1}{2} r s-\frac{1}{4 t}+\frac{r^{2}}{4 t}-\frac{1}{4} h^{2} t+\frac{1}{4} s^{2} t, \quad r, s \in \mathbb{N} .
$$


Equivalence between $\widehat{s \ell}(2)$ and $N=2$ representations

The necessary and sufficient condition as well as explicit formulae for the singular vectors are given in the following

\section{Theorem II.26}

I. ([14]) For $\mathrm{c} \neq 3$, a massive singular vector in $\mathcal{U}_{h, \ell, t}$ can be constructed if and only if $\ell=\mathrm{I}(r, s, h, t)$, where $(r, s, h, t) \in(\mathbb{N} \times \mathbb{N} \times \mathbb{C} \times \mathbb{C}) \backslash \mathbb{X}$ with

$$
\mathbb{X}=\left\{(r, s, h, t) \mid s t-r=m-n, h t-1=-m-n, r+s t=0, \quad n \in \mathbb{N}, m \in-\mathbb{N}_{0}\right\}
$$

II. (10]) The massive singular vectors in $\mathcal{U}_{h, \mathrm{I}(r, s, h, t), t}$ are of the form

$$
\begin{array}{r}
|S(r, s, h, t)\rangle^{-}=g\left(-r s, r+\theta^{-}(r, s, h, t)-1\right) \mathcal{E}^{-, \theta^{-}(r, s, h, t)}(r, s, t) \cdot \\
\cdot g\left(\theta^{-}(r, s, h, t),-1\right)|h, \ell(r, s, h, t), t\rangle, \\
|S(r, s, h, t)\rangle^{+}=q\left(1-r s, r-\theta^{+}(r, s, h, t)-1\right) \mathcal{E}^{+, \theta^{+}(r, s, h, t)}(r, s, t) \cdot \\
\cdot q\left(-\theta^{+}(r, s, h, t), 0\right)|h, \ell(r, s, h, t), t\rangle,
\end{array}
$$

where

$$
\begin{aligned}
& \theta^{-}(r, s, h, t)=\frac{t}{2}\left(h-\mathrm{h}^{-}(r, s, t)\right), \\
& \theta^{+}(r, s, h, t)=\frac{t}{2}\left(h-1-\mathrm{h}^{+}(r, s, t)\right)
\end{aligned}
$$

and $\mathcal{E}^{ \pm, \theta}(r, s, t)$ denotes the spectral flow transform (II.106) of the topological singular vector operator $\mathcal{E}^{ \pm}(r, s, t)$. The two expressions ([1.129) in the general position belong to the same extremal diagram and generate the same massive submodule.

This construction translates in the obvious way to the twisted case.

Degenerate cases corresponding to simultaneous occurrence of several $N=2$ singular vectors can be described similarly to the $\widehat{s \ell}(2)$ case. An essential point is to consider on equal footing all states from each extremal diagram. Restricting oneself to only top-level representatives of extremal diagrams leads to additional complications in describing the structure of $N=2$ modules. In a number of degenerate cases, the entire $N=2$ submodule is not generated by the top-level representative of the extremal diagram of the submodule. This happens already in the case where a charged singular vector $|E(n, h, t)\rangle_{\mathrm{ch}}$ (see Theorem [I.25) and a massive singular vector labelled by two integers $r$ and $s$ coexist in a massive $N=2$ module and $r>n$. Describing this case only in terms of top-level representatives of each of the singular vectors would lead one to assume that subsingular vectors exist in the module, since the chosen representative of the singular vector does not generate the entire submodule. 
Let us describe in more detail the case where two charged singular vectors coexist with a massive singular vector. This is directly analogous to the case considered in Part 3 of Theorem 【1.19:

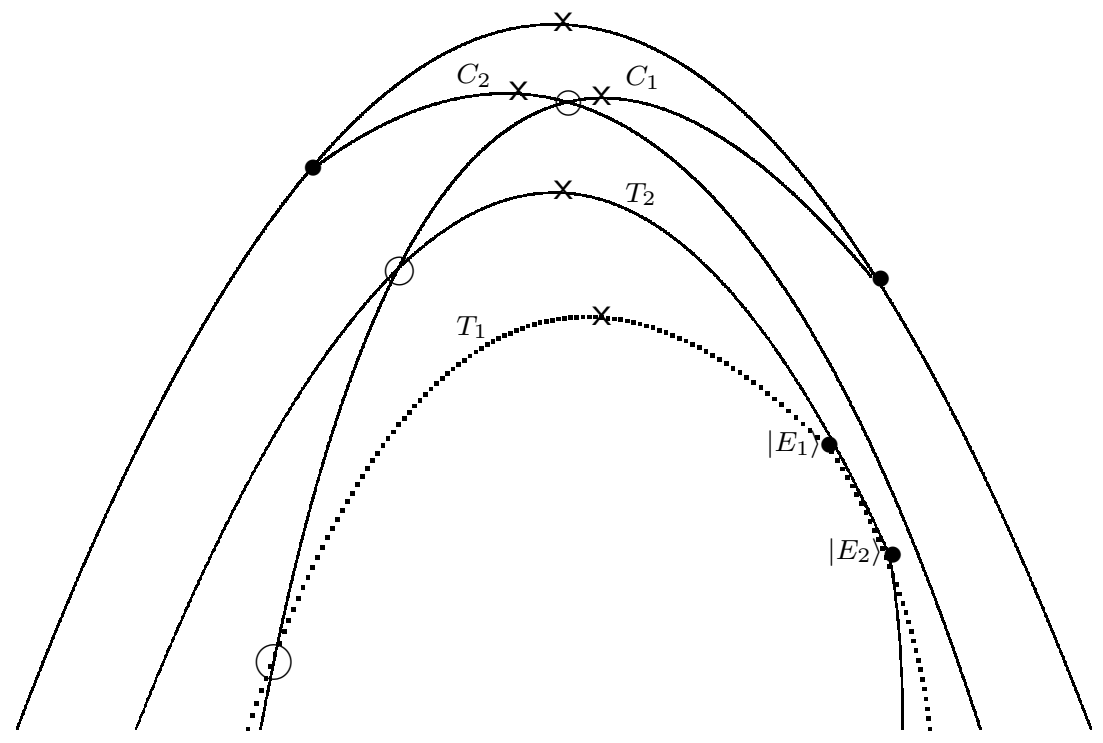

In this extremal diagram, the crosses denote the top-level representatives, the filled dots are twisted topological highest-weight states, and circles indicate that different curves do not intersect, i.e. correspond to linearly independent states at the overlapping points. $C_{1}$ and $C_{2}$ are extremal diagrams of submodules built on the charged singular vectors, while $T_{1}$ and $T_{2}$ are topological submodules in the modules corresponding to $C_{1}$ and $C_{2}$ respectively. Submodules $T_{1}$ and $T_{2}$ are generated by topological singular vectors $\left|E_{1}\right\rangle$ and $\left|E_{2}\right\rangle$ respectively. The pairs of states lying on the section of the $T_{1}$ and $T_{2}$ curves between $\left|E_{1}\right\rangle$ and $\left|E_{2}\right\rangle$ give linearly independent singular vectors in the same grade. For the values of the parameters assumed in the diagram, choosing the top-level representative of $T_{1}$ and $T_{2}$ would conceal the fact that there exist linearly independent singular vectors in the same grade. By an analysis parallel to the one of Sect. [1.1.6, two linearly independent singular vectors in the same grade exist whenever $m-n \leq 2 r$, where $m \geq 1$ and $n \leq-1$ label the two charged singular vectors and $r$ is the first from the pair of integers $(r, s)$ that label the massive singular vector. The top-level representatives would be inside the overlapping section of the two curves only when both $m$ and $|n|$ are $\leq r$; however these restrictions have nothing to do with the structure of submodules that applies whenever $m+|n| \leq 2 r$, and is an artifact of the choice of top-level representatives. Let us note also that in the case where two linearly independent singular vectors exist in the same 
grade, each of these singular vectors belongs to a topological submodule; this is completely similar to the $\widehat{s \ell}(2)$ case discussed above (see Part 3 of Theorem [1.19). For more details and a systematic account of degenerations of $N=2$ Verma modules, we refer the reader to [14].

The above condition for the fermionic chains to terminate is not satisfied for the (twisted) massive Verma modules. Instead, a fermionic chain terminates whenever there are two or more arrows along the same line, e.g.

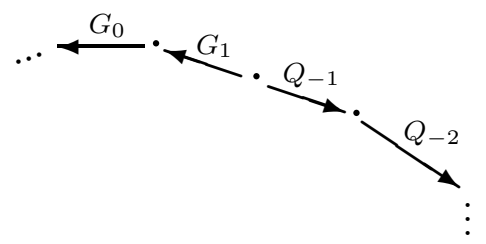

which is formalized in the definition of the category of (twisted) massive highest-weight type modules:

Definition II.27 An $N=2$ module $\mathfrak{W}$ is an object of the category $\mathcal{M H W}$ whenever

1. $H_{0}$ and $L_{0}$ are diagonalizable operators, i.e. $\mathfrak{U}$ can be decomposed into a direct sum of intersections of $H_{0}-L_{0}$-eigenspaces;

2. the action with all $H_{p}, p \in \mathbb{N}$, and $L_{p}, p \in \mathbb{N}$, on any element $|X\rangle \in \mathfrak{U}$ spans a finitedimensional space;

3. for any element $|X\rangle$ of $\mathfrak{U}$,

$$
\begin{array}{lll}
\forall n \in \mathbb{Z} & \text { either } & \ldots Q_{n-3} Q_{n-2} Q_{n-1} Q_{n}|X\rangle=0 \\
& \text { or } & \ldots G_{-n-3} G_{-n-2} G_{-n-1} G_{-n}|X\rangle=0
\end{array}
$$

Morphisms are standard homomorphisms between $N=2$-modules.

\section{Kazama-Suzuki and the related mappings}

\section{III.1 The Kazama-Suzuki mapping}

We use the simplest KS construction associated with the coset $\widehat{s \ell}(2)_{k} \oplus u(1) / u(1)$. The KS 'numerator' $u(1)$ algebra fermionizes into a couple of spin-1 ghosts, denoted in the following as $B C$, which allows us to build up the $N=2$ algebra generators in the standard way [19, 20]: The odd generators $Q$ and $G$ are given by

$$
Q=C J^{+}, \quad G=\frac{2}{k+2} B J^{-},
$$


Equivalence between $\widehat{s \ell}(2)$ and $N=2$ representations

while the $U(1)$ current and the energy-momentum tensor of the $N=2$ algebra take the form

$$
\begin{gathered}
H=\frac{k}{k+2} B C-\frac{2}{k+2} J^{0}, \\
T=\frac{1}{k+2}\left(J^{+} J^{-}\right)-\frac{k}{k+2} B \partial C-\frac{2}{k+2} B C J^{0} .
\end{gathered}
$$

Lemma III.1 The modes of currents (III.1), (III.2), and ([II.3) satisfy the algebra ([I.100). The eigenvalue of the central element in each representation is expressed through the eigenvalue of the $\widehat{s \ell}(2)$ central element as

$$
\mathrm{c}=\frac{3 k}{k+2} .
$$

Thus, Eqs. ([II.1), ([11.2), and ([11.3) determine a mapping

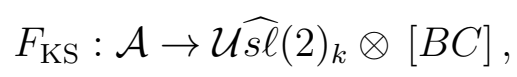

where $\mathcal{A}$ is the $N=2$ algebra ([1.100) and $\mathcal{U}$ denotes the universal enveloping (and $[B C]$ is the free fermion theory).

The KS mapping does also produce a bosonic current

$$
I^{+}=\sqrt{\frac{2}{k+2}}\left(B C+J^{0}\right)
$$

whose modes commute with the $N=2$ generators (III.1)-([II.3). Expanding as $I^{+}(z)=$ $\sum_{n \in \mathbb{Z}} I_{n}^{+} z^{-n-1}$, we get a Heisenberg algebra

$$
\left[I_{m}^{+}, I_{n}^{+}\right]=m \delta_{m+n, 0} .
$$

The energy-momentum tensor of this $U(1)$ current is introduced as

$$
T^{+}=\frac{1}{2}\left(I^{+}\right)^{2}-\frac{1}{\sqrt{2(k+2)}} \partial I^{+} .
$$

We define a module $\mathcal{H}_{p}^{+}$over the Heisenberg algebra by the highest-weight conditions

$$
I_{\geq 1}|p\rangle^{+}=0, \quad I_{0}|p\rangle^{+}=p|p\rangle^{+} .
$$

Its character is

$$
\chi_{p, k}^{+}(z, q)=\operatorname{Tr}_{\mathcal{H}_{p}^{+}}\left(q^{L_{0}^{+}} z^{I_{0}^{+}}\right)=z^{p} \frac{q^{\frac{1}{2} p\left(p+\sqrt{\frac{2}{k+2}}\right)}}{\prod_{i=1}^{\infty}\left(1-q^{i}\right)} .
$$


Equivalence between $\widehat{s \ell}(2)$ and $N=2$ representations

Lemma III.2 Under the KS mapping, we have the identities

$$
T_{\mathrm{Sug}}+T_{\mathrm{GH}}=T+T^{+}
$$

(where $T$ is the energy-momentum tensor (III.3)), and

$$
J^{0}-B C=-2 H+\frac{k-2}{\sqrt{2(k+2)}} I^{+} .
$$

\section{III.2 The 'anti'-Kazama-Suzuki mapping}

A mapping in the inverse direction to the KS mapping can be defined as follows. It is associated with the 'coset' $\mathcal{A} \otimes u(1) / u(1)$ where the numerator $u(1)$ is represented in terms of the antifermionic system $\psi=e^{\phi}, \psi^{*}=e^{-\phi}$ from section $\amalg$ I.2.2. Then we can identify the affine $s \ell(2)$ generators as

$$
\begin{aligned}
& J^{+}=Q \psi, \quad J^{-}=\frac{3}{3-c} G \psi^{*}, \\
& J^{0}=-\frac{3}{3-c} H+\frac{c}{3-c} \partial \phi .
\end{aligned}
$$

Lemma III.3 For c $\neq 3$, generators (III.12) close to the algebra (ㅍ.2) of the level $k=\frac{2 \mathrm{c}}{3-\mathrm{c}}$, where c is the $N=2$ central charge (the eigenvalue of the central element). Thus, Eqs. (III.12) determine a mapping

$$
F_{\mathrm{KS}}^{-1}: \widehat{s \ell}(2) \rightarrow \mathcal{U} \mathcal{A} \otimes\left[\psi \psi^{*}\right]
$$

The Sugawara energy-momentum tensor ([1.3) takes for the construction (III.12) the form

$$
T^{\mathrm{Sug}}=T+\frac{1}{2} \partial H+\frac{k+2}{4} H H \frac{k+2}{2} H \partial \phi+\frac{k}{4} \partial \phi \partial \phi
$$

We also have a free scalar, with signature -1 , whose modes commute with the $\widehat{s \ell}(2)$ generators (III.12):

$$
I^{-}=\sqrt{\frac{k+2}{2}}(H-\partial \phi) .
$$

The modes $I_{n}^{-}$introduced as $I^{-}(z)=\sum_{n=-\infty}^{\infty} I_{n}^{-} z^{-n-1}$ generate a Heisenberg algebra

$$
\left[I_{n}^{-}, I_{m}^{-}\right]=-n \delta_{m+n, 0} .
$$

This current has the energy-momentum tensor

$$
T^{-}=-\frac{1}{2}\left(I^{-}\right)^{2}-\frac{1}{\sqrt{2(k+2)}} \partial I^{-} .
$$


The module $\mathcal{H}_{q}^{-}$is defined as a Verma module over the Heisenberg algebra (III.16) with the highest-weight vector defined by

$$
I_{n}^{-}|q\rangle^{-}=0, \quad n \geq 1, \quad I_{0}^{-}|q\rangle^{-}=q|q\rangle^{-} .
$$

Lemma III.4 Under the anti-KS mapping we have the identities

$$
T+T_{\phi}=T^{\mathrm{Sug}}+T^{-}
$$

(where $T$ is the energy-momentum tensor of the $N=2$ algebra and $T_{\phi}$ is the energy-momentum tensor of Liouville system ([1.95)), and

$$
-2 H+\partial \phi=J^{0}+\left(\frac{k}{2}-1\right) \sqrt{\frac{k+2}{2}} I^{-} .
$$

\section{III.3 Composing the KS and anti-KS mappings}

Let us consider compositions of the KS and anti-KS mappings.

Lemma III.5 The composition $F_{\mathrm{KS}}^{-1} \circ F_{\mathrm{KS}}$ maps the $\widehat{s \ell}(2)$ algebra into the $\widehat{s \ell}(2)$ algebra in the tensor product $\mathcal{U} \widehat{s \ell}(2) \otimes[B C] \otimes\left[\psi \psi^{*}\right]$ represented by the currents

$$
\begin{aligned}
& \bar{J}^{+}=J^{+} e^{\phi} C, \quad \bar{J}^{-}=J^{-} e^{-\phi} B, \\
& \bar{J}^{0}=J^{0}+\frac{k}{2}(\partial \phi-B C) .
\end{aligned}
$$

For the energy-momentum tensors, we then have

$$
\bar{T}^{\text {Sug }}=T^{\text {Sug }}+\frac{k}{4}\left((\partial \phi)^{2}-2 \partial \phi B C+\partial B C-B \partial C\right)+J^{0}(\partial \phi-B C)
$$

A remarkable feature of these formulas is the appearance of a null current $B C-\partial \phi$; the fact that it is null is crucial for establishing that the generators on the RHSs close to the $\widehat{s \ell}(2)$ algebra.

Remark III.6 The formulas of the lemma can be given a coset-like interpretation by noticing that the image of $\widehat{s \ell}(2)$ under the above embedding is characterized by the fact that it commutes with (the modes of) the currents

$$
\begin{aligned}
I^{+} & =\sqrt{\frac{2}{k+2}}\left(J^{0}+B C\right), \\
F^{-} & =\sqrt{\frac{2}{k+2}}\left(J^{0}-\frac{k}{2} B C+\frac{k+2}{2} \partial \phi\right)
\end{aligned}
$$


Equivalence between $\widehat{s \ell}(2)$ and $N=2$ representations

(these currents do also commute with each other and are matter-like and Liouville-like, respectively).

The same happens with the $N=2$ algebra under the action of $F_{\mathrm{KS}} \circ F_{\mathrm{KS}}^{-1}$ :

Lemma III.7 The composition $F_{\mathrm{KS}} \circ F_{\mathrm{KS}}^{-1}$ maps the $N=2$ algebra into the $N=2$ algebra in the tensor product $\mathcal{U} \mathcal{A} \otimes[B C] \otimes\left[\psi \psi^{*}\right]$ represented by the currents

$$
\begin{aligned}
& \bar{Q}=Q e^{\phi} C, \quad \bar{G}=G e^{-\phi} B, \\
& \bar{H}=H+\frac{k}{k+2}(B C-\partial \phi), \\
& \bar{T}=T+H(B C-\partial \phi)+\frac{k}{2(k+2)}\left((\partial \phi)^{2}-2 \partial \phi B C+\partial^{2} \phi-2 B \partial C\right) .
\end{aligned}
$$

Remark III.8 We also find two currents that commute with the 'target' $N=2$ algebra (III.24):

$$
\begin{aligned}
I^{-} & =\sqrt{\frac{t}{2}}(H-\partial \phi), \\
F^{+} & =\sqrt{\frac{t}{2}}\left(H-\frac{2}{t} B C-\frac{t-2}{t} \partial \phi\right)
\end{aligned}
$$

(these currents are Liouville-like and matter-like respectively, and commute with each other).

\section{III.4 KS and anti-KS mappings at the level of representations}

\section{III.4.1 The Verma module case}

The following theorem is essentially a reformulation of Lemmas ЏII.5 and III.7:

\section{Theorem III.9}

I. The composition of the KS and anti-KS mappings induces an isomorphism of $\widehat{s \ell}(2)$ representations

$$
\mathfrak{M}_{j, k ; \theta} \otimes \Omega \otimes \Xi \approx \bigoplus_{n, m \in \mathbb{Z}} \mathfrak{M}_{j, k ; \theta+n-m} \otimes \mathcal{H}^{+} \sqrt{\frac{2}{k+2}\left(j-\frac{k}{2} \theta-m\right)} \stackrel{\mathcal{H}^{-}}{-\sqrt{\frac{2}{k+2}}\left(j-\frac{k}{2}(\theta-m)-\frac{k+2}{2} n\right)}
$$

where on the LHS the $\widehat{s \ell}(2)$ action is given by the RHSs of (III.21), while on the RHS the $\widehat{s \ell}(2)$ algebra acts naturally on the modules $\mathfrak{M}_{j, k ; \theta}$ and trivially, on the Heisenberg modules.

II. The composition of the anti-KS mapping and the direct KS mapping induces an isomorphism of $N=2$ representations

$$
\mathfrak{V}_{h, t ; \theta} \otimes \Xi \otimes \Omega \approx \bigoplus_{n, m \in \mathbb{Z}} \mathfrak{V}_{h, t ; \theta+m-n} \otimes \mathcal{H}^{-} \sqrt[t]{\frac{t}{2}\left(h-\frac{t-2}{t} \theta+n\right)} \otimes \mathcal{H}_{-\sqrt{\frac{t}{2}\left(h-\frac{t-2}{t}(\theta-n)+\frac{2}{t} m\right)}}^{+}
$$

where on the LHS the $N=2$ algebra acts by generators ([II.24), while on the RHS we have the natural action of $N=2$ on the modules $\mathfrak{V}_{h, t ; \theta}$ and the trivial action on the Heisenberg modules. 
Remark III.10 The theorem remains valid when the $\widehat{s \ell}(2)$ or $N=2$ modules are no longer Verma modules but rather arbitrary modules from the category of highest-weight modules.

In fact, one has a stronger result, which follows from the analysis of each of the mappings $F_{\mathrm{KS}}$ and $F_{\mathrm{KS}}^{-1}$ separately:

\section{Theorem III.11}

I. The KS mapping induces an isomorphism of $N=2$ representations

$$
\mathfrak{M}_{j, k ; \theta} \otimes \Omega \approx \bigoplus_{m \in \mathbb{Z}} \mathfrak{V}_{\frac{-2 j}{k+2}, k+2 ; m-\theta} \otimes \mathcal{H}^{+} \sqrt{\frac{2}{k+2}}\left(j-\frac{k}{2} \theta-m\right)
$$

where on the LHS the $N=2$ algebra acts by generators (III.1) -(III.3), while on the RHS it acts naturally on the twisted topological Verma modules $\mathfrak{V}_{\frac{-2 j}{k+2}, k+2 ; m-\theta}$.

II. The anti-KS mapping induces an isomorphism of $\widehat{s \ell}(2)$ representations

$$
\mathfrak{V}_{h, t ; \theta} \otimes \Xi \approx \bigoplus_{n \in \mathbb{Z}} \mathfrak{M}_{-\frac{t}{2} h, t-2 ; n-\theta} \otimes \mathcal{H}_{\sqrt{\frac{t}{2}}\left(h-\frac{t-2}{t} \theta+n\right)}^{-}
$$

where on the LHS the $\widehat{s \ell}(2)$ algebra acts by generators ([II.12), while on the RHS it acts naturally on the twisted Verma modules $\mathfrak{M}_{-\frac{t}{2} h, t-2 ; n-\theta}$.

Remark III.12 Observe that for $k \in \mathbb{Z}$, each of formulas ([11.28) and ([11.29) translates the periodicity under $\theta \mapsto \theta+2$ on the $\widehat{s \ell}(2)$ side (which is known to hold for integrable representations) into the periodicity under $\theta \mapsto \theta+k+2$ on the $N=2$ side (which, too, is a well-known property of the corresponding $N=2$ representations and conformal models, where it is observed, for example, in the Landau-Ginzburg formulation).

Proof of the Theorem. It is easy to see that the LHS of (III.26) contains each state $|j, k\rangle_{s \ell(2)} \otimes|\theta\rangle_{\mathrm{GH}}$ with all $\theta \in \mathbb{Z}$. Further, we see that each state $|j, k\rangle_{s \ell(2)} \otimes|\theta\rangle_{\mathrm{GH}}$ satisfies the $N=2$ - and Heisenberg annihilation conditions, Eqs. (II.102) and (III.9), respectively. Therefore, each state $|j, k\rangle_{s \ell(2)} \otimes|\theta\rangle_{\mathrm{GH}}$ can be rewritten in the form

$$
|j, k\rangle_{s \ell(2)} \otimes|\theta\rangle_{\mathrm{GH}}=\left|\frac{-2 j}{k+2}, k+2 ; \theta\right\rangle_{\mathrm{top}} \otimes\left|\sqrt{\frac{2}{k+2}}(j-\theta)\right\rangle^{+}, \quad \theta \in \mathbb{Z} .
$$

Thus, as $\theta$ runs over the integers, every $\widehat{s \ell}(2)$ highest-weight state gives rise to infinitely many states satisfying twisted topological highest-weight conditions ([1.108)-([1.109). From any of these states we can generate the twisted topological Verma module $\mathfrak{V}_{\frac{-2 j}{k+2}, k+2 ; \theta}$. All the 
states in every such module have a fixed value of the $I_{0}^{+}$charge (see (III.6)), and this charge is different for modules with different $\theta$. Thus the modules $\mathfrak{V}_{\frac{-2 j}{k+2}, k+2 ; \theta}$ do not overlap for different $\theta \in \mathbb{Z}$. Therefore, we have the mapping

$$
\mathfrak{M}_{j, k ; \theta} \otimes \Omega \longleftarrow \bigoplus_{m \in \mathbb{Z}} \mathfrak{V}_{\frac{-2 j}{k+2}, k+2 ; m-\theta} \otimes \mathcal{H}^{+} \sqrt{\frac{2}{k+2}}\left(j-\frac{k}{2} \theta-m\right)
$$

whose kernel may be different from zero only as a result of the vanishing of some singular vectors in the modules $\mathfrak{V}_{\frac{-2 i}{k+2}, k+2 ; m-\theta}$. In a similar way, we arrive at the mapping

$$
\mathfrak{V}_{h, t ; \theta} \otimes \Xi \longleftarrow \bigoplus_{n \in \mathbb{Z}} \mathfrak{M}_{-\frac{t}{2} h, t-2 ; n-\theta} \otimes \mathcal{H}_{\sqrt{\frac{t}{2}\left(h-\frac{t-2}{t} \theta+n\right)}} .
$$

Now, we tensor ([II.31) with $\Xi$ and make use of (III.32). Note that for the modules under consideration, tensor products preserve exactness. Then the mapping ([11.31) gives rise to

$$
\mathfrak{M}_{j, k ; \theta} \otimes \Omega \otimes \Xi \longleftarrow \bigoplus_{m, n \in \mathbb{Z}} \mathfrak{M}_{j, k ; \theta+n-m} \otimes \mathcal{H}^{+} \sqrt{\frac{2}{k+2}\left(j-\frac{k}{2} \theta-m\right)} \stackrel{\mathcal{H}^{-}}{-\sqrt{\frac{2}{k+2}}\left(j-\frac{k}{2}(\theta-m)-\frac{k+2}{2} n\right)},
$$

which is an isomorphism by Theorem [II.9, hence both (III.31) and (III.32) are isomorphisms.

Corollary 1. Singular vectors in the $\widehat{s \ell}(2)$ Verma modules and in the topological $N=2$ Verma modules occur (or do not occur) simultaneously. Thus formulas (II.113) for positions of the topological singular vectors are re-derived by translating the positions of the $\widehat{s \ell}(2)$ singular vectors under the KS mapping.

Corollary 2. Let there exist a singular vector with the $J_{0}^{0}$-charge equal to $r$ in the $\widehat{s \ell}(2)$ Verma module on the LHS of ([II.31); then each topological $N=2$ Verma module $\mathfrak{V}_{\frac{-2 j}{k+2}, k+2 ; m-\theta}$ from the RHS of (III.31) contains a topological singular vector, which satisfies highest-weight conditions (II.108) with $\theta \rightarrow m-\theta+r$.

Proof Let us consider the tensor product $|S(r)\rangle \otimes|\lambda\rangle_{\mathrm{GH}}$, where $|S(r)\rangle$ denotes a singular vector with the charge $r$ in the $\widehat{s \ell}(2)$ Verma module from the LHS of (III.31) and $|\lambda\rangle_{\mathrm{GH}}$ is the

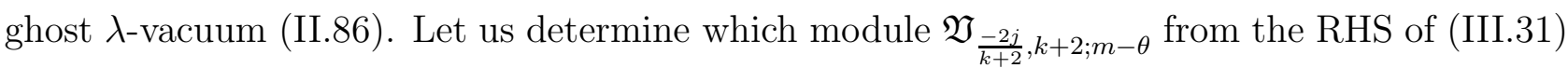
the state $|S(r)\rangle \otimes|\lambda\rangle_{\mathrm{GH}}$ belongs to. To this end, we calculate the eigenvalue of $I_{0}^{+}$(see (III.6)) on the state $|S(r)\rangle \otimes|\lambda\rangle_{\mathrm{GH}}$ :

$$
I_{0}^{+}|S(r)\rangle \otimes|\lambda\rangle_{\mathrm{GH}}=\sqrt{\frac{2}{k+2}}\left(j+r-\frac{k}{2}-\lambda\right)|S(r)\rangle \otimes|\lambda\rangle_{\mathrm{GH}} .
$$


Therefore, the state $|S(r)\rangle \otimes|m+r\rangle_{\mathrm{GH}}$ belongs to $\mathfrak{V}_{\frac{-2 i}{k+2}, k+2 ; m-\theta}$ and, as follows easily from formulas (ㅍ.86) and (ㅍ․1), satisfies highest-weight conditions (ㅍ.108) with $\theta \rightarrow m-\theta+r$.

A constructive description of the correspondence between singular vectors is considered in subsection IV.1.

Corollary 3. The isomorphism established in the Theorem implies an identity for the characters:

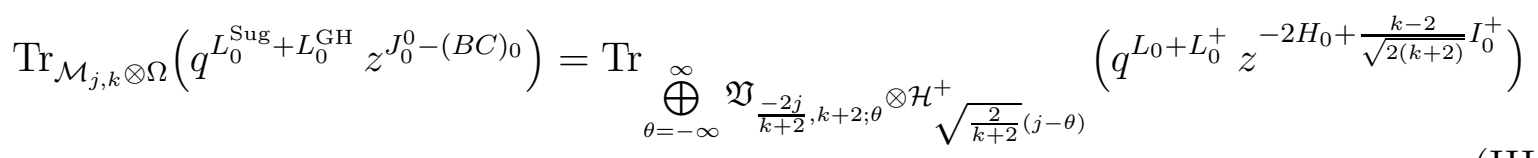

Inserting the characters, given above, of each of the modules involved, this can be rewritten as the following formal identity:

$$
\begin{aligned}
& \frac{\prod_{i=0}^{\infty}\left(1+z q^{i}\right) \prod_{i=1}^{\infty}\left(1+z^{-1} q^{i}\right)}{\prod_{i=0}^{\infty}\left(1-z^{-1} q^{i}\right) \prod_{i=1}^{\infty}\left(1-z q^{i}\right)} \\
& \quad=\frac{\prod_{i=1}^{\infty}\left(1+z^{2} q^{i}\right) \prod_{i=1}^{\infty}\left(1+z^{-2} q^{i}\right)}{\prod_{i=1}^{\infty}\left(1-q^{i}\right)^{2}}\left(1+\left(1+z^{2}\right) \sum_{r=1}^{\infty}\left[\frac{z^{-r}}{1+z^{2} q^{r}}+\frac{q^{r} z^{r-2}}{1+z^{-2} q^{r}}\right]\right) .
\end{aligned}
$$

\section{III.4.2 The relaxed/massive Verma module case}

Now, we give an extension of theorems [II.9 [II.11 to the relaxed and massive Verma modules.

\section{Theorem III.13}

I. The composition of the $K S$ and anti-KS mappings induces an isomorphism of $\widehat{s \ell}(2)$ representations

$$
\mathfrak{R}_{j, \Lambda, k ; \theta} \otimes \Omega \otimes \Xi \approx \bigoplus_{n, m \in \mathbb{Z}} \mathfrak{R}_{j, \Lambda, k ; \theta+n-m} \otimes \mathcal{H}_{\sqrt{\frac{2}{k+2}}\left(j-\frac{k}{2} \theta-m\right)} \otimes \mathcal{H}_{-\sqrt{\frac{2}{k+2}}\left(j-\frac{k}{2}(\theta-m)-\frac{k+2}{2} n\right)}
$$

where on the LHS the $\widehat{s \ell}(2)$ action is given by the RHSs of (III.21), while on the RHS the $\widehat{s \ell}(2)$ algebra acts naturally on the modules $\mathfrak{R}_{j, \Lambda, k ; \theta}$ and trivially, on the Heisenberg modules.

II. The composition of the anti-KS mapping and the direct KS mapping induces an isomor- 
Equivalence between $\widehat{s \ell}(2)$ and $N=2$ representations

phism of $N=2$ representations

$$
\mathfrak{W}_{h, \ell, t ; \theta} \otimes \Xi \otimes \Omega \approx \bigoplus_{n, m \in \mathbb{Z}} \mathfrak{W}_{h, t, \ell ; \theta+m-n} \otimes \mathcal{H}_{\sqrt{\frac{t}{2}\left(h-\frac{t-2}{t} \theta+n\right)}}^{-} \otimes \mathcal{H}_{-\sqrt{\frac{t}{2}\left(h-\frac{t-2}{t}(\theta-n)+\frac{2}{t} m\right)}}^{+}
$$

where on the LHS the $N=2$ algebra acts by generators (III.24), while on the RHS we have the natural action of $N=2$ on the modules $\mathfrak{W}_{h, \ell, t ; \theta}$ and the trivial action on the Heisenberg modules.

This allows us to obtain, similarly to the above,

\section{Theorem III.14}

I. The KS mapping induces an isomorphism of $N=2$ representations

$$
\mathfrak{R}_{j, \Lambda, k ; \theta} \otimes \Omega \approx \bigoplus_{\lambda \in \mathbb{Z}} \mathfrak{U}_{\frac{-2 j}{k+2}, \frac{\Lambda}{k+2}, k+2 ; \lambda-\theta} \otimes \mathcal{H}^{+} \sqrt{\frac{2}{k+2}}\left(j-\frac{k}{2} \theta-\lambda\right)
$$

where on the LHS the $N=2$ algebra acts by generators (III.1)-(III.3), while on the RHS it acts naturally on the twisted massive Verma modules $\mathfrak{U}_{\frac{-2 j}{k+2}, \ell, k+2 ; \lambda-\theta}$.

II. The anti-KS mapping induces an isomorphism of $\widehat{s \ell}(2)$ representations

$$
\mathfrak{U}_{h, \ell, t ; \theta} \otimes \Xi \approx \bigoplus_{n \in \mathbb{Z}} \mathfrak{R}_{-\frac{t}{2} h, t \ell, t-2 ; n-\theta} \otimes \mathcal{H}_{\sqrt{\frac{t}{2}}\left(h-\frac{t-2}{t} \theta+n\right)}^{-}
$$

where on the LHS the $\widehat{s \ell}(2)$ algebra acts by generators (III.12), while on the RHS it acts naturally on the twisted relaxed Verma modules $\mathfrak{R}_{-\frac{t}{2} h, t \ell, t-2 ; n-\theta}$. 
We could word by word repeat the proof of theorems [II.9 III.11 in the case of relaxed Verma modules. Instead, we illustrate the main points by the following diagram:

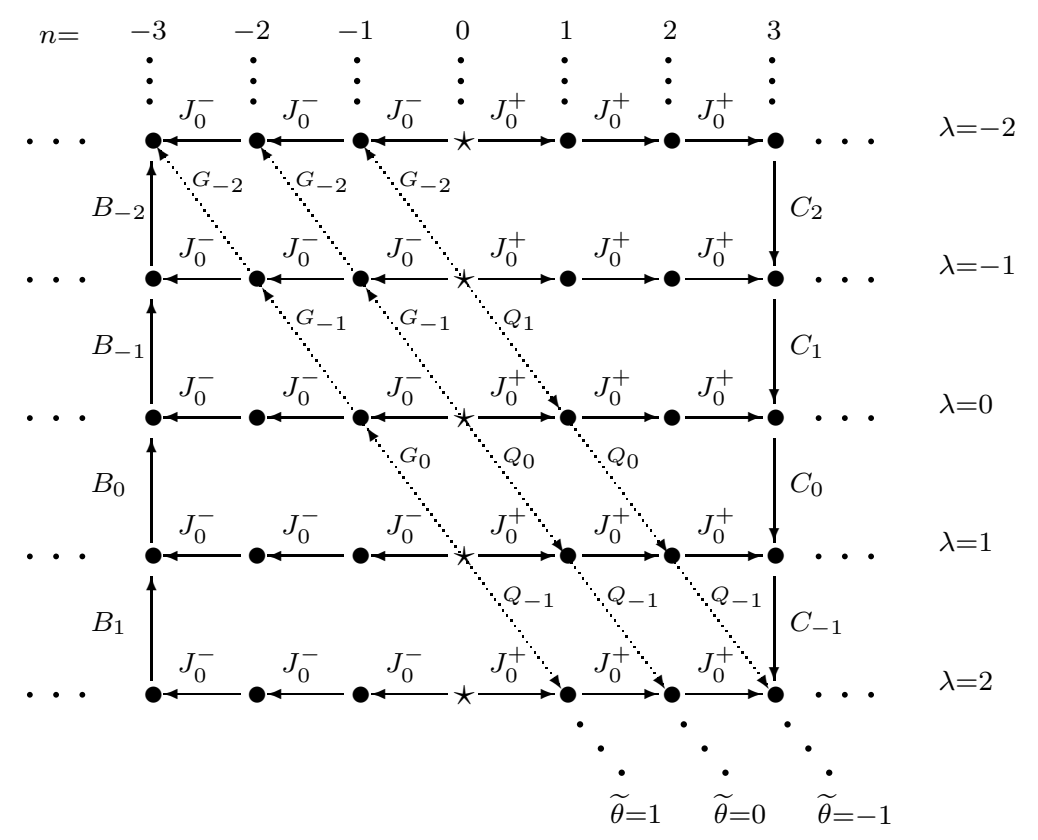

This describes the case of $\theta=0$ in (III.38) (which is the $\widehat{s \ell}(2)$ 'spectral' parameter, not to be confused with the one labelling twisted $N=2$ modules that are also present in the diagram; the $N=2$ spectral parameter is denoted by $\widetilde{\theta}$ here). The diagram represents the tensor product of the relaxed Verma module extremal diagram (11.18) with a ghost extremal diagram. The latter consists of ghost vacua ([I.86) taken in different pictures. As we have chosen $\theta=0$, the $\widehat{s \ell}(2)$ arrows are horizontal, as in (11.18), while the ghost ones are shown vertical. For simplicity, the ghost $(B$ and $C$ ) arrows are shown explicitly only in two columns. Since the different pictures (11.86) in the free-fermion system are all equivalent, all the vertical arrows are invertible, however we have separated the $B$ and $C$ arrows in the diagram, trying to keep it readable. Thus the $\bullet$ dots denote $|j, \Lambda, k| n\rangle_{s \ell(2)} \otimes|\lambda\rangle_{\mathrm{GH}}$, the values of $n$ being written in the upper row and those of $\lambda$, in the right column. The $\star$ s denote $|j, \Lambda, k\rangle_{s \ell(2)} \otimes|\lambda\rangle_{\mathrm{GH}}$. Now, the dotted lines are precisely the extremal diagrams ([I.121) of massive Verma modules $\mathfrak{U}_{h, \ell, t ; \tilde{\theta}}$ from the RHS of ([11.38), viewed from above (the grading with respect to the level is in the direction orthogonal to the page).

Note also that the analogues of corollaries 1 and 2 from the previous subsection are straightforward to formulate in the relaxed/massive case. 


\section{Equivalence between categories}

\section{IV.1 Equivalence of Verma chain categories}

It follows from the above analysis that an equivalence between some categories of $\widehat{s \ell}(2)$ and $N=2$ modules can only be established for those categories which effectively allow for a factorization with respect to the spectral flow. These can be defined as follows.

Let us consider the objects that are infinite chains $\left(\mathfrak{M}_{j, k ; \theta}\right)_{\theta \in \mathbb{Z}}$, where $\mathfrak{M}_{j, k ; \theta}$ are twisted Verma modules. As a morphisms between $\left(\mathfrak{M}_{j, k ; \theta}\right)_{\theta \in \mathbb{Z}}$ and $\left(\mathfrak{M}_{j^{\prime}, k^{\prime} ; \theta}\right)_{\theta \in \mathbb{Z}}$, we take any Verma module morphisms $\mathfrak{M}_{j, k ; \theta_{1}} \rightarrow \mathfrak{M}_{j^{\prime}, k^{\prime} ; \theta_{2}}$. Let us call this category the $\widehat{s \ell}(2)$ Verma chain category $\mathcal{C} \mathcal{V} \mathcal{R}$.

On the $N=2$ side, the topological Verma chain category $\mathcal{C} \mathcal{T} \mathcal{V} \mathcal{E}$ is defined similarly. Namely, one takes the chains of twisted topological Verma modules, every such chain comprising the modules that are the spectral flow transforms of a topological Verma module with all $\theta \in \mathbb{Z}$. Morphisms of the chains are defined similarly to the $\widehat{s l}(2)$ case. $]^{8}$

The meaning of the definition of morphisms of chains is that, obviously, given a morphism between any two modules, one spreads it over the entire chains by spectral flow transforms.

To define a functor relating such chains, we first construct correspondences between individual modules in the chains:

Given a topological Verma module $\mathfrak{V}_{h, t ; \theta}$, and an arbitrary $\theta^{\prime} \in \mathbb{Z}$, take the Heisenberg modules $\mathcal{H}_{-\sqrt{\frac{2}{t}}\left(\frac{t}{2} j+\frac{t}{2} \theta^{\prime}-\theta\right)}$ and consider

$$
\mathfrak{V}_{h, t ; \theta} \otimes \mathcal{H}_{-\sqrt{\frac{2}{t}}\left(\frac{t}{2} j+\frac{t}{2} \theta^{\prime}-\theta\right)} \oplus \bigoplus_{m \in \mathbb{Z}, m \neq 0} \mathfrak{V}_{h, t ; \theta+m} \otimes \mathcal{H}_{-\sqrt{\frac{2}{t}}\left(\frac{t}{2} j+\frac{t}{2} \theta^{\prime}-\theta+m\right)}^{+}
$$

where $\mathfrak{V}_{h, t ; \theta+m}$ are the images of $\mathfrak{V}_{h, t ; \theta}$ under the spectral flow. By theorem [II.9, (IV.1) is isomorphic to the tensor product of an $\widehat{s \ell}(2)$ Verma module $\mathfrak{M}_{-\frac{t}{2} h, t-2 ; \theta^{\prime}}$ with a ghost module. We define the result of applying $F_{\mathrm{KS}}\left(\theta, \theta^{\prime}\right)$ to $\mathfrak{V}_{h, t ; \theta}$ to be the module $\mathfrak{M}_{-\frac{t}{2} h, t-2 ; \theta^{\prime}}$ :

$$
F_{\mathrm{KS}}\left(\theta, \theta^{\prime}\right): \mathfrak{V}_{h, t ; \theta} \leadsto \mathfrak{M}_{-\frac{t}{2} h, t-2 ; \theta^{\prime}}, \quad \theta, \theta^{\prime} \in \mathbb{Z}
$$

A similar definition can be given for the correspondence

$$
F_{\mathrm{KS}}^{-1}\left(\theta, \theta^{\prime}\right): \mathfrak{M}_{j, k ; \theta} \leadsto \mathfrak{V}_{-\frac{2}{k+2} j, k+2 ; \theta^{\prime}}, \quad \theta, \theta^{\prime} \in \mathbb{Z}
$$

\footnotetext{
${ }^{8}$ It is important to note here that any submodule of a (twisted) topological Verma module is a twisted topological Verma module.
} 
Given a Verma module $\mathfrak{M}_{j, k ; \theta}$ and $\theta^{\prime} \in \mathbb{Z}$, we construct the sum

$$
\mathfrak{M}_{j, k ; \theta} \otimes \mathcal{H}_{-\sqrt{\frac{2}{k+2}}\left(j+\theta^{\prime}-\frac{k+2}{2} \theta\right)}^{\oplus} \bigoplus_{n \in \mathbb{Z}, n \neq 0} \mathfrak{M}_{j+\frac{k}{2} n, k ; \theta+n} \otimes \mathcal{H}_{-\sqrt{\frac{2}{k+2}}\left(j+\theta^{\prime}-\frac{k+2}{2} \theta+n\right)}
$$

which, by theorem 【II.9, is isomorphic to the module $\mathfrak{V}_{-\frac{2}{k+2} j, k+2 ; \theta^{\prime}}$ tensored with a module of antifermions. This twisted topological $N=2$ module is by definition the result of applying $F_{\mathrm{KS}}^{-1}\left(\theta, \theta^{\prime}\right)$ to $\mathfrak{M}_{j, k ; \theta}$.

While the correspondences from (IV.2) and (IV.3) depend on the chosen $\theta$ and $\theta^{\prime}$, the $\theta$ dependence disappears when applied to the elements of $\mathcal{C} \mathcal{E} \mathcal{R}$ and $\mathcal{C} \mathcal{T V E R}$; therefore $F_{\mathrm{KS}}(\cdot, \cdot)$ and $F_{\mathrm{KS}}^{-1}(\cdot, \cdot)$, which we denote again by $F_{\mathrm{KS}}$ and $F_{\mathrm{KS}}^{-1}$, are candidates for the functors

$$
\begin{aligned}
& F_{\mathrm{KS}}: \mathcal{C} \mathcal{T V E R} \leadsto \mathcal{C} \mathcal{V} \mathcal{R} \\
& F_{\mathrm{KS}}^{-1}: \mathcal{C V E R} \leadsto \mathcal{C} \mathcal{T} \mathcal{V} \mathcal{R}
\end{aligned}
$$

Evidently, the composition of $F_{\mathrm{KS}}$ and $F_{\mathrm{KS}}^{-1}$ maps each chain of twisted Verma modules into an isomorphic chain. Therefore, $F_{\mathrm{KS}}$ and $F_{\mathrm{KS}}^{-1}$ would be the direct and inverse functors, thus establishing the isomorphism of categories, once we define how $F_{\mathrm{KS}}$ and $F_{\mathrm{KS}}^{-1}$ act on morphisms.

Recall that in Verma-module categories, morphisms are naturally identified with singular vectors. As we see from the isomorphisms of section 피.4, an $\widehat{s \ell}(2)$ singular vector exists in a twisted Verma module $\mathfrak{M}_{j, k ; \theta}$ (hence in all those with $\theta \mapsto \theta+n, n \in \mathbb{Z}$ ) if and only if a topological singular vector exists in at least one (hence in each) twisted topological Verma module $\mathfrak{V}_{\frac{-2 j}{k+2}, k+2 ; m}, m \in \mathbb{Z}$, from the RHS of ([II.28). The following lemma gives an explicit mapping between the 'building blocks' of the corresponding singular vectors:

Lemma IV.1 The KS mapping induces a correspondence between the 'continued' objects, $\left(J_{-\theta}^{-}\right)^{\nu-\mu+1}$ and $\left(J_{\theta-1}^{+}\right)^{\nu-\mu+1}$ on the one hand, and $g(\mu, \nu), q(\mu, \nu)$ on the other hand, which act on the respective highest weights as shown in (II.11) and (II.112). The correspondence reads

$$
\begin{array}{ll}
F_{\mathrm{KS}}\left(\theta^{\prime}, \theta\right): g(\mu, \nu) \mapsto\left(J_{-\theta}^{-}\right)^{\nu-\mu+1} b(\mu, \nu), & F_{\mathrm{KS}}\left(\theta^{\prime}, \theta\right): q(\mu, \nu) \mapsto\left(J_{\theta-1}^{+}\right)^{\nu-\mu+1} c(\mu, \nu), \\
F_{\mathrm{KS}}^{-1}\left(\theta, \theta^{\prime}\right):\left(J_{-\theta}^{-}\right)^{\nu-\mu+1} \mapsto g(\mu, \nu) e^{-(\nu-\mu+1) \phi}, & F_{\mathrm{KS}}^{-1}\left(\theta, \theta^{\prime}\right):\left(J_{\theta-1}^{+}\right)^{\nu-\mu+1} \mapsto q(\mu, \nu) e^{(\nu-\mu+1) \phi .} .
\end{array}
$$

This leads us to

Theorem IV.2 The KS and anti-KS mappings give rise to identifications, which we denote again by $F_{\mathrm{KS}}\left(\theta, \theta^{\prime}\right)$ and $F_{\mathrm{KS}}^{-1}\left(\theta, \theta^{\prime}\right)$ respectively, between the twisted $\widehat{s \ell}(2)$ singular vectors (II.14) 
in the Verma module $\mathfrak{M}_{j, k ; \theta}$ and the $N=2$ singular vectors (【I.114) and (【I.115) $|E(r, s, t)\rangle^{ \pm, \theta}$ in the twisted topological Verma modules $\mathfrak{V}_{-\frac{2}{k+2} j, k+2 ; \theta}$ :

$$
\begin{aligned}
& F_{\mathrm{KS}}\left(\theta, \theta^{\prime}\right):|E(r, s, k+2)\rangle^{ \pm, \theta} \mapsto\left|S_{ \pm}^{\mathrm{MFF}}(r, s, k)\right\rangle^{\theta^{\prime}} \\
& F_{\mathrm{KS}}^{-1}\left(\theta, \theta^{\prime}\right):\left|S_{ \pm}^{\mathrm{MFF}}(r, s, k)\right\rangle^{\theta} \mapsto|E(r, s, k+2)\rangle^{ \pm, \theta^{\prime}},
\end{aligned}
$$

where $|E(r, s, t)\rangle^{ \pm, \theta}$ and $\left|S_{ \pm}^{\mathrm{MFF}}(r, s, k)\right\rangle^{\theta^{\prime}}$ denote the singular vectors transformed by the corresponding spectral flows.

Evidently, $F_{\mathrm{KS}}(\cdot, \cdot)$ and $F_{\mathrm{KS}}^{-1}(\cdot, \cdot)$ applied to the chains of the respective Verma modules take morphisms (between chains) into morphisms. Thus we have made $F_{\mathrm{KS}}$ and $F_{\mathrm{KS}}^{-1}$ into functors. The results of this subsection can be summarized in the following theorem:

Theorem IV.3 The functors $F_{\mathrm{KS}}$ and $F_{\mathrm{KS}}^{-1}$ are covariant functors which are inverse to each other and which therefore establish the equivalence between the Verma chain category $\mathcal{C} \mathcal{V} \mathcal{R}$ on the $\widehat{s \ell}(2)$ side and the topological Verma chain category $\mathcal{C} \mathcal{T} \mathcal{V E R}$ on the $N=2$ side.

\section{IV.2 Extending the equivalence to highest-weight-type categories}

We have seen in the previous subsection that the $\widehat{s \ell}(2)$ Verma, and $N=2$ topological Verma chain categories are equivalent. Now we would like to extend the equivalence to larger categories. Let us define the highest-weight type chain category:

Definition IV.4 The highest-weight type chain category $\mathcal{C H W}$ is the category whose objects are chains of modules from the category $\mathcal{H} \mathcal{W}$ of $\widehat{s \ell}(2)$ highest-weight type representations twisted by the spectral flow with integer parameters. A morphism between two such chains $\left(\mathcal{N}_{\theta}\right)_{\theta \in \mathbb{Z}}$ and $\left(\mathcal{N}_{\theta}^{\prime}\right)_{\theta \in \mathbb{Z}}$ is any morphism $\mathcal{N}_{\theta_{1}} \rightarrow \mathcal{N}_{\theta_{2}}^{\prime}$ (which is understood to be spread over the entire chains by the spectral flow).

Remark IV.5 As an aside, note that the condition for any such chain to admit non-trivial automorphisms singles out the minimal $s \ell(2)$ models, and the same is true for the chains of $N=2$ modules. Then, taking the factors with respect to the respective spectral flows, we would have the same number of fields in the $s \ell(2)$ and $N=2$ minimal models. Thus, the minimal models of these algebras can be thought of as periodical chains of representations; we hope to demonstrate elsewhere the usefulness of such a viewpoint. 
On the $N=2$ side, these twisted categories mix into one category because submodules of a (twisted) topological Verma module are the twisted topological Verma modules with different $\theta$.

Definition IV.6 The topological chain category $\mathcal{C} \mathcal{T O P}$ is the category whose objects are chains of modules from the topological category $\mathcal{T O P}$ twisted by the spectral flow with integer parameters. A morphism between two such chains $\left(\mathcal{U}_{\theta}\right)_{\theta \in \mathbb{Z}}$ and $\left(\mathcal{U}_{\theta}^{\prime}\right)_{\theta \in \mathbb{Z}}$ is any morphism $\mathcal{U}_{\theta_{1}} \rightarrow \mathcal{U}_{\theta_{2}}^{\prime}$ of $N=2$ modules.

Theorem IV.7 The functors $F_{\mathrm{KS}}$ and $F_{\mathrm{KS}}^{-1}$ establish the equivalence between categories $\mathcal{C} \mathcal{H}$ and $\mathcal{C} \mathcal{T O P}$.

\section{IV.3 Equivalence of the relaxed and massive Verma chain categories}

We define chains of relaxed Verma modules on the $\widehat{s \ell}(2)$ side and chains of massive Verma modules on the $N=2$ side as this was done in Sec. IV.1 Then, we formulate the theorem, whose proof is a consequence of Lemma IV.1:

Theorem IV.8 The $K S$ and anti-KS mappings gives rise to identifications, which we denote again by $F_{\mathrm{KS}}\left(\theta, \theta^{\prime}\right)$ and $F_{\mathrm{KS}}^{-1}\left(\theta, \theta^{\prime}\right)$ respectively, between

1) singular vectors (II.45) and (ㅍ.46) in the relaxed Verma module $\mathfrak{R}_{j, \Lambda, k ; \theta}$ and $N=2$ singular vectors ([1.129), $|S(r, s, h, t)\rangle^{\mp, \theta}$, in the twisted massive Verma modules $\mathfrak{U}_{-\frac{2}{k+2} j, \frac{\Lambda}{k+2}, k+2 ; \theta}$ :

$$
\begin{aligned}
& F_{\mathrm{KS}}\left(\theta, \theta^{\prime}\right):\left|\Sigma^{ \pm}(r, s, j, k)\right\rangle^{\theta} \mapsto\left|S\left(r, s, \frac{-2 j}{k+2}, k+2\right)\right\rangle^{\mp, \theta^{\prime}}, \\
& F_{\mathrm{KS}}^{-1}\left(\theta, \theta^{\prime}\right):|S(r, s, h, t)\rangle^{\mp, \theta} \mapsto\left|\Sigma^{ \pm}\left(r, s,-\frac{t}{2} h, t-2\right)\right\rangle^{\theta^{\prime}},
\end{aligned}
$$

and also between

2) charged $\widehat{s \ell}(2)$ singular vectors ([I.30) and charged $N=2$ singular vectors ([I.126):

$$
\begin{aligned}
& F_{\mathrm{KS}}\left(\theta, \theta^{\prime}\right):|C(r, j, k)\rangle^{\theta} \mapsto|E(r, h, t)\rangle_{\mathrm{ch}}^{\theta^{\prime}}, \\
& F_{\mathrm{KS}}^{-1}\left(\theta, \theta^{\prime}\right):|E(r, h, t)\rangle_{\mathrm{ch}}^{\theta} \mapsto|C(r, j, k)\rangle^{\theta^{\prime}},
\end{aligned}
$$

where $\left|\Sigma^{ \pm}(r, s, j, k)\right\rangle^{\theta}$ and $|S(r, s, h, t)\rangle^{\mp, \theta^{\prime}}$ denote the respective singular vectors transformed by the corresponding spectral flows. 
Again, as in Sec. IV.1, we can formulate

Theorem IV.9 The functors $F_{\mathrm{KS}}$ and $F_{\mathrm{KS}}^{-1}$ are covariant functors which are inverse to each other and which therefore establish the equivalence of the relaxed Verma chain category $\mathcal{C} \mathcal{R} \mathcal{V} \mathcal{R}$ on the $\widehat{s \ell}(2)$ side and the massive Verma chain category $\mathcal{C M V E R}$ on the $N=2$ side.

\section{IV.4 Extending the equivalence to the relaxed highest-weight-type categories}

We now formulate an extension of the categorial equivalence $F_{\mathrm{KS}}$ and $F_{\mathrm{KS}}^{-1}$ to the case of the largest categories $\mathcal{C R \mathcal { W }}$ and $\mathcal{C} \mathcal{M H W}$ in diagram (I.1). The definitions of the chain categories $\mathcal{C R H W}$ and $\mathcal{C M H \mathcal { W }}$ can be obtained by means of a step-by-step repetition of Definitions IV.4 and $1 \nabla .6$ and are omitted. Then the analog of Theorem $\mathbb{T V . 7}$ reads

Theorem IV.10 The functors $F_{\mathrm{KS}}$ and $F_{\mathrm{KS}}^{-1}$ establish the equivalence between categories $\mathcal{C R H \mathcal { W }}$ and $\mathcal{C M H \mathcal { W }}$.

\section{Concluding remarks}

We believe that the issues addressed in this paper will be essential in a number of problems in the $\widehat{s \ell}(2)$ and $N=2$ representation theories as well as in conformal field theory.

Comparing the $\widehat{s \ell}(2)$ and $N=2$ highest-weight representations, it may be observed that the occurrence of infinitely many highest-weight states in $\widehat{s \ell}(2)$ relaxed Verma modules is intuitively more 'obvious' than in its $N=2$ counterpart; all of the $\widehat{s \ell}(2)$ relaxed-highest-weight states are on the same floor in the extremal diagram, whereas in the $N=2$ case this is not so: extremal diagrams of untwisted massive $N=2$ modules contain only two vectors at the top level, and it may be tempting to assign to these a more fundamental status than to the other vectors from the extremal diagram. Working with the top-level representative of $N=2$ extremal diagrams conceals the 'topological' nature of some of submodules in massive $N=2$ Verma modules (see [14] for a detailed description of the structure of $N=2$ Verma modules).

As to the structure of relaxed- $\widehat{s \ell}(2)$ or massive $N=2$ Verma modules, we have seen that a significant difference from the familiar Virasoro or the usual- $\widehat{s \ell}(2)$ case is that (to use the $N=2$ language) a given massive $N=2$ Verma module may contain submodules of two types, the twisted topological and the massive ones. An important point is that every submodule can 
be freely generated from a twisted (topological) highest-weight vector (singular vectors (II.114) and (II.115) in the topological Verma modules and ([1.126) and (II.129) in massive Verma modules). From these results, it is not too difficult to derive the complete classification of embedding diagrams of the relaxed- $\widehat{s \ell}(2)$ and massive- $N=2$ Verma modules [26]. These follow (an extension of) the familiar I-II-III pattern; the embedding structure can be described without invoking subsingular vectors [14], since the above singular vectors generate maximal submodules (that subsingular vectors in massive- $N=2$ /relaxed- $\widehat{s \ell}(2)$ modules are superfluous can most clearly be seen in the $\widehat{s \ell}(2)$ language).

Another problem is the derivation of fusion rules. In order to completely define the fusion rules one has to fix, among other things, the category of representations. On the $\widehat{s \ell}(2)$ side, the fusion rules have been obtained in [1, 2, 4]. However, these do not include the twisted (spectral-flow-transformed) $\widehat{s \ell}(2)$ representations, whose contribution is to be expected on general grounds [2]. This is related to the fact that only the quantum group $\operatorname{osp}(1 \mid 2)_{q}$ has so far been observed as a symmetry of $\widehat{s \ell}(2)$ fusion rules, while a larger quantum group $s \ell(2 \mid 1)_{q}$ is to be expected. This is an indication that some modules are still missing from the known fusion; we hope that we have described the representation-theoretic part of the desired more general construction. Then the complete $\widehat{s \ell}(2)$ fusion rules factored with respect to the spectral flow would be isomorphic to complete $N=2$ fusion rules factored over the $N=2$ spectral flow.

Acknowledgements. We wish to thank F. Malikov and V. Sirota for useful remarks. AMS is also grateful to C. Preitschopf for discussions. The work of AMS and IYT was supported in part by RFFI Grant 96-01-00725, the work of IYT was supported in part by a Landau Foundation grant, and the work of AMS, by grant \#93-0633-ext from the European Community.

\section{References}

[1] H. Awata and Y. Yamada, Fusion Rules for the Fractional Level sl(2) Algebra, Mod. Phys. Lett. A7, 1185 (1992).

[2] B. Feigin and F. Malikov, Modular Functor and Representation Theory of $\widehat{s l}_{2}$, Cont. Math. 202 "Operads: Proceedings of Reneissance Conferences".

[3] M. Ademollo, L. Brink, A. D’Adda, R. D’Auria, E. Napolitano, S. Sciuto, E. Del Guidice, P. Di Vecchia, S. Ferrara, F. Gliozzi, R. Musto, and R. Pettorino, Dual String With U(1) Color Symmetry, Phys. Lett. B62, 105 (1976);

M. Ademollo, L. Brink, A. D'Adda, R. D'Auria, E. Napolitano, S. Sciuto, E. Del Guidice, 
P. Di Vecchia, S. Ferrara, F. Gliozzi, R. Musto, R. Pettorino, and J.H. Schwarz, Dual String Models With Nonabelian Color and Flavor Symmetries, Nucl. Phys. B111, 77 (1976).

[4] O. Andreev, Operator Algebra of the SL(2) Conformal Field Theories, Phys. Lett. B363, 166 (1995).

[5] J.L. Petersen, J. Rasmussen, and M. Yu, Fusion, Crossing and Monodromy in Conformal Field Theory Based on SL(2) Current Algebra with Fractional Level, Nucl. Phys. B481, 577-624 (1996).

[6] P. Furlan, A.Ch. Ganchev, and V.B. Petkova, $A_{1}^{(1)}$ admissible representations-fusion transformations and local correlators, Nucl. Phys. B491, 635 (1997).

[7] A.M. Semikhatov, The MFF singular vectors in topological conformal theories, Mod. Phys. Lett. A9, 1867 (1994).

[8] V.G. Kač Infinite Dimensional Lie Algebras, Cambridge University Press 1990.

[9] A.M. Semikhatov and I.Yu. Tipunin, Singular Vectors of the Topological Conformal Algebra, Int. J. Mod. Phys. A11, 4597 (1996).

[10] A.M. Semikhatov and I.Yu. Tipunin, All Singular Vectors of the $N=2$ Superconformal Algebra via the Algebraic Continuation Approach, hep-th/9604176.

[11] K. Bardakçi and M.B. Halpern, Phys. Rev. D3, 2493 (1971).

[12] W. Boucher, D. Friedan, and A. Kent, Determinant formulae and Unitarity for the N=2 Superconformal Algebras in Two-Dimensions Or Exact Results on String Compactification, Phys. Lett. B172, 316 (1986).

[13] F.G. Malikov, B.L. Feigin, and D.B. Fuchs, Singular Vectors in Verma Modules over Kač-Moody Algebras, Funk. An. Prilozh. 20 N2, 25 (1986).

[14] A.M. Semikhatov and I.Yu. Tipunin, The Structure of Verma Modules over the $N=2$ Superconformal Algebra, hep-th/9704111. Commun. Math. Phys., to appear.

[15] A. Schwimmer and N. Seiberg, Comments on the $N=2, N=3, N=4$ Superconformal Algebras in Two-Dimensions, Phys. Lett. B184 (1987) 191.

[16] W. Lerche, C. Vafa, and N.P. Warner, Chiral Rings in $N=2$ Superconformal Theories, Nucl. Phys. B324, 427 (1989).

[17] B.L. Feigin and A.V. Stoianovsky Functional Models of Representations of Current Algebras and Semi-infinite Schubert Cells, Funk. An. i ego prilozh., 28(1), 68 (1994).

[18] A.M. Semikhatov, Verma Modules, Extremal Vectors, and Singular Vectors on the Non-Critical $N=2$ String Worldsheet, hep-th/9610084.

[19] P. Di Vechia, J.L. Petersen, M. Yu, and H.B. Zheng, Phys. Lett. B174, 280 (1986).

[20] Y. Kazama and H. Suzuki, Nucl. Phys. B321, 232 (1989).

[21] V.G. Kač and D.A. Kazhdan, Structure of Representations with Highest Weight of InfiniteDimensional Lie Algebras, Adv. Math. 34, 97 (1979).

[22] F. Malikov, Algebra i Analiz, 2 No. 2, 65 (1990).

[23] D.H. Friedan, E.J. Martinec, and S.H. Shenker, Conformal Invariance, Supersymmetry and String Theory, Nucl. Phys. B271, 93 (1986).

[EHY] T. Eguchi, S. Hosono, and S.-K. Yang, Hidden Fermionic Symmetry in Conformal Topological Field Theories, Commun. Math. Phys. 140, 159 (1991).

$[24]$ B. Gato-Rivera and A.M. Semikhatov, $d \leq 1 \cup d \geq 25$ and $W$ Constraints From BRST-Invariance in the $c \neq 3$ Topological Algebra, Phys. Lett. B293, 72 (1992).

[25] M. Bershadsky, W. Lerche, D. Nemeschansky, and N.P. Warner, Extended $N=2$ Superconformal Structure of Gravity and W Gravity Coupled to Matter, Nucl. Phys. B401, 304-347 (1993). 
[26] A.M. Semikhatov and V.A. Sirota, Embedding Diagrams of $N=2$ and Relaxed-ŝl(2) Verma Modules, hep-th/9712102. 